\title{
Friction and diffusive light transport in a granular medium
}

\author{
Dissertation \\ zur Erlangung des mathematisch-naturwissenschaftlichen \\ Doktorgrades \\ "Doctor rerum naturalium" \\ an der Georg-August-Universität Göttingen
}

vorgelegt von

Sonia Utermann

aus Rinteln

Göttingen, 2012 
Referent: Prof. Dr. Stephan Herminghaus Koreferentin: Prof. Dr. Annette Zippelius

Tag der mündlichen Prüfung: 27. Januar 2012 


\section{Contents}

$\begin{array}{ll}1 . \text { Physics in an hourglass } & 1\end{array}$

I. The frictional properties of granular media 7

\begin{tabular}{ll}
\hline Foreword to part I & 7
\end{tabular}

\begin{tabular}{ll}
\hline 2. Surface roughness & $\mathbf{1 5}$
\end{tabular}

2.1. Etching glass grains . . . . . . . . . . . . . . . . . . . 16

2.1.1. Grain sizes . . . . . . . . . . . . . . . . . . . . . . . 18

2.2. Measuring grain roughness . . . . . . . . . . . . . . . . . . . . . . 19

2.2.1. Roughness analysis . . . . . . . . . . . . . . 20

2.2.2. Radial averaging to test for aspherical grains . . . . . . . . 25

2.3. Roughness results . . . . . . . . . . . . . . . 26

2.4. Atomic force microscopy on grains . . . . . . . . . . . . . . . . 30

2.5. Using a carbonated soft drink to measure the roughness of grains 32

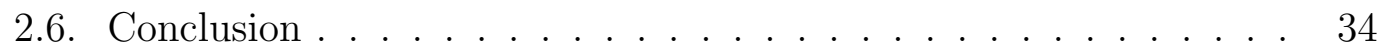

\begin{tabular}{ll}
\hline 3. Bulk friction & 37
\end{tabular}

3.1. Angle of repose . . . . . . . . . . . . . . . . . . . 37

3.1.1. Results . . . . . . . . . . . . . . . . . . . . . . . . 39

3.1.2. Pile relaxation time . . . . . . . . . . . . . . . . 41

3.2. Angle of internal friction . . . . . . . . . . . . . . . . . . . . 42

3.2.1. Asphericity, contact number and volume fraction . . . . . 44

3.3. Angle of maximum stability . . . . . . . . . . . . . . 45

3.3.1. Results . . . . . . . . . . . . . . . . . . . . 48

3.4. Conclusion . . . . . . . . . . . . . . . . . . . . . . . . 48

II. Measuring granular dynamics with light transport $\quad 55$

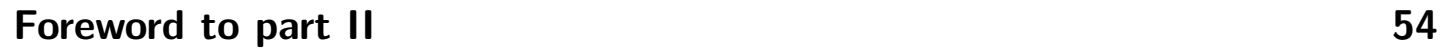

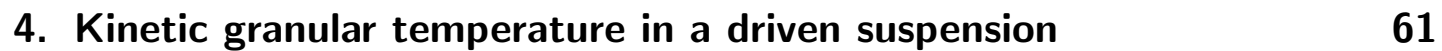

4.1. Fluidised bed . . . . . . . . . . . . . . . 61

4.2. Methods to measure $T_{\text {kin }} \ldots \ldots \ldots$. . . . . . . . . 64 
4.3. Measuring $T_{\text {kin }}$ using diffusing wave spectroscopy $\ldots . . . .65$

\begin{tabular}{|ll} 
5. Transport mean free path of light & 69
\end{tabular}

5.1. Methods of measuring $l^{*} \ldots \ldots \ldots$. . . . . . . . 70

5.1.1. Measuring $l^{*}$ in transmission . . . . . . . . . . . . 70

5.1.2. Measuring $l^{*}$ by coherent backscattering . . . . . . . . 74

5.1.3. Measuring $l^{*}$ by incoherent backscattering . . . . . . . . 76

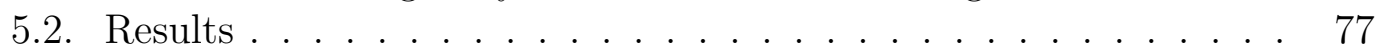

5.2.1. Transmission results . . . . . . . . . . . . 78

5.2.2. Coherent backscattering results . . . . . . . . . . 81

$5.2 .3 . \quad$ Incoherent backscattering results . . . . . . . . . . . 84

5.3. Discussion . . . . . . . . . . . . . . . . . 86

5.4. Conclusions . . . . . . . . . . . . . . . . . . 90

III. Outlook and Summary 93

6. Outlook 95

6.1. Roughening and inhomogeneity . . . . . . . . . . . . . . . 95

6.2. Wetting and roughness . . . . . . . . . . . . . . . 96

6.3. Other granular temperatures . . . . . . . . . . . . . . . . . . . . 97

6.4. An ideal granular material . . . . . . . . . . . . . . . . 100

6.4.1. Polymer spheres and the physics of packings . . . . . . . . 101

$6.4 .2 . \quad$ Interparticle forces . . . . . . . . . . . . . . 102

6.4.3. An intermediate system . . . . . . . . . . . . . 103

\begin{tabular}{ll}
\hline 7. Summary & 107
\end{tabular}

$\begin{array}{lr}\text { 8. Appendix } & 109\end{array}$

A. Side walls and flowing granular media . . . . . . . . . . . . . . . . 109

B. Principles of shear testing . . . . . . . . . . . . . . . . . 111

C. Diffusing wave spectroscopy (DWS) . . . . . . . . . . . . . . 116

D. Photodiode sensor . . . . . . . . . . . . . . . . . . . 116

E. Mie scattering . . . . . . . . . . . . . . . . . . . 117

\begin{tabular}{lr}
\hline Bibliography & 120
\end{tabular}

\begin{tabular}{ll}
\hline Acknowledgements & 128
\end{tabular}

\begin{tabular}{lr}
\hline Publications & 130
\end{tabular} 


\section{Physics in an hourglass}

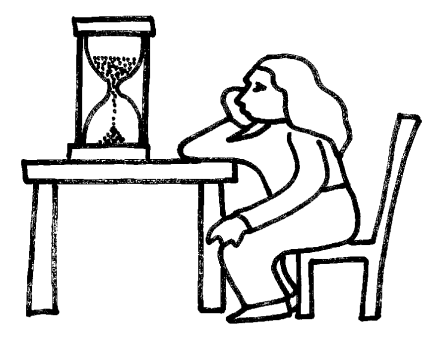

The scientist wishing to learn about granular physics could do no better than to begin her studies with the humble hourglass.

She starts by turning the hourglass, so that the sand is in the top bulb and it starts to rain through the neck into the bottom bulb. She sees three types of granular behaviour: a dense flow through the neck; followed by a granular gas in free-fall; then a sedimented pile in the bottom bulb.

Let our scientist first look at the dense flow of the grains out of the upper bulb. Depending on the ratio of the grain size to the neck diameter, the flow of grains may be steady, intermittent or even stop entirely whilst the bulb is still full [1]. The arrest of flow is caused by granular arching, and the analogy to man-made arches and domes is a justified one, as is illustrated in experiments on a two-dimensional silo (figure 1.1). The onset of granular arching for perfectly frictionless spheres occurs at a neck width of about six grain diameters, but the onset can occur for much wider necks for real, frictional grains [2].

If the neck is wide enough to prevent granular arching, the scientist observes that the flow rate is constant, regardless of the amount of sand left in the top bulb. She knows that this is not the case for a water clock. The explanation most frequently used is that this is because the vertical pressure in a granular cylindrical column reaches a saturation at a certain depth, an observation made by Hagen in 1852 [3, 4] and independently by Janssen in 1895 [5, 6], and named by convention the Janssen effect 1 This line of reasoning, however, assumes that the pressure governs the flow rate of granular materials in the same way it does in liquids. In fact, the mass flow rate through an aperture, $W$, is independent of pressure [1] and is instead governed by the empirical Beverloo law [7], which takes the form

\footnotetext{
${ }^{1}$ The Janssen effect is illustrated in the appendix in figure A2.
} 


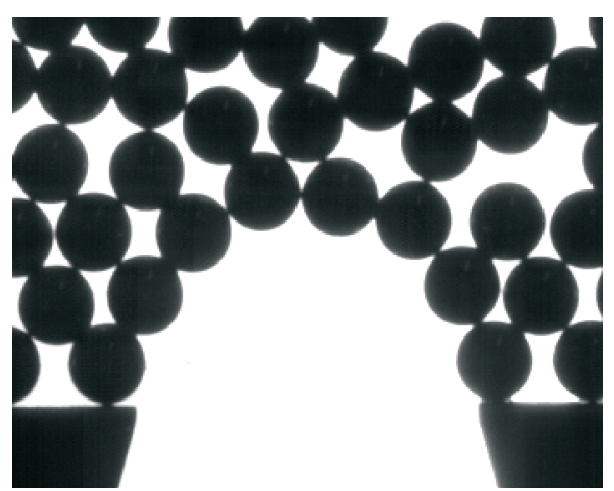

Figure 1.1.: A granular arch of $1 \mathrm{~mm}$ stainless steel spheres formed at an aperture. Taken from reference [2].

$$
W=C \rho \sqrt{g}(D-\kappa d)^{5 / 2},
$$

where $\rho$ is the material density of the grains, $g$ is the acceleration due to gravity, $D$ is the diameter of the aperture and $d$ is the (spherical) diameter of the grains. $C$ and $\kappa$ are empirical constants accounting respectively for the surface properties of the grains and the boundary layer at the orifice [1, 17. This means that $W$ is a constant whose value is determined solely by the dimensions of the aperture and the properties of the grains: friction once more playing a key role.

Now let the scientist devote her attention to the stream of falling grains. A kinetic granular temperature, $T_{k i n}$, can be defined as the kinetic energy of the random motion of the grains in a frame of reference moving at the same velocity as the stream. In the stream, the grains are subject to multiple inelastic collisions, which means that each collision reduces their kinetic energy. At first, the falling grains undergo uniform "cooling". Further down from the neck, our scientist might observe that the stream of falling grains breaks up into "droplets" similar to the Plateau-Rayleigh instability in liquids [8, 9]; this is shown in figure 1.2 . The physical origin for the cooling and the droplet formation is the loss of energy the grains suffer when they collide. The falling grains can be described as a freely cooling granular gas with a restitution co-efficient, $\epsilon$, of less than one. Any fluctuations in the density of the "gas" lead to an enhanced collision rate between the grains. Since each collision costs the grains involved $1-\epsilon$ of their kinetic energy, this leads to correlations in the grain velocities and thence to spontaneous clustering of grains, even in the absence of attractive forces. For the grains in the hourglass, surface roughness and cohesive forces such as Van der Waals and liquid bridges make this effect much more pronounced.

Finally, the scientist turns her attention to the sand in the lower bulb. It forms a growing conical heap with an angle $\alpha$ to the horizontal that changes but is always somewhere within the range $\alpha_{r} \leq \alpha \leq \alpha_{m s}$. What is the nature of the 


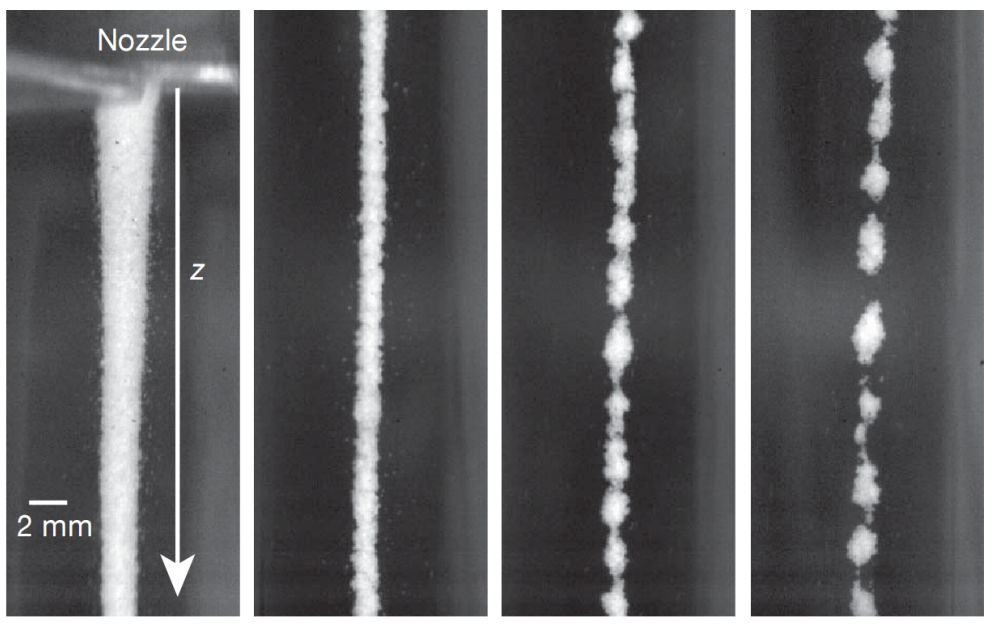

Figure 1.2.: Glass grains in free fall showing clustering. This figure, taken from reference [9], shows a series of snapshots of $3 \mathrm{~cm}$ of the granular stream taken just beneath the outlet and then at distances of $20 \mathrm{~cm}, 55 \mathrm{~cm}$ and $97 \mathrm{~cm}$ from the outlet.

two angles, $\alpha_{r}$ and $\alpha_{m s}$ that bound this range? $\alpha_{m s}$ is the angle of maximum stability and a pile with this angle is in a critical state: the slightest disturbance could trigger an avalanche. $\alpha_{r}$ is the angle of repose and this is the angle of the pile after an avalanche [10]. Coulomb was the first to suggest that $\alpha_{r}$ is a measure of the inter-particle friction. In nature, the inequality $\alpha_{r}<\alpha_{m s}$ is cleverly exploited by the antlion in digging its traps. The antlion buries itself in dry sand in a conical hole whose sides have an embankment angle very close to $\alpha_{m s}$ (figure 1.3p. When an ant wanders into the hole it triggers an avalanche that sweeps the unsuspecting insect to its doom in the hungry jaws below.

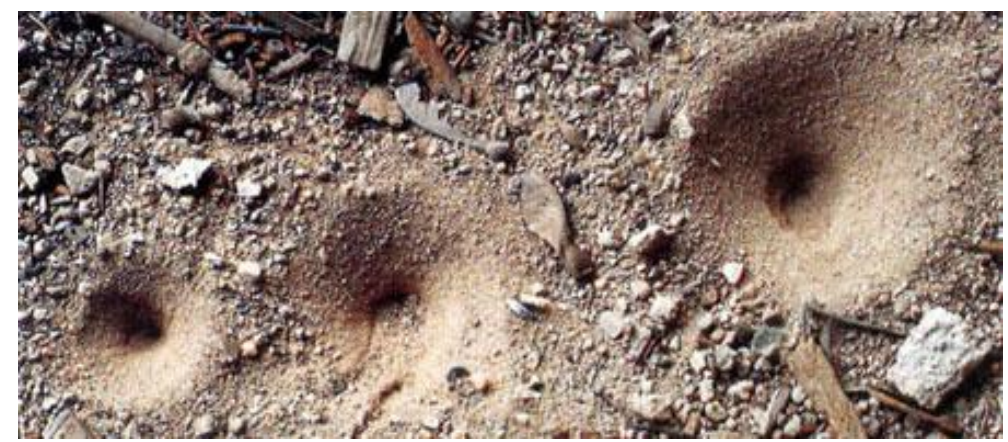

Figure 1.3.: Pits dug by the antlion, larva of the myrmeleontidae family, for catching ants. Photograph reproduced with the kind permission of Ignac Sivec, Slovenian Museum of Natural History.

Once the all of the sand has landed in the bottom bulb and is sitting in a heap at the angle of repose, our scientist might now be tempted to think that there 
is nothing else interesting to see. What could be more mundane than a heap of sand doing nothing? But static sand piles are every bit as interesting - from a physical perspective - as dynamic ones. The pile in the hourglass has a volume fraction (also called packing density) given by

$$
\phi=\frac{V_{\text {grains }}}{V_{\text {total }}},
$$

where $V_{\text {grains }}$ is the volume of the grains and $V_{\text {total }}$ is the total volume of the pile. For a random arrangement of spherical grains, $\phi$ has an upper bound of 0.64 [11, 12] and a friction-dependent lower bound of 0.55 [13, 14]. In fact, there is more to both of these "bounds" than meets the eye: the upper bound depends on how you define random [15] and the lower is strongly dependent on the friction between grains. Both are topics of vehement debate amongst granular scientists.

The density of a granular pile also depends on how it was prepared; we say it is protocol dependent. If our scientist continually taps her finger on the hourglass while it is running, she will create a packing with a greater $\phi$ than if she had just let the sand flow. She will have caused the sand pile to become more compact.

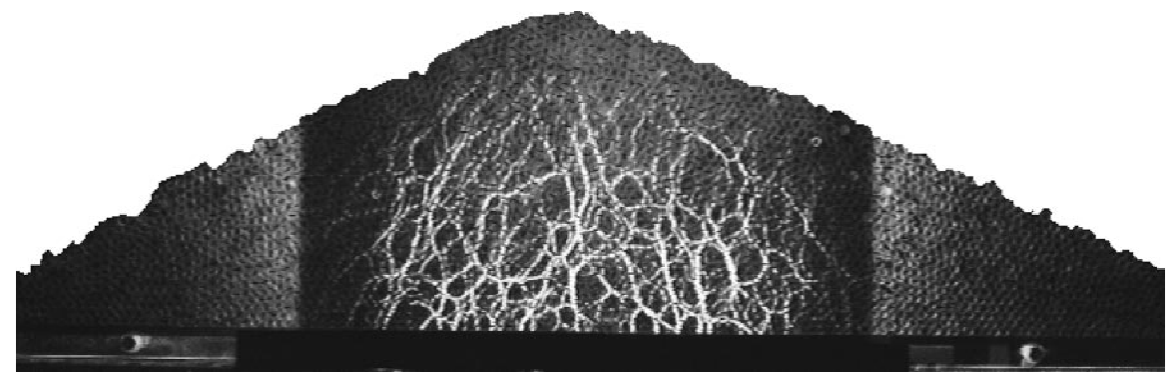

Figure 1.4.: A two-dimensional pile of discs made from a photoelastic material, whose polarisation properties change when it is subjected to stress. The centre section of the image, with a height of $30 \mathrm{~cm}$, is viewed between crossed polarisers, revealing the underlying stress structure as a network of bright branches. Note the heterogeneity of the forces: some are strong, some quite tenuous. Image taken from [16].

The volume fraction, $\phi$, is not the only static variable that is protocol dependent. The sand pile has the shape it does because it is held together by a heterogeneous network of forces that exactly balance. By tapping the glass, the scientist causes minuscule re-arrangements in the positions of grain contacts that alter the force network entirely. These force networks can take extraordinary forms: one such network is shown in figure 1.4. And the consequences of these networks are just as extraordinary: for instance, we would expect that the pressure felt by the base of the hourglass should be greatest at the centre of the pile. After all, that is where the mass is the greatest. But this is not always the case, as shown in figure 1.5. In the figure, two different protocols have been used 
to prepare a granular pile. Outwardly, both piles look the same, but there is a significant dip in the pressure at the centre of one of the piles, and this stems from the fact that the force networks form arches - similar to the arch in figure 1.1 that distribute the effective weight of the pile differently in the two cases. One of the keys to understanding the mechanics of a sand pile is to visualise and understand the force network that is keeping it standing.
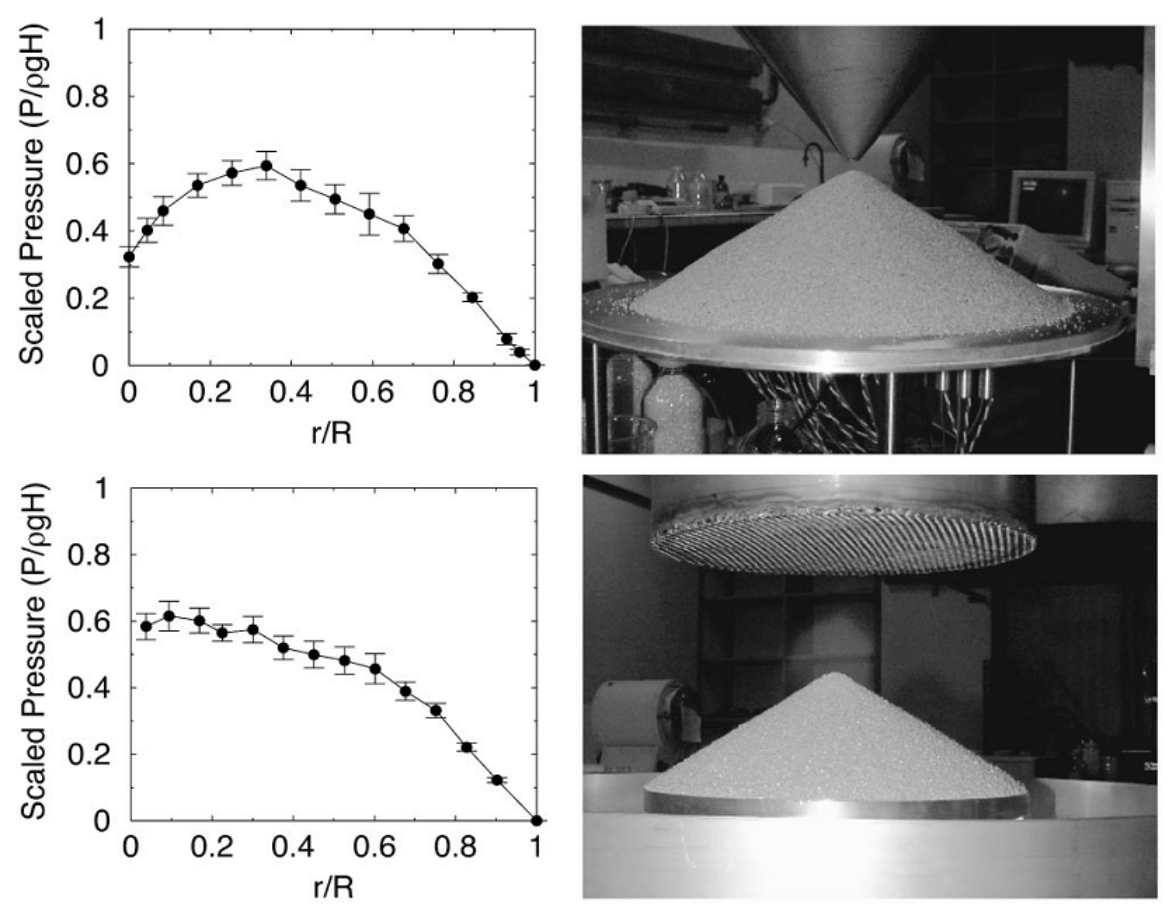

Figure 1.5.: Right hand side: two sand piles formed by different preparation protocols. The top pile was formed by raining sand through a funnel onto a base plate, the same mechanism as in the hourglass. The bottom pile was formed by raining sand through a sieve onto a pedestal. Left hand side: Dimensionless normal pressure profiles, $P / \rho g H$, as a function of dimensionless radial position, $r / R$, for both piles. $r / R=0$ is the centre of the pile, 1 its edge. The funnel-fed pile has a dip in the pressure at the centre of the pile; the sieve fed pile does not. Figure taken from [16].

In turning the hourglass, the scientist has injected energy - in the form of potential energy - into the system. In doing so, she allows the initially "solid" sand to flow in a liquid fashion; from a thermodynamics point of view, she has caused the sand to undergo a change of state - to "melt" - and has raised the "temperature". Once the sand is flowing through the neck, the grains undergo multiple inelastic collisions and the sand "cools down". When it hits the bottom of the hourglass, the sand is once again stationary, as if it had frozen. "Melting", "cooling" and "freezing", yet a thermometer buried in the sand would not register any difference in temperature. The temperatures and changes of state observed 
in a granular medium are of a different type to the ones familiar to us from equilibrium thermodynamics, but we cannot resist making the analogy. Is our scientist allowed to apply her knowledge of equilibrium thermodynamics to the sand in her hourglass? A framework of statistical mechanics of static granular media has been suggested in which she may ${ }^{2}$ However, perhaps more intuitive than the static thermodynamics of Edwards and Oakeshott is a definition of a granular temperature developed as an extension of the kinetic theory of gases. In this formalism, the granular temperature is defined simply as the kinetic energy of the random motion of the grains [18, 19]. $]^{3}$

In her study of the hourglass, the scientist has made acquaintance with all of the granular physics that will be encountered in this thesis.

In the dense flow of sand through the neck, the granular gas of freely-falling grains and in the sedimented pile in the bottom bulb, she saw that friction plays a key role in all phenomena. Granular friction -and how it can be systematically tuned- is the subject of part I. In the dense flow and the granular gas, she saw that it makes sense to speak of a kinetic granular temperature. Part II deals with granular temperature in dense flows and how it can be measured optically. Finally, in the stationary pile, the forces between grains interested her. Section 6.4 looks at how these forces can be measured.

${ }^{2}$ In 1989, Edwards and Oakeshott [17] suggested a framework of statistical mechanics of static granular media in which they define a compactivity variable, $\chi=\frac{\partial V}{\partial S}$ as an analogue to the temperature $T=\frac{\partial E}{\partial S}$ in equilibrium thermodynamics, where $V$ is the volume, $S$ the entropy and $E$ the energy.

${ }^{3}$ For an ideal gas, $E_{\text {kin }}=\frac{2}{3} k_{B} T=\left\langle\frac{1}{2} m v^{2}\right\rangle$, where $E_{\text {kin }}$ is the kinetic energy, $k_{B}$ Boltzmann's constant, $T$ temperature, $m$ particle mass and $v$ the particle velocity. In the formalism of the kinetic theory of granular media, $k_{B}$ has no meaning and rotational degrees of freedom need to be accounted for. The unit of $T_{k i n}$ is Joule [18, 19]. 


\section{Part I.}

\section{The frictional properties of granular media}





\section{Foreword to part 1}

In the introduction, we encountered a number of phenomena in granular statics and dynamics in which interparticle friction is one of the key physical mechanisms. In fact, second only to excluded volume, friction is the most important mechanism in determining the physical behaviour of granular materials [10]. In the next two chapters, we will look at the outcome of an attempt to tune friction in granular media by changing the surface roughness of grains.

In chapter 2, a chemical etching procedure is explained by which the surfaces of glass grains can be modified. The grain surface profiles are measured using interferometry and atomic force microscopy and their roughness is characterised statistically. A method for measuring roughness in bulk granular samples is demonstrated. Then, in chapter 3, we go on to test the frictional properties of the etched grains. Their coefficient of friction is measured (1) by looking at their angle of repose in a sedimentation experiment that is essentially an hourglass; and (2) in a shear cell. The effect of inter-particle friction on volume fraction, $\phi$, is also investigated.

But first, let us look at what friction is, particularly in the context of granular media.

\section{The physics of friction}

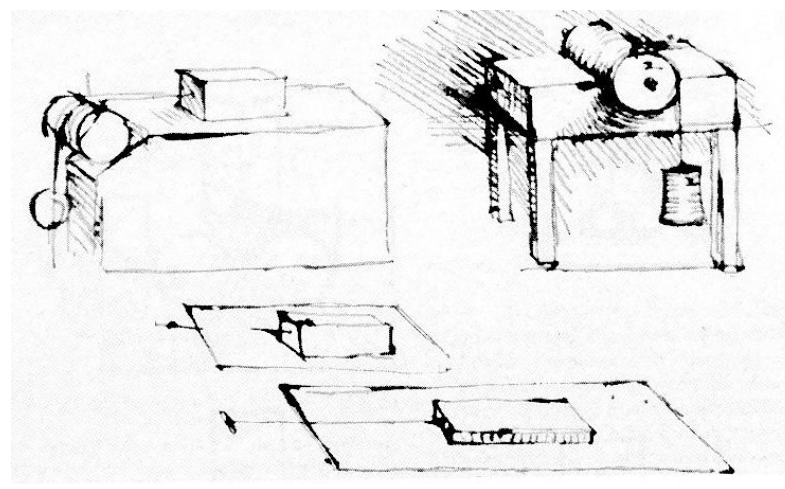

Figure 1.6.: Sketches from da Vinci's notebook (ca. 1480), demonstrating some of his friction experiments 20 . 
Until recently, the physics of macroscopic friction was a purely empirical science [21]. One of the first people to study friction systematically was da Vinci (14521519), who noted two fundamental laws for friction in his journal (figure 1.6). He observed that (1) the frictional force is proportional to the mass of the body and that (2) the friction between a sliding body and a surface is independent of the contact area [10]. However, he never published his observations. The same two laws were discovered independently in 1699 by Amontons [22] and expanded in 1776 by Coulomb [23], who noted the first law in the form

$$
F_{f}=\mu F_{N},
$$

where $F_{f}$ is the frictional force between two bodies and $F_{N}$ is the normal force between them. $\mu$ is the coefficient of friction. Coulomb was the first to apply the principles of solid friction to granular media. His law of granular friction, as paraphrased by Duran [10], says that

two contiguous sheets of granular material cannot slide relative to each other unless their inclination $\theta$ is at least equal to $\tan ^{-1}(\mu)$.

In analogy with solid friction, the granular friction coefficient, $\mu$, is to be seen as a material constant.

In 1750, Euler observed that friction is hysteretic, and as such, one needs to discriminate between static and dynamic friction. He introduced a third law of friction: that the coefficient of dynamic friction is less than or equal to the coefficient of static friction [24].

An explanation of the laws of friction based on plasticity and adhesion was postulated by Bowden and Tabor in 1942 [21]. By electrical resistance measurements, they determined that the true area of contact is only a fraction of the apparent contact area. The true contact area is formed by the $\mu \mathrm{m}$-scale asperities. As the normal force increases, more asperities come into contact and the average area of each contact grows. Furthermore, contacts can be plastically deformed [25]. They showed that, for two metal surfaces of differing hardness, the frictional force is dependent on the true contact area according to

$$
F_{f}=S_{s o f t} A=\frac{S_{\text {soft }} F_{N}}{p},
$$

where $S_{\text {soft }}$ is the shear strength of the softer metal, $A$ is the true contact area (which can be $10^{3}$ smaller than the apparent contact area), and $p$ is the pressure at which the asperities deform. The static coefficient of friction is therefore a material constant of the softer metal, $\mu_{s}=S_{\text {soft }} / p$ [21]. The authors made no allowance for roughness, which is why they assume that $A=F_{N} / p$ for all surfaces. Intuitively we might expect this to be a proportionality, rather than an equality, since the rate at which $A$ increases with increasing load should depend on the type of asperity on the surfaces. 
Our everyday experience tells us that rough surfaces have higher friction than smooth surfaces. The question of roughness and friction was first addressed systematically by Bikerman in 1944 and in 1949 in two articles about sliding and rolling contacts. He argued that plasticity and adhesion play only a small role in frictional behaviour under "ordinary" conditions, since (a) the contact points between two solids are usually separated by a film of gas or liquid of negligible shear strength, $S$; and (b) the observed reversibility of $F_{f}\left(F_{N}\right)$ does not allow for adhesion. In fact, the absence of a satisfactory explanation for what he calls "everyday friction" (the friction of rough surfaces) led him to the statement that

if one employs the term friction in the loose sense of the everyday usage, there exists no law of friction and no general mechanism for it.

However, his experimental findings were solidly conclusive: he found that for both sliding and rolling metal surfaces, increasing the roughness led to an increase in the friction coefficient [26, 27].

Since this date, there have of course been many attempts to find a general explanation for why and how roughness influences friction: it is, after all, an important mechanical engineering question. But the proliferation of articles on roughness and dry friction in the tribology literature suggests that Bikerman's exasperated statement still holds.

\section{Friction in granular statics}

Let us consider the simplest frictional granular system: a single sphere. We place this sphere in a wedge (figure 1.7). What are the forces acting on the sphere? In fact, there is not a unique theoretical answer to this question. The forces experienced by the sphere in an individual realisation of this experiment depend on which of the walls the grain touched first, as well as the relative amounts of sliding and rolling the grain underwent in reaching the stable position at the bottom of the wedge. Therefore the forces at a static contact are history dependent. They are inherently indeterminate.

If we expand this problem to one of a regular, "crystalline" packing of spheres, the problem becomes virtually intractable. Even in the case of frictionless spheres, microscopic asperities will lead to a certain randomness in the spatial arrangement of the contact points. If we now add friction to the arrangement, we can see that even identical packings will be stabilised by different force networks, depending on the exact, microscopic way the packing was formed [10]. An example of this phenomenon can be found in the work of Tighe et al., shown in figure 1.8.

Random packings get even more complicated. Multiple realisations of a random packing of frictionless convex grains will all have the same volume fraction; in the case of monodispers $\varrho^{4}$ hard spheres, the volume fraction will always be about 0.64

\footnotetext{
${ }^{4}$ spheres of the same size
} 


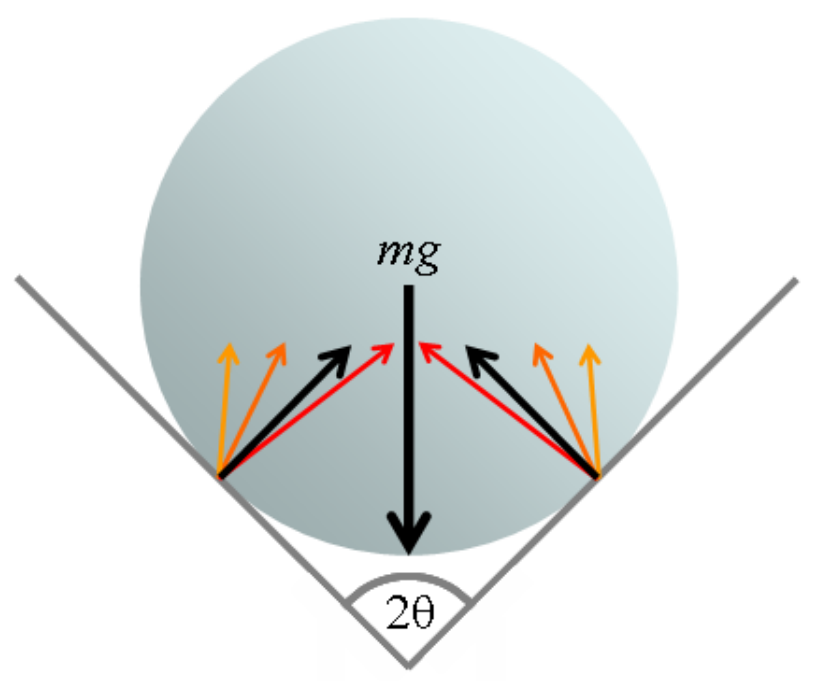

Figure 1.7.: $\quad$ A sphere of mass $m$ in a wedge of opening angle 2 $\theta$. In the absence of friction, the sphere is supported by the two normal forces $F_{n}=\frac{1}{2} m g \sin \theta$ (black arrows). Introducing frictional (tangential) forces allows any number of ways of balancing the forces (coloured arrows). Figure adapted from reference [28.

[11. This is referred to as the random close packing limit, RCP. Volume fractions above this limit will always contain some spontaneous crystalline ordering [11, 12, 15. Frictional grains produce random packings across a range of volume fractions; reference is often made to a random loose packing limit (RLP), and random packings can be produced at any volume fraction, $\phi$, between $\phi_{R L P}$ and $\phi_{R C P}$. Debate is still raging as to what limit(s) $\phi_{R L P}$ should take even for the case of perfect spheres, since the random loose packing volume fraction decreases with increasing inter-particle friction coefficient [13, 14], and some assert that even $\phi_{R C P}$ cannot be defined [29].

\section{Friction in granular dynamics}

Perfectly smooth particles will slide past one another rather than roll. In a dense packing of rough grains, virtually all rotation modes may be frustrated, which could also lead to purely sliding contacts. Between these two extrema, grains will roll and slide at the contact points, and the proportion of rolling to sliding will depend on the degrees of roughness and frustration [10].

Grains in an agitated system also collide, and the energy dissipated in a collision is usually described by the coefficient of restitution, $\epsilon$. It is more accurate to speak of two coefficients of restitution: one for the normal component and one for the tangential component of a non-frontal collision. Since the coefficient of tangential 


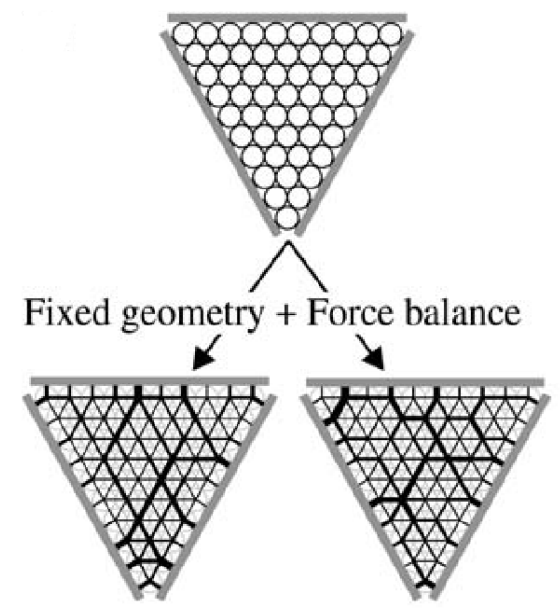

Figure 1.8.: Identical ordered packings of discs can satisfy force balance in different ways. The black lines show the force network; the thicker the line, the greater the force. Figure taken from reference [28].

restitution depends on the coefficient of friction $5^{5}$ it is easy to see how changing the roughness of grains can change their collision dynamics [32, 33, 34, 35].

Experiment has proven the vital role friction plays in granular dynamics: for instance in the case of a granular material undergoing shear. The effect of friction in granular materials under shear in a linear, split-bottomed shear cell filled with alternating layers of high-friction and low-friction grains has been investigated by Börszönyi et al. [36]. Here, it was shown that the position of the shear band depends on the layering of the two types of grains. In a different shear set-up, Anthony and Marone found that increasing particle roughness and friction led to an increase in the loading a granular packing could withstand [37]. They saw that stick-slip characteristics were different for rough and smooth grains, and suggested that this implies a different network of force chains.

It has been shown experimentally that friction can also play an important role in granular segregation, for example in the sharp transition from the reverse brazil-nut effect to the brazil-nut effect in a shaken granular sample [38].6 The dynamical properties of granular avalanches have been shown to depend on inter-particle friction, too: Börszönyi, Halsey and Ecke identified two frictiondependent scenarios for granular avalanches on an incline [40, 41, 42].

In the following two chapters of this thesis, it is shown that it is possible to tune the topographic properties of soda-lime glass grains, making inter-particle

\footnotetext{
${ }^{5}$ Even this is a simplification. It has been shown that the coefficients of restitution also depend on the velocities of the grains [30, 31.

${ }^{6}$ The "brazil-nut effect" is the phenomenon that leads to the larger particles moving to the top of a shaken mixture of small and large particles. In a rotating drum experiment, the friction dependence was not observed [39].
} 
friction a control parameter for some types of grains. This allows the systematic quantitative study of the principles underlying granular phenomena such as those mentioned above. 


\section{Surface roughness}

The important role that friction plays in granular physics demands a systematic experimental study in which the inter-particle friction is a control parameter. The current chapter is an attempt to address this breach. In common with most other experimental work on granular physics, we take soda-lime glass spheres as our granular medium. Our goal is to tune the inter-particle friction by changing the roughness of the grain surface.

Apart from the ability to use friction as a control parameter in fundamental physical experiments, tuning the roughness of grains allows us to tune the wetting of and adhesion between grains in the presence of moisture [43. This is discussed in detail in section 6.2. There may also be a number of industrial applications of the roughening technique presented in this chapter. One such is in granular fluidised or packed-bed filters that are used for the biological filtering of water. Here, changing the roughness of the grains alters the surface available for ammonia-fixing bacterial films without the need to change the grain size, which would affect the filtering rate [44]. Fluidised or packed-bed reactors such as the catalytic cracking units used in the oil industry might well be further optimised for particular specialisations if the roughness of the grains can be tuned. Maas et al. [45] suggest an application of tuned roughness in their study of the powdered drug-carrier mannitol: $]^{1}$ in their case they wish to tune the particle-particle and drug-particle contact areas.

Our approach is summarised as follows: we present two chemical etching procedures with which we treat soda-lime glass spheres. Figure 2.1 shows the surfaces of grains when they are untreated and after etching with the two protocols. We produce 21 batches of variously treated spheres. We then characterise their surfaces using vertical-scanning white-light interferometry (WLI) and atomic force microscopy (AFM), obtaining the roughness length scales and the exponent that uniquely describe the topography of the surface.

\footnotetext{
${ }^{1}$ The authors suggest using spray-dried mannitol grains to replace the lactose currently used as the carrier in powder inhalers. They say that mannitol is less prone to trigger allergic reactions [45].
} 


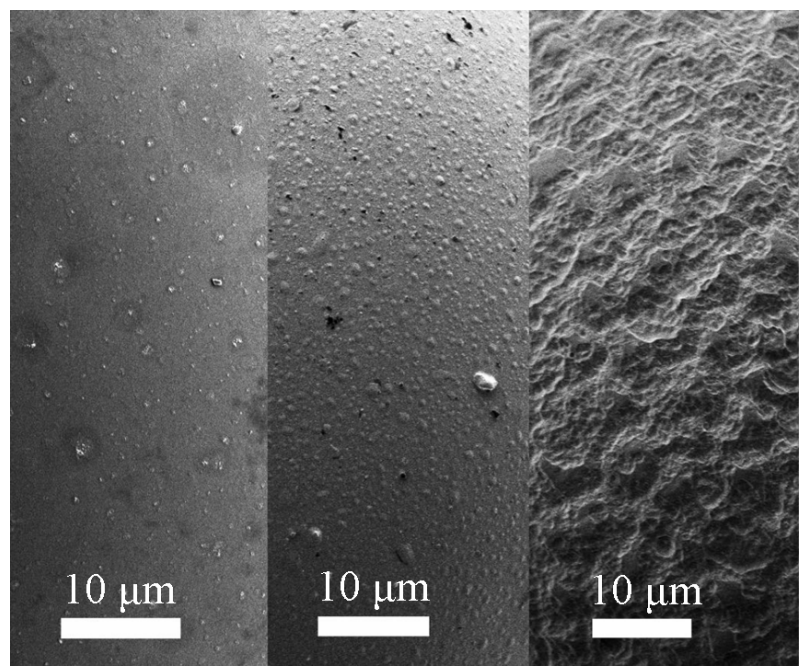

Figure 2.1.: Scanning electron microscopy (SEM) images of the surfaces of sodalime glass spheres $146 \mu \mathrm{m} \pm 19 \mu \mathrm{m}$. Left: smoothed by $\mathrm{NaOH}$. Centre: untreated. Right: etched for four minutes in $\mathrm{NH}_{4} \mathrm{FHF}$. Microscopy by K. Sand, University of Copenhagen.

\subsection{Etching glass grains}

\section{Roughening protocol}

Usually, hydrofluoric acid (HF) is used to etch glass [46, 47]. However, HF alone is unpleasant to work with and is harmful to the environment [48]. Furthermore, our experiments with dilute HF showed highly inhomogeneous etching unconducive to our purpose, as demonstrated in figure 2.2. We developed an etching protocol based on ammonium bifluoride, $\mathrm{NH}_{4} \mathrm{FHF}$, that showed better etching properties (figure 2.1(right)) as well as better handling: equation 2.1 (top) shows that hydrofluoric acid is produced in proportion to the amount of water in the solution. This means that the amount of HF can be kept to a minimum by using only a small amount of water and adding glycerine as a liquid carrier.

$$
\begin{aligned}
\mathrm{NH}_{4} \mathrm{FHF}+\mathrm{H}_{2} \mathrm{O} & \rightleftharpoons \mathrm{NH}_{4}^{+}+\mathrm{FHF}^{-} \\
\mathrm{FHF}^{-} & \rightleftharpoons \mathrm{HF}+\mathrm{F}^{-} \\
4 \mathrm{HF}+\mathrm{SiO}_{2} & \rightarrow \mathrm{SiF}_{4}+2 \mathrm{H}_{2} \mathrm{O}
\end{aligned}
$$

Our roughening recipe optimises the flow properties of the etch solution whilst etching as homogeneously as possible and releasing little hydrofluoric acid [49]. The directions are as follows.

1. 14 parts by mass of liquid glycerine, $\mathrm{C}_{3} \mathrm{H}_{8} \mathrm{O}_{3}$, and six by mass of distilled water are heated in a water bath to $90^{\circ} \mathrm{C}$. 


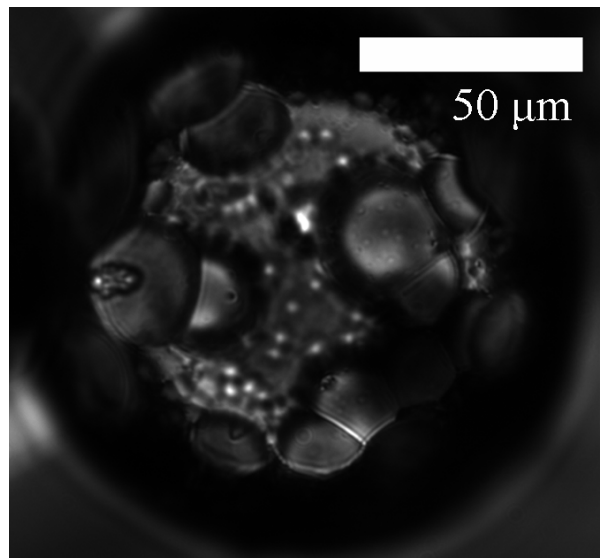

Figure 2.2.: Optical micrograph of a soda-lime glass sphere etched in HF. Instead of homogeneous surface roughening, large craters are produced.

2. Six parts by mass of solid ammonium hydrogen-difluoride, $\mathrm{NH}_{4} \mathrm{FHF}$, and one part by mass of solid granulate iron(III)chloride-hexahydrate, $\mathrm{FeCl}_{3}$. $6 \mathrm{H}_{2} \mathrm{O}$ (a catalyst), are added.

3. An over-saturated solution is produced by mixing vigorously at $90^{\circ} \mathrm{C}$.

4. The solution is cooled under agitation until it is cool enough to be placed in the centrifuge.

5. The over-saturated solution is centrifuged until the clear solution is separated from the undissolved solute (about 15 minutes).

6. The solution is added to the glass grains and shaken vigorously. After the desired etching time has passed, the solution is poured out and the grains thoroughly washed with distilled water.

N.B. This is a hydrofluoric acid etching procedure, so care must be observed during all steps in order to avoid chemical burns. Since the procedure is one to etch glass, plastic lab ware should be used.

\section{Smoothing protocol}

A separate etching procedure to make the grains smoother was also employed. This method was published by Shellenberger and Logan [50]. To smoothen the grains, they are immersed in $12.5 \mathrm{M}$ sodium hydroxide, $\mathrm{NaOH}$, for 30 minutes, under occasional agitation. Sodium hydroxide etches only weakly at room temperature, so only the small asperities on the surface of the grain are removed at this concentration and duration. This etching protocol unfortunately did not 
bring about a significant change in the roughnes: ${ }^{2}$ of grains etched in $\mathrm{NaOH}$ compared to unetched grains.

\section{Grain types}

We use soda-lime glass grains from two different manufacturers and in two different sieve fractions. These are presented in the table below.

\begin{tabular}{|c|c|c|c|}
\hline Name & Diameter $(\mu \mathrm{m})$ & STD $(\mu \mathrm{m})$ & Symbol \\
\hline Small Mo-Sci & 146 & 19 & $\boldsymbol{\Delta}$ \\
Cataphote & 221 & 40 & $\square$ \\
Large Mo-Sci & 240 & 20 & $\bullet$ \\
\hline
\end{tabular}

Table 2.1.: The grains used in this study. Mean particle diameter and standard deviation (STD) were measured using a Camsizer, as outlined in section 2.1.1 below.

We perform seven treatments on each type of glass grain: smoothed for 30 minutes in $\mathrm{NaOH}$ ("smoothed"), as supplied by the manufacturer ("unetched") and roughened for 30 seconds and one, two, three and four minutes in $\mathrm{NH}_{4} \mathrm{FHF}$ ("roughened"). The effects of the seven treatments are illustrated in figure 2.3.

\subsubsection{Grain sizes}

The effect of etching on particle size is measured using a Retsch Technology Camsizer. This performs particle size and shape analysis based on digital imaging and it works along the following principle: a granular sample falls in front of an illuminated backdrop through the focal plane of a camera. The resulting bright-field images are processed to obtain a number of size and shape data for each grain; for us, the diameter is the relevant measure. The area of the grain, $A$, is measured and the effective diameter, $d$, of the (not necessarily spherical) grain is given by

$$
d=\frac{2 \sqrt{A}}{\pi}
$$

In 50 bins from $0 \mathrm{~mm}$ to $3 \mathrm{~mm}$, the volume-weighted particle size distribution of more than 200,000 grains of each batch is measured; a Gauss fit gives the mean and standard deviation of the particle size. This is shown in figure 2.5, for small Mo-Sci grains etched for two minutes in $\mathrm{NH}_{4} \mathrm{FHF}$.

After four minutes' etching with the roughening protocol, there is no resolvable reduction in grain diameter for the large Mo-Sci and Cataphote grains. The small Mo-Sci grains undergo a small reduction of $3.5 \mu \mathrm{m} \pm 1.5 \mu \mathrm{m}$ (a reduction of $2.4 \%$ ) after four minutes of etching. The smoothing protocol does not bring about a

\footnotetext{
${ }^{2}$ Roughness is defined in section 2.2 .1
} 


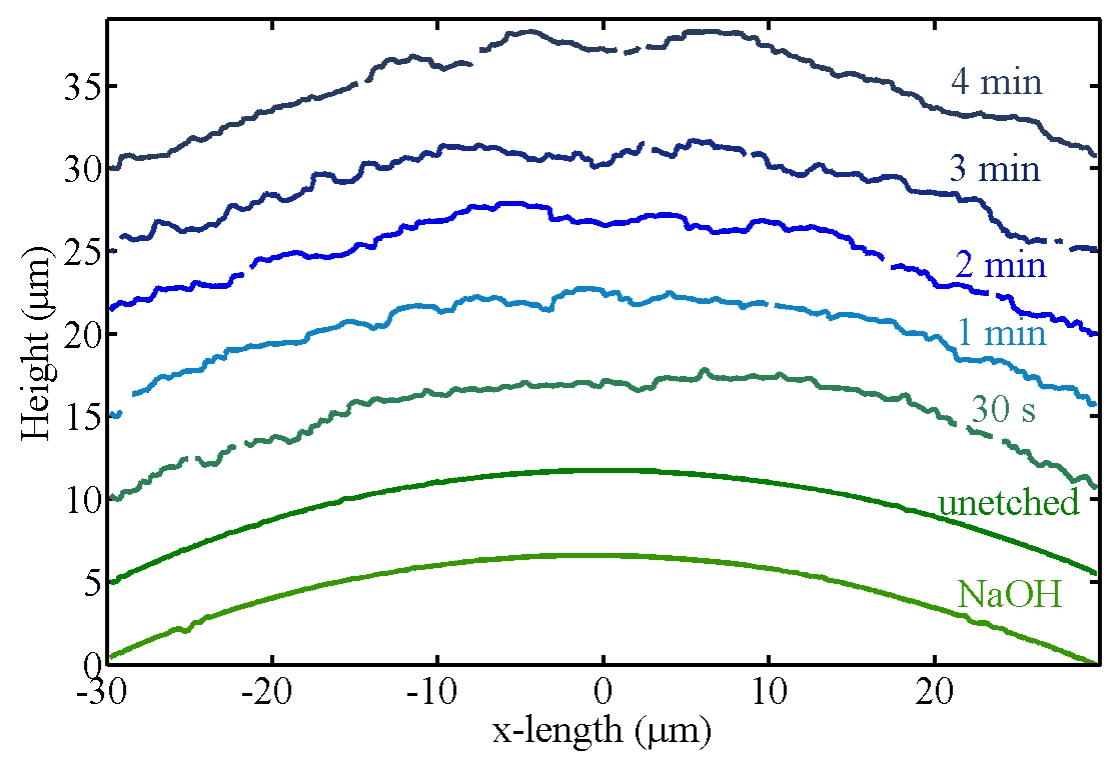

Figure 2.3.: Cross-sections of large Mo-Sci grains etched as described in the text. Profiles were measured using white-light interferometry (see section 2.2). The plots are vertically offset by $5 \mu \mathrm{m}$ for visual clarity. Gaps in the profiles are due to bad pixels in the height measurement (section 2.2 ).

resolvable reduction in diameter. The width of the size distributions also remains constant within experimental error. These results are shown in figure 2.5. We can conclude that the effect of etching on particle size is negligible.

\subsection{Measuring grain roughness}

The grain surface topography is measured using white-light vertical scanning interferometry (WLI) ${ }^{3}$ WLI provides nanometre height resolution over a large field of view. The principle of operation is illustrated in figure 2.6. After passing through an external beam splitter, the white light passes through a temperaturestabilised Mirau objective (magnification: $160 \mathrm{x}$ ). The objective is equipped with a second, internal, beam splitter. One beam is reflected by a stationary reference mirror, the other by the sample surface. The two light beams differ in optical path length as a function of the distance to the sample surface. The resulting interference pattern is captured by a high-resolution charge-coupled device (CCD). The focal plane of the interference pattern is scanned through the

${ }^{3}$ ZeMapper, Zemetrics Inc., Tucson, Arizona [51] 


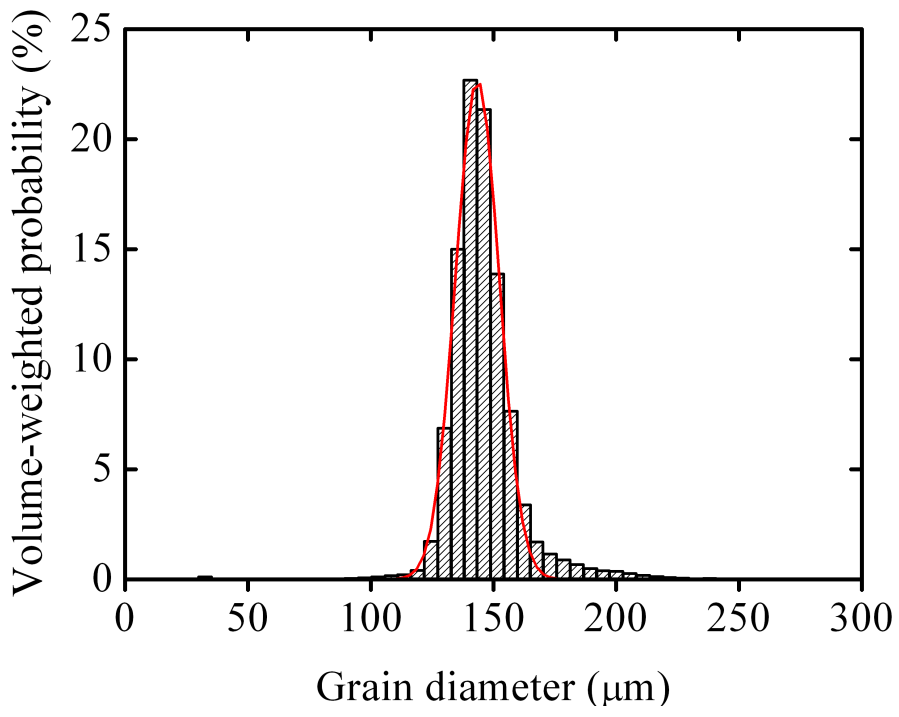

Figure 2.4.: Volume-weighted size distribution of a sample of 227,894 small Mo-Sci grains etched for two minutes. The red line is a Gauss fit to the distribution giving a mean particle diameter of $143.4 \mu \mathrm{m}$ and $\sigma=17.7 \mu \mathrm{m}$.

sample. From the collected interference pattern data, a height map is calculated. The vertical resolution of the data set is $<1 \mathrm{~nm}$, the lateral resolution is $80 \mathrm{~nm}$; the maximum field of view applied in this study is $80 \mu \mathrm{m} \times 80 \mu \mathrm{m}$ (figure 2.7).

For the WLI measurements, the sample grains are bonded to glass microscope slides with ultra-violet-curable adhesive (Norland Optical Adhesive 61) and sputter-coated with $40 \mathrm{~nm}$ gold. For each batch of grains, ten to fifteen grains are analysed $\left.\right|^{4}$

\subsubsection{Roughness analysis}

Characterising roughness is a complex endeavour in its own right, and there is a variety of tools available to do so 5 We use the height difference correlation function. This method finds a typical surface feature size characterised by a vertical, $\xi_{\text {vert }}$, and a lateral length scale, $\xi_{\text {lat }}$ as well as the Hurst exponent, $H$, which describes the roughness at length scales smaller than $\xi_{\text {vert }}$ and $\xi_{\text {lat }}$ [52].

When measuring the roughness of spheres, one is presented with additional,

\footnotetext{
${ }^{4}$ Fifteen grains are measured, but up to five are discarded because of asphericity. This is explained in section 2.2 .2

${ }^{5}$ For example, root mean squared roughness, (multi-)affine box-counting, power spectral analysis or wavelet analysis. The latter three are mostly used for fractal analysis. An overview of these methods can be obtained from references [52] and [53. The RMS roughness is the most frequently used method in geology and surface science, but it is not a unique measure.
} 


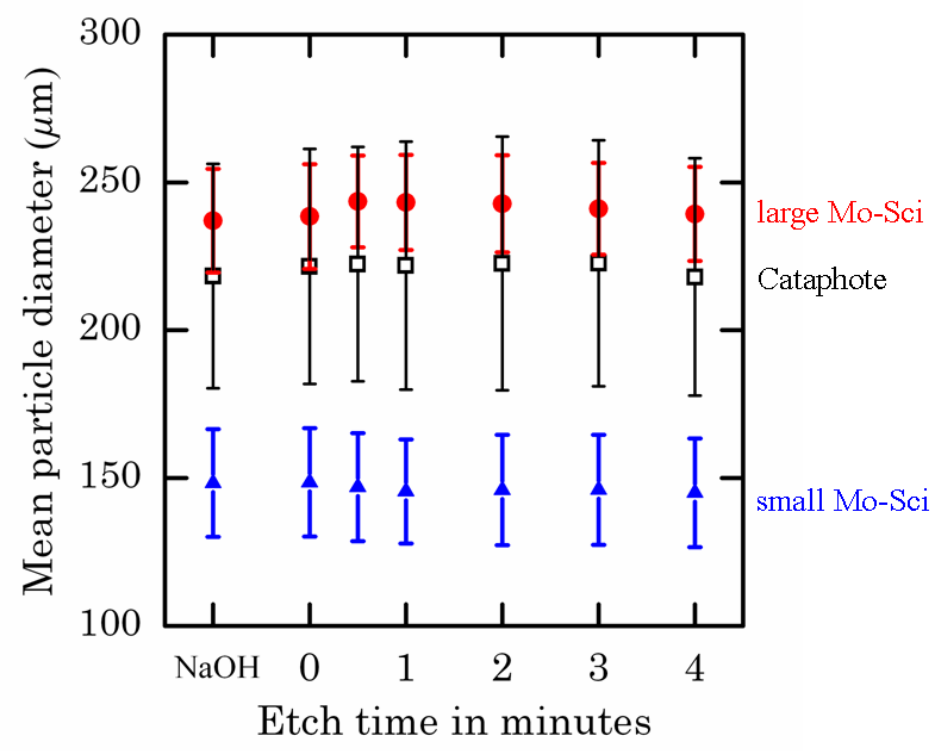

Figure 2.5.: The mean grain diameter as a function of etch time, measured for at least 200,000 grains. The grains smoothed with $\mathrm{NaOH}$ are plotted on the negative time axis. The symbols correspond to table 2.1 the error bars show the standard deviation.

unique problems. To obtain reliable statistics, a large field of view is necessary. The curvature of a spherical surface means that the measurement method must have a large enough dynamic range to be able to measure nano-scale features over a height range of several $\mu \mathrm{m}$ (figure 2.7). WLI proved to be better than atomic force microscopy (AFM) for this purpose; this is demonstrated in section 2.4 .

Once a height map, $z(x, y)$, has been obtained, this may not be treated as a plane, since it is a projection of a sphere surface onto a plane, which can never be simultaneously conformal and distance conserving [54]. It is also necessary to make an angle-of-sight correction to account for having measured height in the $z$-coordinate instead of in the radial direction. Additionally, a batch of soda-lime glass grains will not consist only of perfectly spherical grains. Our roughness analysis accounts for all of these obstacles and works as follows:

For a one-dimensional interface, $\xi_{\text {vert }}, \xi_{\text {lat }}$ and $H$ are obtained from the (secondorder) height difference correlation function, $C(L)$, given by [52]

$$
C(L)=\left\langle(h(x+L)-h(x))^{2}\right\rangle^{1 / 2},
$$

where $h$ is the height of the interface at positions $x$ or $x+L .\langle\cdots\rangle$ indicates the average for one $L$. For the surface of a rough sphere described in spherical polar coordinates, the height difference correlation becomes 


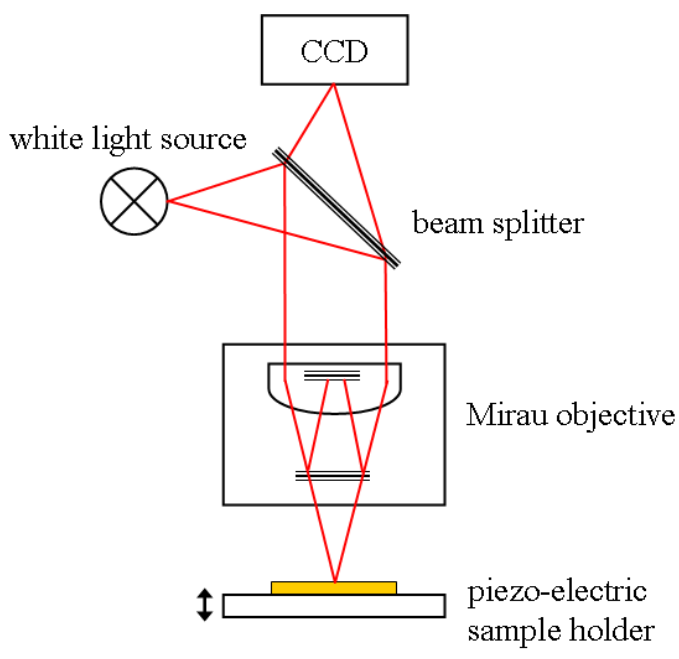

Figure 2.6.: Principle of operation of a white-light vertical scanning interferometric microscope. Image (modified) courtesy of C. Fischer, University of Göttingen.

$$
\rho(L)=\left\langle\left(r^{\prime}\left(\theta^{\prime}, \phi^{\prime}\right)-r(\theta, \phi)\right)^{2}\right\rangle^{1 / 2}
$$

where the points $r(\theta, \phi)$ and $r^{\prime}\left(\theta^{\prime}, \phi^{\prime}\right)$ are separated by the correlation interval $L$. On a sphere, $L$ is the great-circle distance between the two points calculated using the spherical cosine law:

$$
L=R \arccos \left(\cos \theta \cos \theta^{\prime}+\sin \theta \sin \theta^{\prime} \cos \left(\phi^{\prime}-\phi\right)\right)
$$

where $R$ is the sphere radius (figure 2.8).

When analysing the roughness of the glass spheres, we make an angle-of-sight correction to account for our having measured height in the $z$-coordinate instead of in the radial direction. This is shown in figure 2.9. We correct the vertical height, $\Delta z(x, y)$, to a radial feature size, $\Delta r(\theta, \phi)$, by

$$
\Delta r=\frac{\Delta z}{\cos \theta}
$$




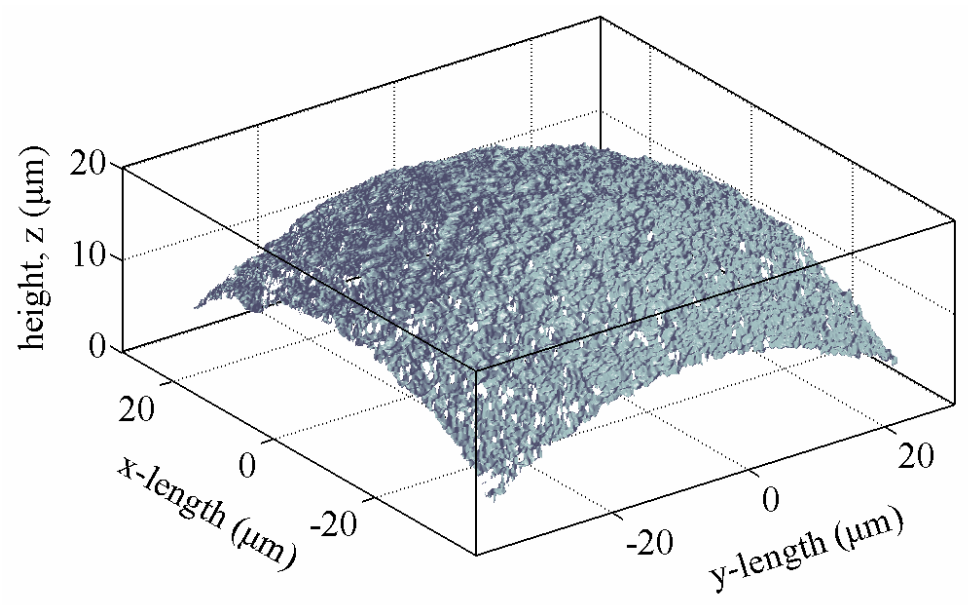

Figure 2.7.: Interferograph of the cap of a large Mo-Sci grain etched for four minutes. As well as the curvature of the grain, of the order of $\sim 100 \mu \mathrm{m}$, the fine detail of the roughness, of the order of $1 \mathrm{~nm}$, is resolved.

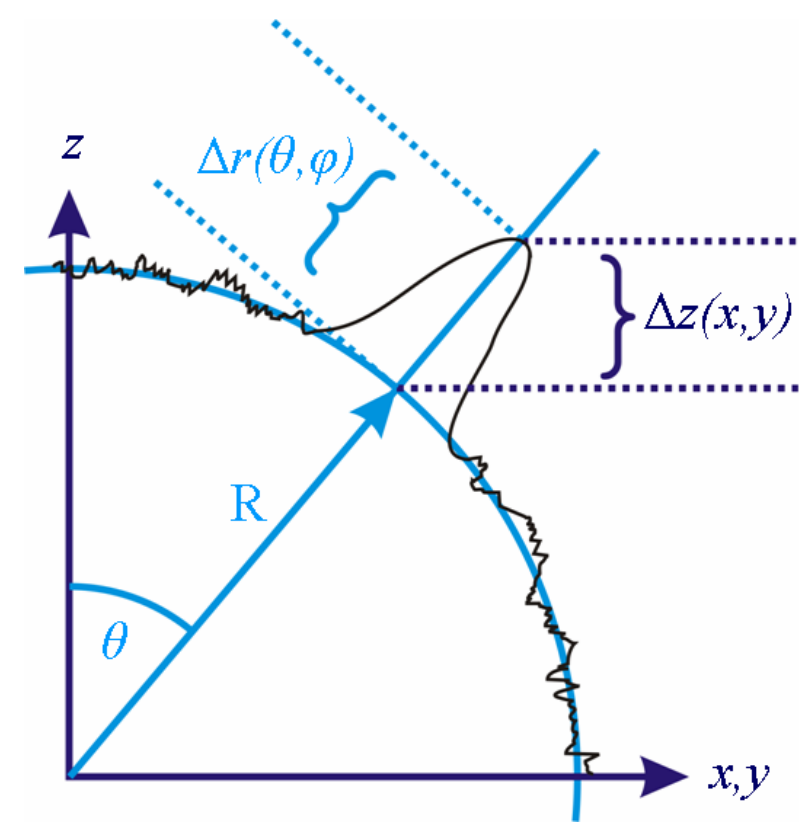

Figure 2.9.: The angle of sight correction. $R$ is the sphere radius and $\Delta z(x, y)$ is the height of the grain profile measured vertically from above. The correct radial height of the feature is given by $\Delta r=\frac{\Delta z}{\cos \theta}$.

The relevance of this correction can be estimated for the worst-case scenario: a feature at the outermost corner of the measured region of interest on a small Mo-Sci grain. In this case, $\theta=37^{\circ}$; thus omitting this correction results in an error of $26 \%$. 

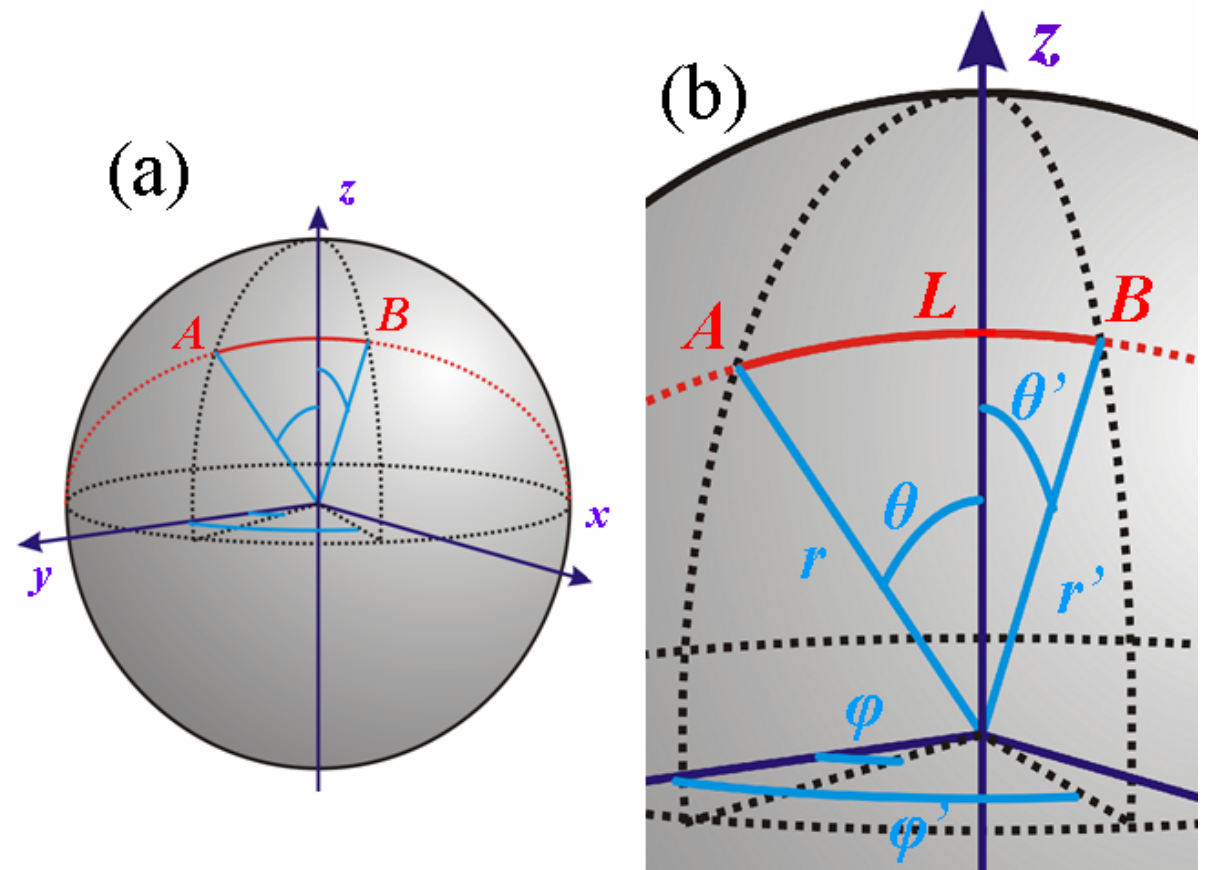

Figure 2.8.: The method employed to measure roughness takes account of the spherical topology of the grain (a), shown in detail in (b). The height difference correlation, $\rho(L)$, between pairs of points $A(r(\theta, \phi))$ and $B\left(r^{\prime}\left(\theta^{\prime}, \phi^{\prime}\right)\right)$, separated by their great-circle distance, $L$, is calculated. $R$ is the radius of the grain.

The height difference correlation, $\rho(L)$, on a double logarithmic scale, yields the vertical and lateral saturation length scales, $\xi_{v e r t}$ and $\xi_{\text {lat }}$, for a given etch time. This is the position at which a cross-over between a power-law type behaviour, with exponent $H$, and a saturation plateau occurs; figure 2.10 illustrates this.

The roughness length scales are obtained from the WLI micrographs of the spheres by the following steps: first, the WLI measurement is performed over a field-of-view of $80 \mu \mathrm{m} \times 80 \mu \mathrm{m}(60 \mu \mathrm{m} \times 60 \mu \mathrm{m}$ for the small Mo-Sci). The radius of the grain, $R$, is measured by optical micrography. The data is cropped to $60 \mu \mathrm{m} \times 60 \mu \mathrm{m}(30 \mu \mathrm{m} \times 30 \mu \mathrm{m})$ to remove the bad pixels at the edges, then filtered to remove spurious data adjacent to known bad pixel sites. Bad pixels and their neighbours make up less than $1 \%$ of the cropped data. Next, the measured $R$ and an estimate of the $x$ and $y$ positions of the "north pole" are taken as start values for a hemispherical fit to the data (figure 2.11). Using the position of the centre of the grain, $x_{0}, y_{0}$ and $z_{0}$, obtained from the hemispherical fit, the data is converted to spherical polar coordinates $r, \theta$ and $\phi$.

Following this, $\rho(L)$ is calculated, taking account of the angle-of-sight correction. Three million randomly-chosen pairs of points are taken to calculate $\rho(L)$ for each grain. $\xi_{\text {vert }}$ is obtained by laying a spline through $\rho(L)$; the length $L_{k i n k}$ 


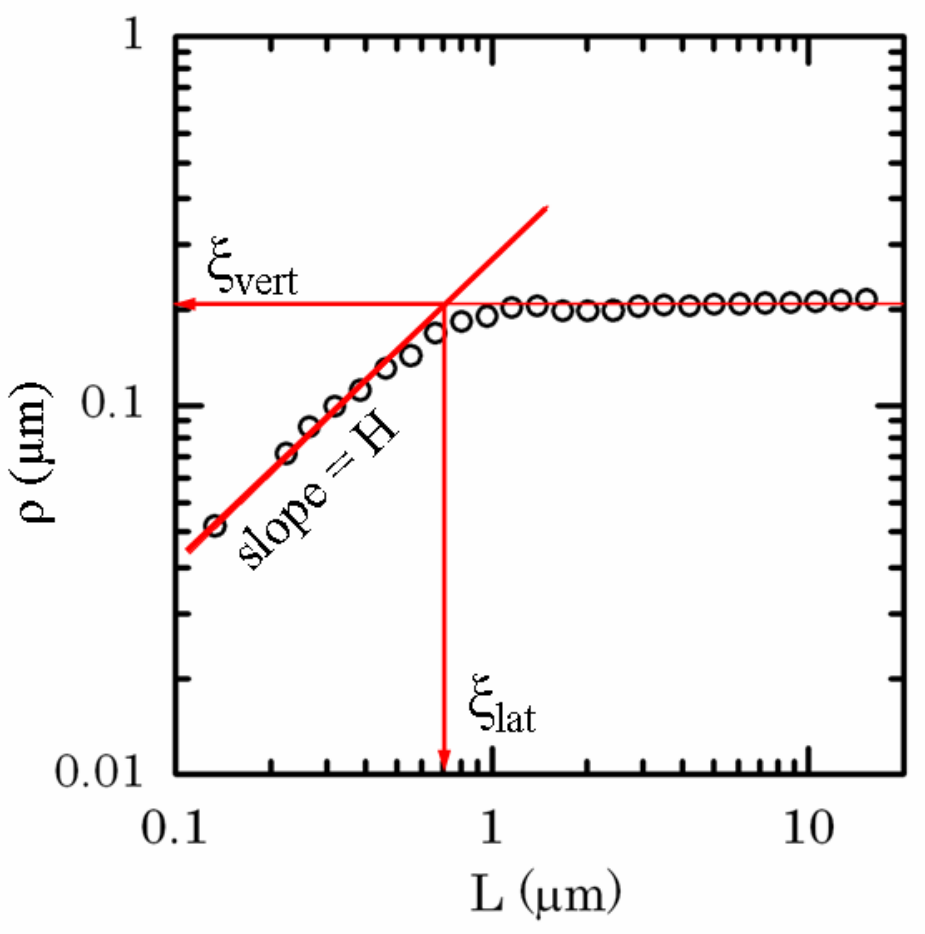

Figure 2.10.: The roughness, $\rho(L)$, on a double logarithmic scale, yields the vertical and lateral saturation length scales, $\xi_{\text {vert }}$ and $\xi_{\text {lat }}$, here for a Cataphote grain etched for 30 seconds. The slope of the fit through the first five data points is the Hurst exponent, $H$.

at which the slope of this spline becomes zero is detected. $\rho(L)$ is then averaged for $L>L_{k i n k}$, giving $\xi_{\text {vert }}$. The slope of a linear fit through the first five ${ }^{6}$ data points plotted on a double logarithmic scale gives $H$. The cross-over of the linear fit and the plateau $\left(\xi_{\text {vert }}\right)$ gives $\xi_{\text {lat }}$.

\subsubsection{Radial averaging to test for aspherical grains}

Strong asphericity in the grain will lead to distortion of the roughness analysis, since our analysis assumes a spherical topology. To establish the quality of the data, radial averaging is performed (equation 2.7) and data from aspherical grains is discarded.

$$
\left.\langle\Delta r(\theta)\rangle\right|_{\text {all } \phi} \text {. }
$$

For a perfect sphere, both the radial mean and the standard deviation are zero for all $\theta$. For rough grains, small deviations from zero are expected; for aspherical

\footnotetext{
${ }^{6}$ Five is a compromise between having as many data points as possible for the fit without fitting to points at the kink.
} 


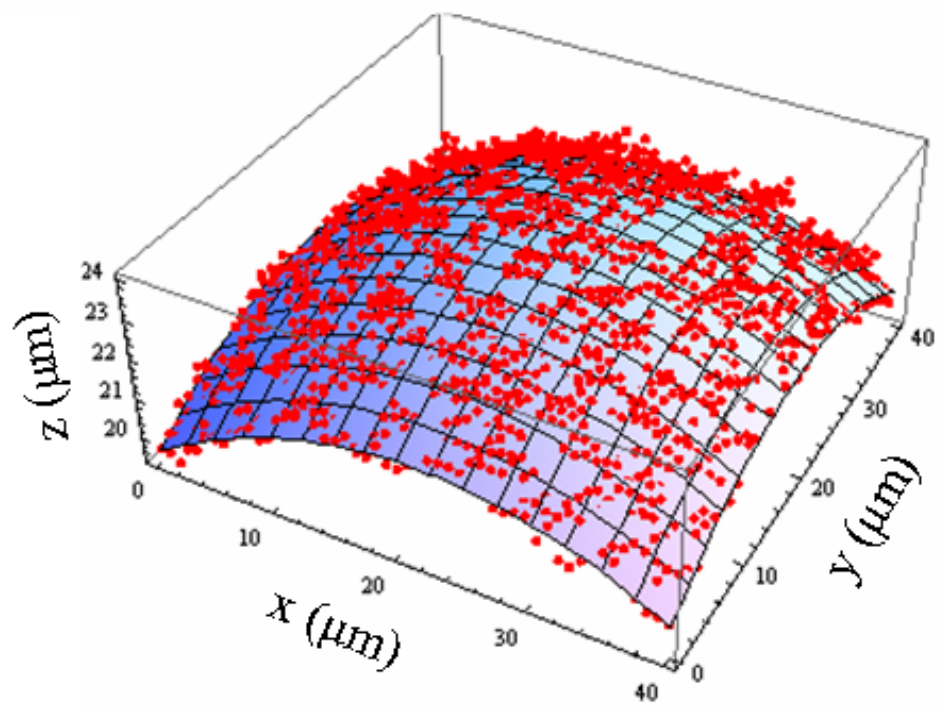

Figure 2.11.: A hemispherical fit is performed to find the values of $x_{0}, y_{0}$ and $z_{0}$, allowing us to convert our data to spherical polar coordinates. To save computing time, 100th of the WLI data points (the red dots) is used for the fit.

grains large deviations. Since our analysis depends on our ability to locate the kink in $\rho(L)$, it is vital that the mean and the standard deviation are smaller than the expectation values of the length scales we wish to measure, $\xi_{\text {vert }}$ and $\xi_{\text {lat }}$. We set the criterion that, if the radial mean OR standard deviation is greater than $\xi_{v e r t}$ for any angle corresponding to a radial distance $S<5 \xi_{\text {lat }}$, we reject the grain from our analysis, on the grounds that its asphericity has an influence on its $\rho(L)$ at the relevant length scales. This is illustrated in figure 2.12 .

Of the fifteen grains measured per batch, on average two were rejected for asphericity.

\subsection{Roughness results}

The effect of etching on the topography of glass grains is presented in this section. We discuss the vertical and lateral roughness length scales and the Hurst exponent as a function of etch time.

\section{Smoothed grains}

The grains smoothed with $\mathrm{NaOH}$ showed no difference in their roughness to the unetched grains within the error. Figure 2.13 allows us to make a visual comparison of the unetched and the smoothed glass spheres after they have been sputter coated with a $\sim 40 \mathrm{~nm}$ layer of gold. We can see that the roughness of the unetched surface (right) is caused by small flakes or crumbs that have become stuck 

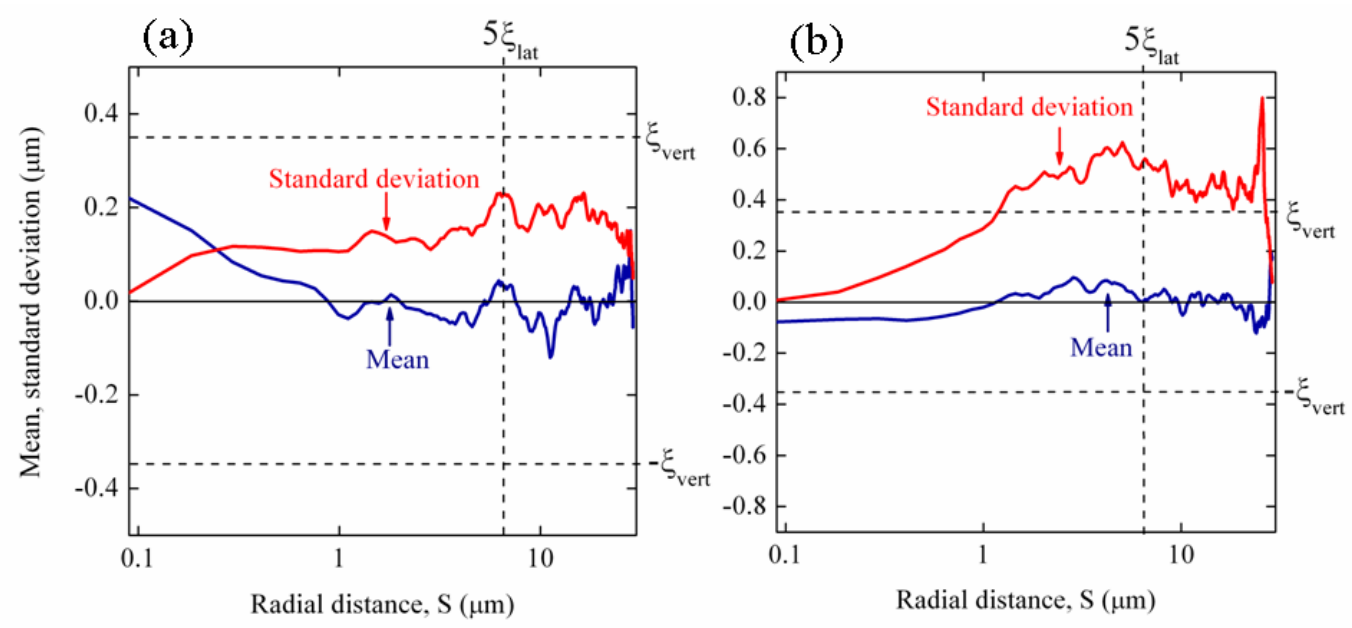

Figure 2.12.: (a) Radial average (blue) and standard deviation (red) of a large Mo-Sci grain etched for one minute. This grain is accepted: both remain within the range $\left[-\xi_{\text {vert }} \ldots \xi_{\text {vert }}\right]$ for $S<5 \xi_{\text {lat }}$. (b) Radial average and standard deviation of a different large Mo-Sci grain etched for one minute. This grain is rejected: the standard deviation exceeds the range $\left[-\xi_{\text {vert }} \ldots \xi_{\text {vert }}\right]$ for $S<5 \xi_{\text {lat }}$.

to the surface during the manufacturing of the spheres. Atomic force microscopy on uncoated grains shows that these flakes are of the same material as the rest of the surface 7 Since these flakes are rather sparse, only a handful of them appear within the region of interest used in the roughness analysis. Correspondingly small is therefore their contribution to the statistical roughness analysed using the height-difference correlation function. The smoothed grain (left) reveals the nature of the smoothing protocol: the surface as a whole has not been attacked by the etching agent, only the flakes stuck to the surface have been reduced in size. Again, their sparseness makes them virtually invisible to the roughness analysis.

\section{Roughened grains}

The effect of etching in $\mathrm{NH}_{4} \mathrm{FHF}$ on the topography of glass grains is shown in figures 2.14, 2.15 and 2.16, where the mean lateral and vertical roughness length scales and the Hurst exponent are plotted as a function of etch time. The error bars are the standard deviation; for each grain type ten to fifteen grains were averaged. The grains smoothed with $\mathrm{NaOH}$ have been omitted from the graphs, since all three of their roughness features were indistinguishable from those of the unetched grains.

\footnotetext{
${ }^{7}$ In tapping mode AFM, the phase lag of the cantilever oscillation is sensitive to variations in material properties such as surface adhesion and hardness. Our phase-imaging microscopy indicates that the surface of the grains is of the same material. Atomic force microscopy is discussed in section 2.4
} 


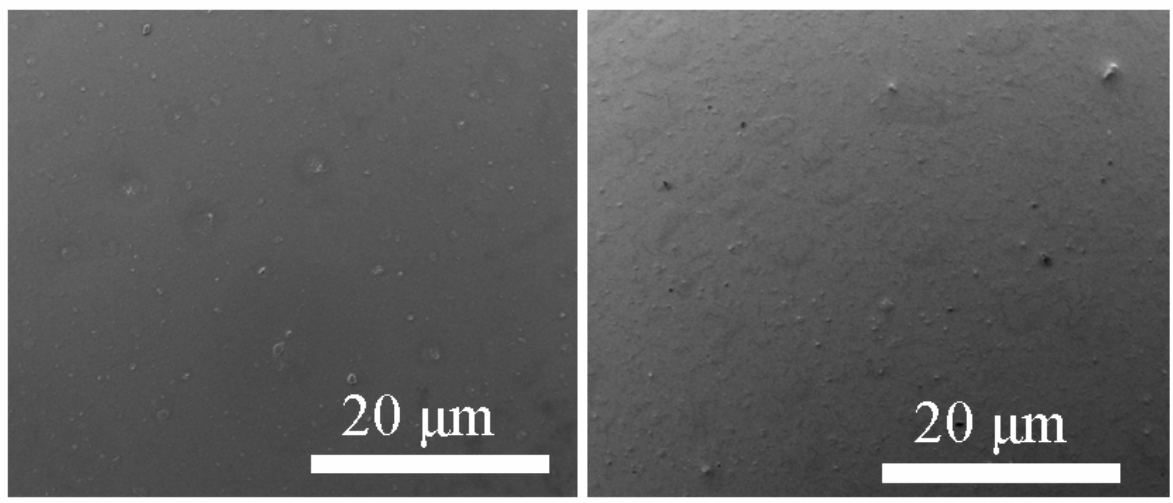

Figure 2.13.: Scanning electron microscopy (SEM) images of the surfaces of small MoSci glass spheres. Left: smoothed by NaOH. Right: unetched. Courtesy of K. Sand, University of Copenhagen.

Let us first look at the lateral roughness length scale, $\xi_{\text {lat }}$, in figure 2.14. This describes the width of surface roughness features as a function of exposure to $\mathrm{NH}_{4} \mathrm{FHF}$. For all three types of grains, we see a monotonic increase in $\xi_{\text {lat }}$ as a function of etch time, but the rate is different in each case. The small Mo-Sci particles have the highest rate of roughening: a linear fit through the data yields an estimated rate of $1.40 \mu \mathrm{m} /$ minute, although, of course, there is no reason to assume that the trend is linear. The $\xi_{\text {lat }}$ of the large Mo-Sci grains increased at a rate of $0.7 \mu \mathrm{m} /$ minute; and that of the Cataphote grains at a rate of 0.2 $\mu \mathrm{m} /$ minute. The different rates of roughening confirm the necessity of testing several types of grains: even the two types of Mo-Sci grains, which were made in the same factory, by the same process and using the same raw material 8 showed different etch rates. However, all three grain types showed the monotonic increase in $\xi_{\text {lat }}$ for which we had been aiming.

Something different is happening to the depth of the surface features, $\xi_{v e r t}$, as the etch time increases (figure 2.15). The small Mo-Sci grains again show a well-behaved monotonic increase in feature size, with $\xi_{\text {vert }}$ increasing at a rate of $0.17 \mu \mathrm{m} /$ minute. This behaviour is, again, what we would expect of a chemical etching procedure. But the two larger types of grains - the large Mo-Sci and the Cataphote - show that $\xi_{\text {vert }}$ increases in a step function. Here, we can only distinguish between unetched and etched grains: the amount of time they spent in the etching solution seems to be irrelevant at these time scales. The mean feature depth of the etched Cataphote grains is half of that of the large Mo-Sci grains.

Figures 2.15 and 2.14 show us that not only is the speed of the etching reaction different for the three types of grains, but the type of roughening can be qualitatively different. We observe two types of roughening behaviour: a monotonic

\footnotetext{
${ }^{8}$ Recycled car windscreens
} 


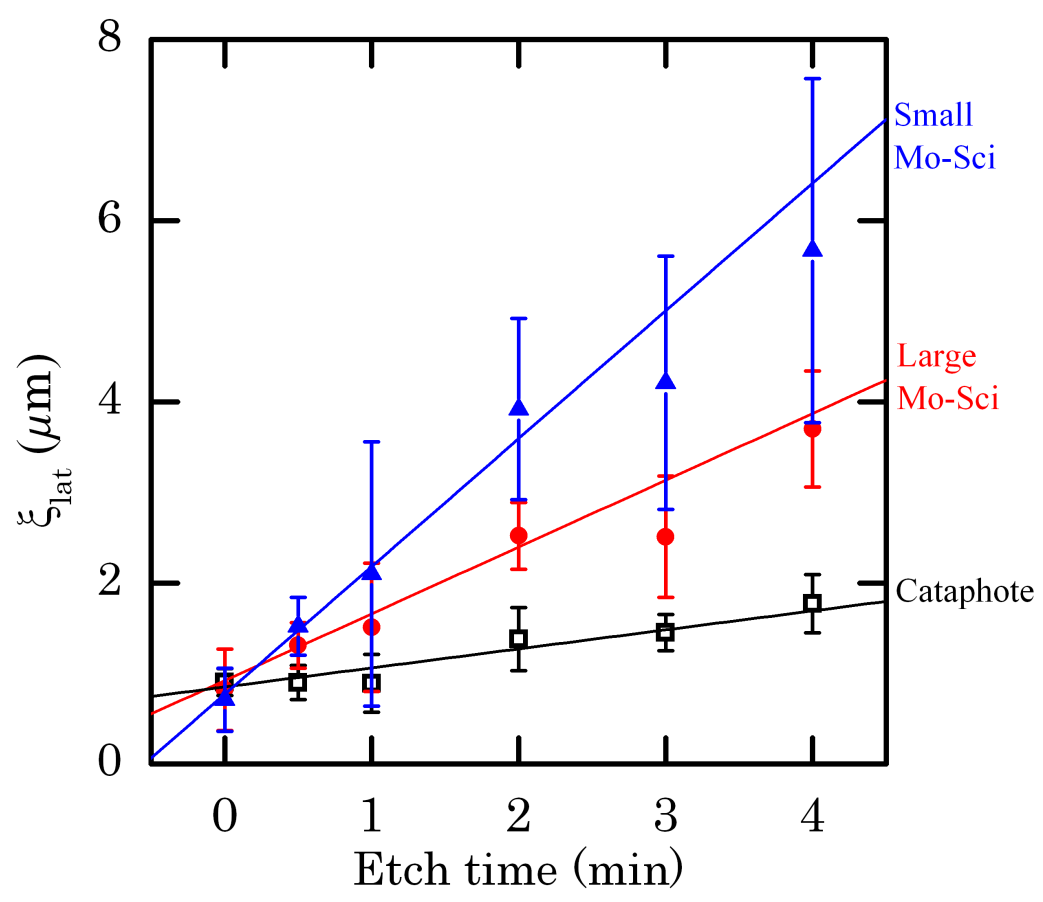

Figure 2.14.: The mean lateral roughness length scale as a function of roughening etch time. The symbols correspond to table 2.1. In this and all subsequent plots in this chapter, the error bars are the standard deviation; for each grain type ten to fifteen grains were averaged. The lines are linear fits.

increase in feature width and depth with etch time for the small Mo-Sci grains, and a broadening behaviour for the two larger types of grains, whereby surface features become wider but not deeper as a function of etch time. What mechanism could lead to this second type of feature growth is a question that falls outside the scope of the current work, but some speculation is offered in section 6.1 .

The third number for characterising roughness that we extract from our analysis is the Hurst exponent. However, as can be seen from figure 2.10, the power-law regime of $\rho(L)$ exists over less than a single order of magnitude, so caution must be exercised in interpreting the behaviour of $H$ as a function of etch time (figure 2.16). All three grain types show a reduction in $H$ from approximately 1 to 0.5 in a trend that is the same for all three grain sets, showing that the Hurst exponent can also be tuned by the etching protocols presented here. Halsey et al. suggest that the Hurst exponent may also influence grain interactions [43; the implications of this are discussed in section 6.2. Again, the grains smoothed in $\mathrm{NaOH}$ have been omitted from the graph. 


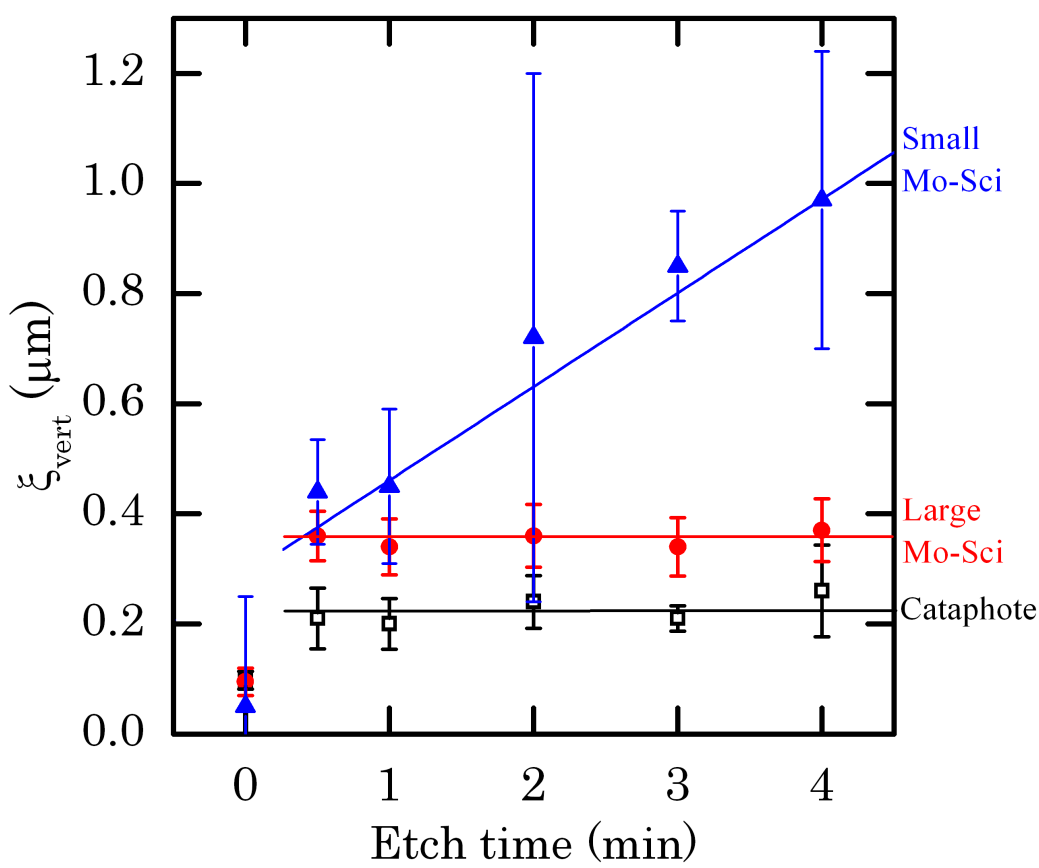

Figure 2.15.: The mean vertical roughness length scale as a function of roughening etch time. The linear fit through small Mo-Sci points and the mean $\xi_{\text {vert }}$ of the Cataphote and large Mo-Sci grains are made in the range of $30 \mathrm{~s}$ to $4 \mathrm{~min}$.

In chapter 3, we will examine how the roughness of the grain surfaces influences the bulk frictional properties of the grains. But first, we will look at two other methods of measuring the roughness of grains: atomic force microscopy and immersion in cola.

\subsection{Atomic force microscopy on grains}

As explained in section 2.2.1, when measuring the surface roughness of spheres, one is presented with a dichotomy. In order to be able to measure large correlation lengths, a large field of view is necessary, which means measuring a region in which the curvature of the grain is visible, yet in order to measure the small length scales, the measurement method must be sensitive to nano-scale features. This means that the measurement method must have a large enough dynamic range to be able to measure nano-scale features over a height range of several $\mu \mathrm{m}$.

The dynamic range of atomic force microscopy (AFM) falls short of our requirements, as illustrated in figure 2.17. Both graphs show the same $5 \mu \mathrm{m} \times$ 


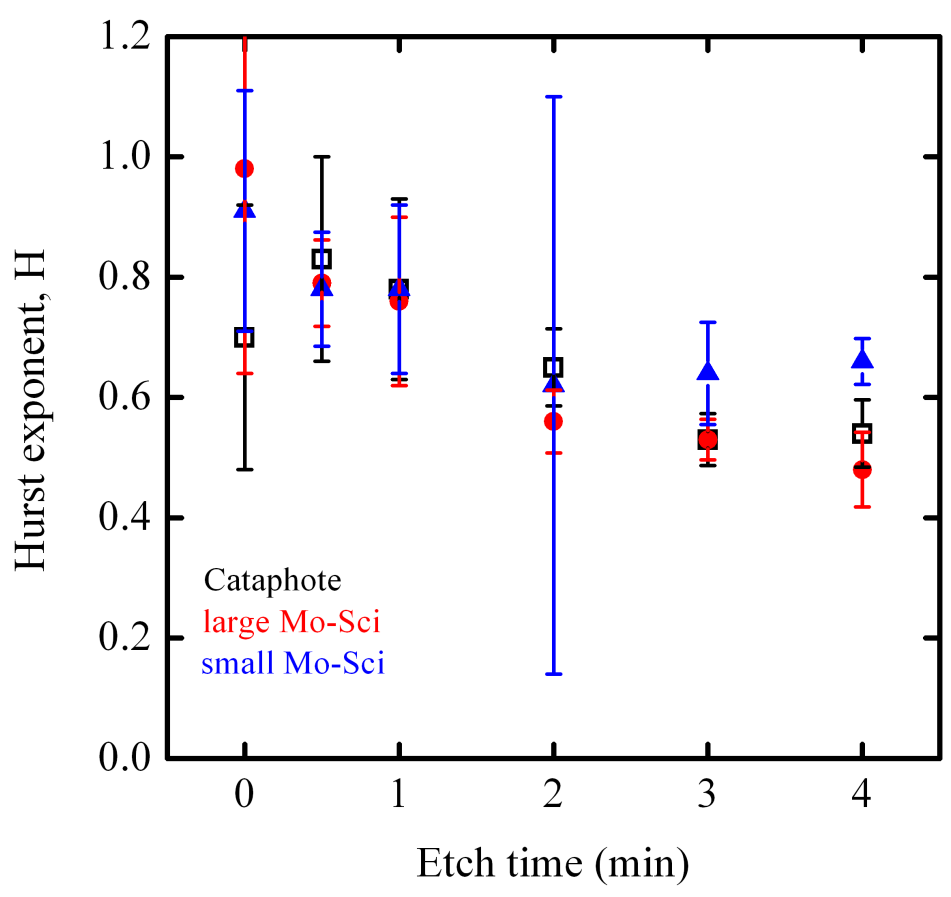

Figure 2.16.: The mean Hurst exponent as a function of roughening etch time.

$5 \mu \mathrm{m}$ region of a small Mo-Sci grain etched for four minutes; a larger region of interest exceeds the dynamic range of the cantilever. Despite our best efforts, it is impossible to guarantee that the exact top of the sphere is within the region of interest. This data set is clearly not suited to spherical fitting and has to be analysed as though there were no underlying curvature on the sample (figure 2.17(a)) or after subtraction of a second-order polynomial plane fit (figure 2.17(b)). 

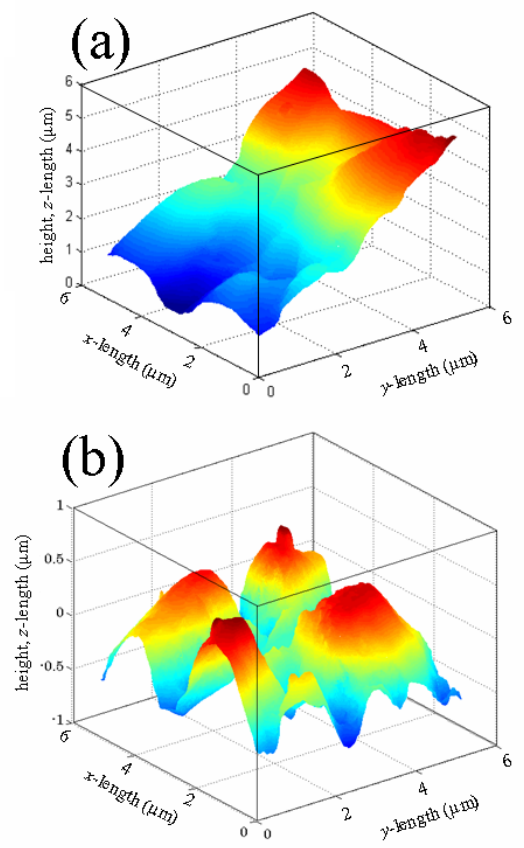

Figure 2.17.: (a) Raw AFM measurement of a small Mo-Sci grain etched for four minutes and (b) the same measurement after subtraction of a second-order polynomial plane fit. Measurement was made in tapping mode.

Neither of these approaches is satisfactory, since the first ignores the spherical topology of the grain and the second is so heavy-handed that the fit removes genuine features, especially at longer length scales. A comparison of $\rho(L)$ from these two approaches with WLI data of the same type of grain bears this out (figure 2.18): the raw AFM data yields an $\rho(L)$ without a plateau, showing that roughness information at large length scales is being dominated by the effect of the grain curvature. The AFM data with subtracted planar fit shows a kink (possibly the onset of the plateau) at misleadingly small length scales: this is because subtracting the fit has removed genuine roughness information. Moreover the $\rho(L)$ for a region of interest of $5 \mu \mathrm{m} \times 5 \mu \mathrm{m}$ is logically only available for $L \leq 5 \mu \mathrm{m}$, and we know from our WLI measurements that the length scales we wish to measure are at or above this length.

\subsection{Using a carbonated soft drink to measure the roughness of grains}

Naturally, it would be nice to have a faster and easier way of measuring the surface roughness of grains. The popular experiment in which a "geyser" is produced by dropping Mentos into a bottle of Diet Coke [55] was studied systematically by 


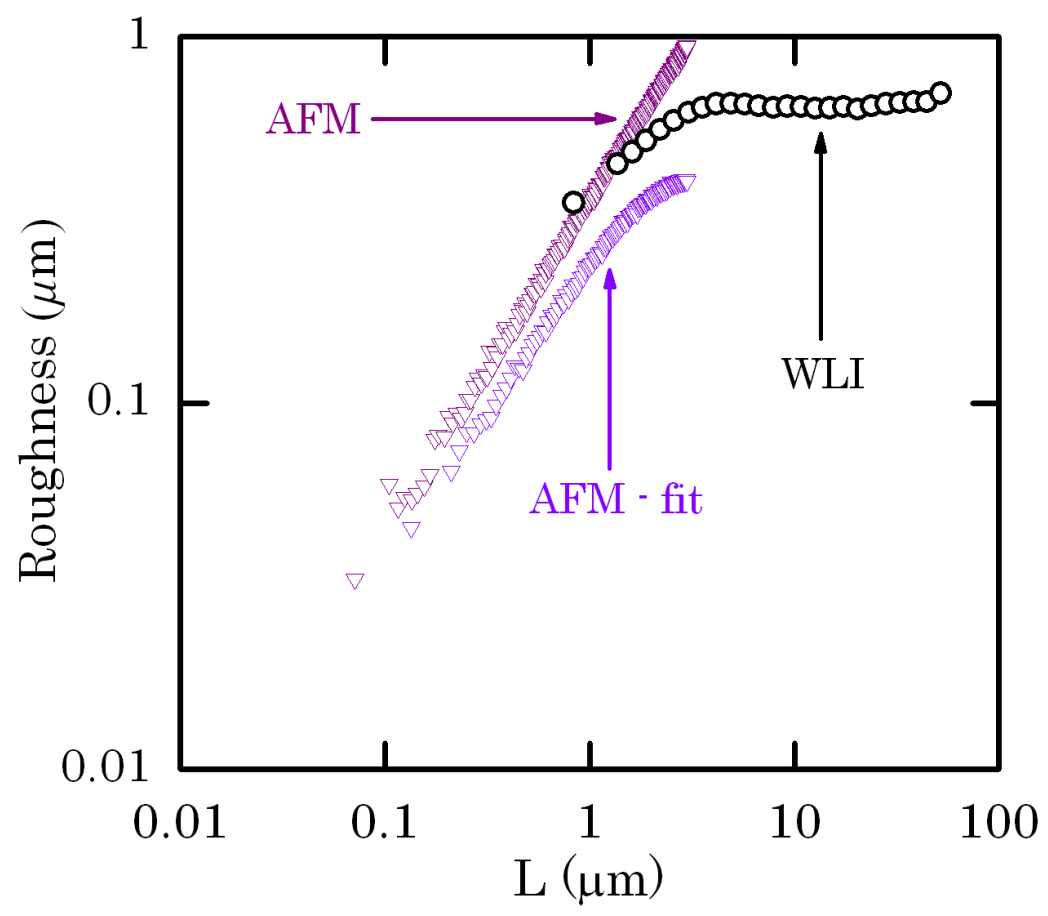

Figure 2.18.: The roughness, $\rho(L)$, for small Mo-Sci grains etched for four minutes. Comparison of two measuring techniques: AFM (triangles) and WLI (circles). The AFM data are shown raw (burgundy) and after subtraction of a second order polynomial plane fit (purple).

Coffey [56]. She concluded that the surface roughness of the sweets is one of the main causes of the reaction; namely, surface features provide nucleation sites for bubbles of carbon dioxide. We use this principle in a prototype experiment for measuring surface roughness in bulk granular samples.

The experimental method is as follows: $4 \mathrm{ml} \pm 0.05 \mathrm{ml}$ of a freshly-opened carbonated soft drink (Coca-Cola) is poured into a measuring cylinder. Pouring is smooth to minimise premature nucleation of bubbles. $500 \mathrm{mg} \pm 0.5 \mathrm{mg}$ of granular sample (small Mo-Sci) is added through a funnel. The maximum volume of cola, beads and foam is measured and the initial volume of cola subtracted.

Figure 2.19 shows that there is a clear trend: the cola produces more foam when it comes into contact with the more strongly etched particles. Of course, not just surface roughness but also other effects, such as surface activity, buoyancy and bubble occupation density of nucleation sites play a vital role in the reaction [56]. It is interesting to speculate on how this method could be calibrated. 


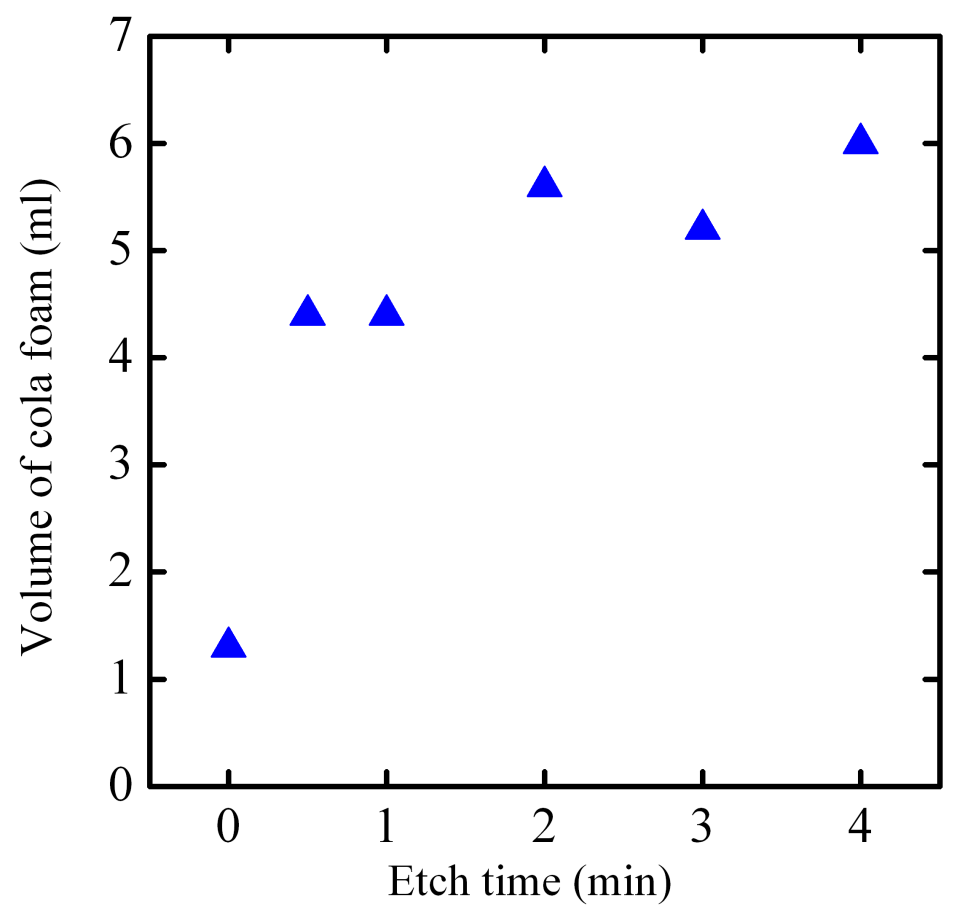

Figure 2.19.: Maximum volume of cola foam as a function of etch time (small Mo-Sci; smoothed grains were not measured).

\subsection{Conclusion}

In this chapter, we looked at modifying the roughness of soda-lime glass spheres, with the aim of tuning the inter-particle friction.

A chemical etching protocol based on ammonium bifluoride was developed to increase the roughness of glass spheres. We showed that this protocol etches more homogeneously than hydrofluoric acid; furthermore, it optimises the flow properties of the etch solution whilst releasing as little hydrofluoric acid as possible. A gentle etching solution of dilute sodium hydroxide was used to smoothen the grains (section 2.1). In section 2.1.1, the effect of etching on particle size was shown to be negligible.

Two microscopic methods were used to measure the roughness of the grain surfaces: atomic force microscopy (AFM) and white light interferometry (WLI). For our purposes, WLI is by far the better method because of the dynamic range it offers (section 2.4).

A method of statistical roughness analysis, based on the height-difference correlation function of reference [52], was adapted to measure roughness on a spherical 
surface, taking account of the angle of sight of the WLI measurement (section 2.2.1). However, this method gives false results for aspherical grains, so a test for grain asphericity was also made (section 2.2.2). The roughness analysis yields a vertical and a lateral roughness length scale $\left(\xi_{\text {vert }}\right.$ and $\left.\xi_{\text {lat }}\right)$ and the Hurst exponent, $H$.

It was shown that the roughening protocol enables us to tune the roughness of soda-lime glass spheres, albeit in not in a straightforward way (section 2.3). One type of grain showed a monotonic increase in $\xi_{v e r t}$ and $\xi_{l a t}$ as a function of roughening etch time. The two other types we tested showed a steady increase in $\xi_{\text {lat }}$, but a constant $\xi_{\text {vert }}$ for all roughening etch times. All three grain types showed a reduction in $H$ with etching from approximately 1 to 0.5 . The apparent smoothing effect of the $\mathrm{NaOH}$ seen in the SEM images of figure 2.13 was not borne out by the roughness analysis.

A method for measuring the roughness of bulk samples was suggested in section 2.5. Here, the nucleation of bubbles in a granular sample immersed in cola was shown to be an indicator of grain roughness.

Now we have seen that it is possible to modify the roughness of glass grains, we need to look at if and how this has changed the inter-particle friction. This is the subject of the following chapter. 



\section{Bulk friction}

In this chapter, the angle of repose, $\alpha_{r}$, is measured in order to characterise the bulk frictional behaviour of our grains. This measure for friction is verified by shear measurements of the angle of internal friction in stationary flow, $\alpha_{s f}$. The effects of grain asphericity, $a$, and volume fraction, $\phi$, on the stability of granular piles are investigated.

\subsection{Angle of repose}

In essence, the experiment is the same as the hourglass we discussed in the introduction. Grains rain through an aperture onto a pile. A growing granular pile has an angle $\alpha_{r} \leq \alpha \leq \alpha_{m s}$ to the horizontal, where $\alpha_{r}$ is the angle of repose and $\alpha_{m s}$ the angle of maximum stability. The grains that land on the top of the pile increase the embankment angle $\alpha$ and intermittent avalanches reduce it. Figure 3.1, taken from Courrech du Pont et al. (2003) shows the time evolution of $\alpha$ (called $\theta$ in the figure) for a rotating drum experiment.

\section{A few notes of caution}

The idea that a slope grows until it has reached a global angle $\alpha_{m s}$, then avalanches until it has reached $\alpha_{r}$ is only true after large avalanches in which the whole surface has taken part in the avalanche. Smaller avalanches have the effect of roughening the surface of the pile so that no single $\alpha$ can be used to describe the pile. One can only know where in the range $\alpha_{r} \leq \alpha \leq \alpha_{m s}$ the pile is the instant a whole-surface avalanche starts $\left(\alpha=\alpha_{m s}\right)$ or the moment it stops $\left(\alpha=\alpha_{r}\right)$.

This description only applies in a quasi-static flow regime. The quasi-static realisation of our experiment would mean adding a single grain, observing the pile, waiting for any motion to stop, then adding another grain; and so on. A slow experiment indeed. The experiment we perform is not in the quasi-static regime. The intermittent dynamics and the embankment angles we see are disguised and influenced by the constant stream of grains: here, it is possible to exceed $\alpha_{m s}$ simply because one is adding grains to the top of the pile faster than they can flow away. $\alpha_{m s}$ is therefore inaccessible to us in this experiment, so we measure $\alpha_{r}$, but even here, we need to make sure we do not distort the measurement by adding more grains to the pile before an avalanche has finished. 


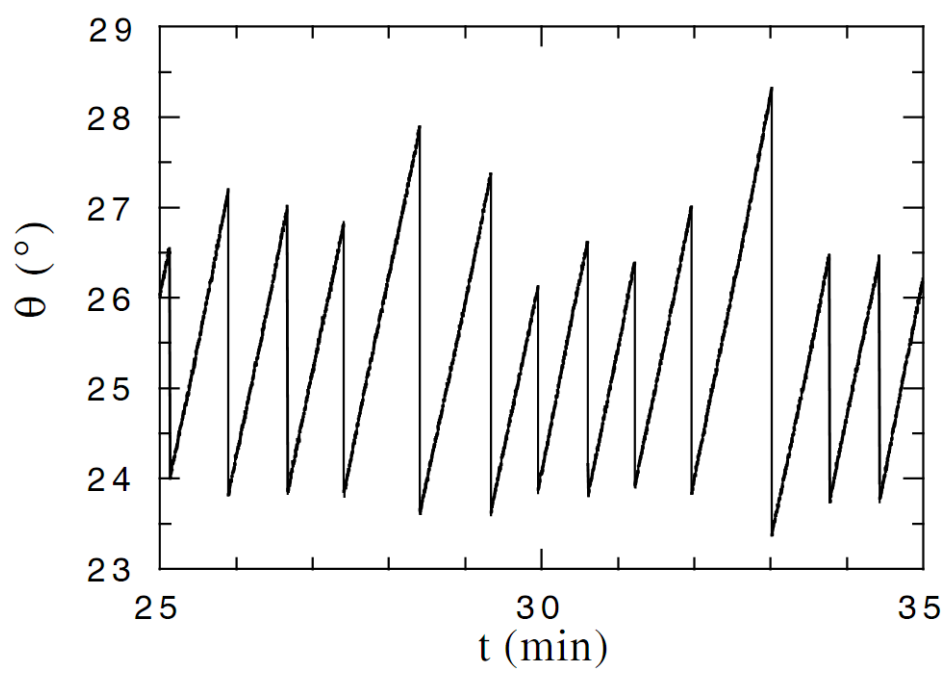

Figure 3.1.: The embankment angle as a function of time in a slowly rotating drum half filled with glass spheres $230 \mu \mathrm{m} \pm 30 \mu \mathrm{m}$ in diameter. The slope increases slowly as the drum rotates and the decreases rapidly when an avalanche occurs. $\alpha_{r}$ is about $24^{\circ}, \alpha_{m s}$ is around 26.5 . Figure taken from [57]. A similar time evolution would be expected for the sedimenting granular pile of our experiment (figure 3.3), but in this case it is the addition of grains to the top of the pile that is causing the slope to increase.

Our method is as follows: we add grains to the pile in a narrow stream at a rate of $\sim 140$ grains per second. Once the entire surface of the pile is flowing - a system-spanning avalanche - we stop the addition of grains. The avalanche continues for twenty to 30 seconds, after which the pile comes to rest. The angle at which the pile comes to rest is $\alpha_{r}$.

It is also important to note the boundary conditions of the pile. As we saw in figure 1.5, the forces in a granular pile depend on how the pile was made. Since the force network determines the stability of a granular pile, it also determines $\alpha_{r}$. Figure 3.2 shows three possible pile configurations, each with a different $\alpha_{r}$. There is no reason to suppose that any one boundary configuration is superior. We use the free foot boundary condition (which corresponds to figure $3.2(\mathrm{~b})$ ).

\section{Experimental set-up}

The angle of repose of each of the 21 samples is measured using the set-up described in figure 3.3. To eliminate electrostatic and capillary attraction between grains, the measurements are made under water. A cell $25 \mathrm{~cm}$ wide, $30 \mathrm{~cm}$ high and 40 grain diameters deep is filled with water $1^{1}$ The water temperature is kept

\footnotetext{
${ }^{1} 1 \mathrm{~cm}$ for large Mo-Sci and Cataphote, $0.5 \mathrm{~cm}$ for small Mo-Sci. The width of the cell is a compromise. In order to reduce the relative effect of the walls, it should be wide, but if avalanches are to leave a flat pile surface, it needs to be narrow. For spherical particles with
} 

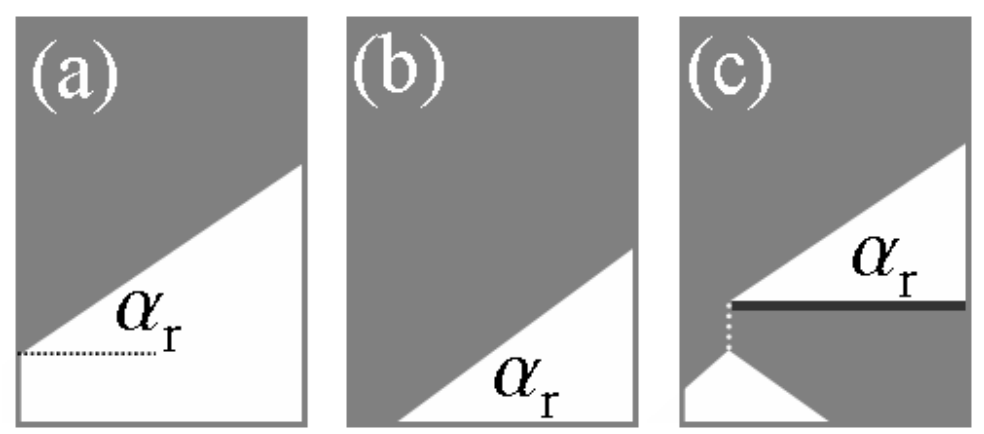

Figure 3.2.: Three possible boundary conditions for a growing granular pile: (a) foot of the pile is blocked ("blocked foot"); (b) foot of the pile is free ("free foot"); (c) pile on a platform ("no foot"). $\alpha_{r}$ is greatest in (a) and least in configuration (c). The rotating drum of [57] has a blocked foot boundary condition; our experiment has a free foot.

at $21 \pm 0.5^{\circ} \mathrm{C}$ for all experiments to keep the viscosity constant.

An upper reservoir is filled with about $30 \mathrm{~g}$ of the granular sample. A rod can be raised on a screw thread to allow the grains to flow to the bottom part of the cell at a rate of approximately 140 grains/s. When the whole of the pile surface is moving, the flow of grains to the pile is stopped by lowering the rod. The pile is photographed with a black-and-white CCD with 1 Megapixel and 8-bit depth. In order to make sure that each measurement has the same accuracy, the pile is first allowed to grow until it spans the entire camera frame $(\sim 8 \mathrm{~cm})$. Each photograph is binarised using a threshold that is chosen automatically to fall between the peaks of its bimodal grey-level histogram. The top and bottom edges of the pile are detected, and linear fits are made to the top and bottom edges of the pile; the angle between them is the angle of repose, $\alpha_{r}$. The measurement is repeated ten to twenty times per grain type. The resolution in $\alpha_{r}$ is $\pm 0.15^{\circ}$.

\subsubsection{Results}

Figure 3.4 shows $\alpha_{r}$ as a function of etch time. Each point is the mean of ten to twenty measurements and the error bar is the standard deviation. For the small Mo-Sci grains, the angle of repose increases monotonically from about $24^{\circ}$ to over $27^{\circ}$ as a function of etch time. A greater $\alpha_{r}$ indicates a greater inter-particle friction, $\mu$. We use Coulomb's definition for granular friction [10], 2] whereby

a fairly low friction coefficient, the influence of the side walls can be calculated and has been measured experimentally [58, 59. For smooth glass beads and smooth side walls, 40 grain diameters are enough to allow the effect of the walls to be ignored [58. The calculations can be found in appendix $\mathrm{A}$

${ }^{2}$ Coulomb did not stipulate boundary conditions (figure 3.2 , so we are free to use this definition for our $\alpha_{r}$. 


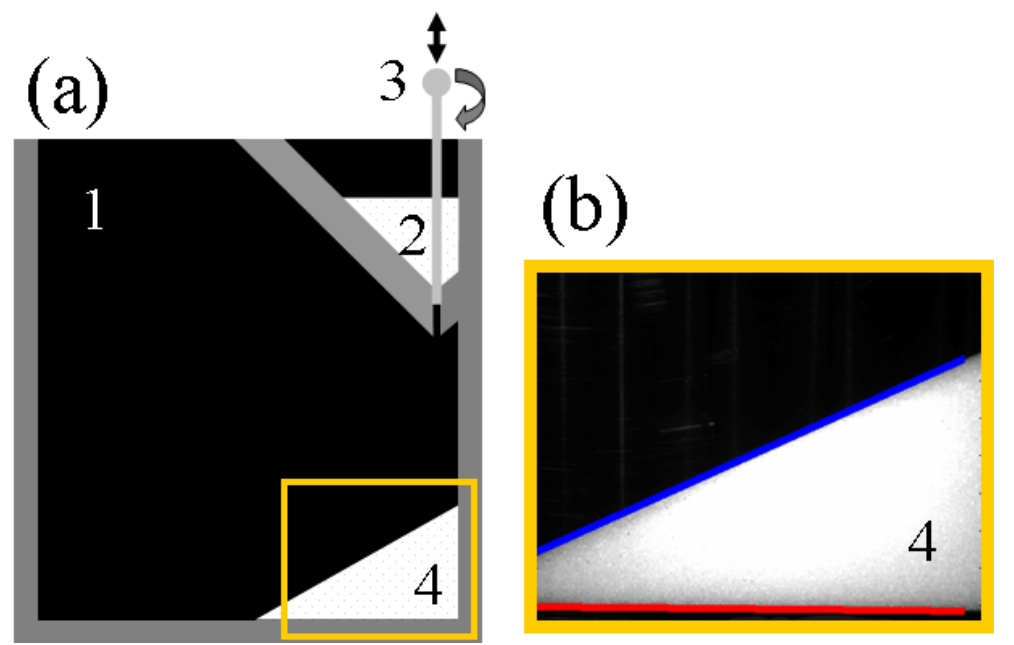

Figure 3.3.: Angle of repose measurement set-up. (a) A sketch of the set-up: a cell [1] $25 \mathrm{~cm}$ wide, $30 \mathrm{~cm}$ high and 40 grain diameters deep is filled with water. The reservoir [2] is filled with the granular sample. The funnel rod [3] is raised by a screw thread to open the hole enough to let grains flow to the bottom part of the cell [4]. Once the whole surface of the pile is flowing, the rod is lowered enough to induce granular arching, thus stopping the flow of grains. (b) CCD image of the pile. The region of interest, shown in yellow, is approximately $8 \times 6 \mathrm{~cm}$. Linear fits to the bottom (red line) and top edges (blue) of the pile allow us to calculate the angle of repose.

$$
\mu \equiv \tan \alpha_{r}
$$

As we observed in the roughness measurements, the smoothed grains do not show an appreciable difference to the unetched grains.

The Cataphote and large Mo-Sci grains show a different behaviour to the small grains. Again, their behaviour describes a step function: unetched and smoothed grains have an angle of repose about $1^{\circ}$ less than grains roughened for $30 \mathrm{~s}$ or longer in $\mathrm{NH}_{4} \mathrm{FHF}$. This trend looks similar to $\xi_{\text {vert }}$ as a function of etch time shown in figure 2.15; this might suggest that it is the vertical length scale that determines the frictional properties of grains. However, the Cataphote grains, whose $\xi_{\text {vert }}$ was always smaller than the large Mo-Sci grains, have a consistently larger angle of repose. This discrepancy is discussed in section 3.2.1

The main goal of the work in this and the previous chapter is to see how the frictional properties of grains depend on their (tailored) roughness. This result is shown in figure 3.5: the angle of repose as a function of $\xi_{\text {vert }}$ and $\xi_{\text {lat }}$. The angle of repose shows no appreciable correlation with $\xi_{\text {lat }}$ but a weak/noisy one with $\xi_{\text {vert }}$, again suggesting that it is the depth of surface features, not their width, that controls the friction between grains. However, it is not obvious what mechanism could lead to this behaviour, and there is not a simple, linear dependency. 


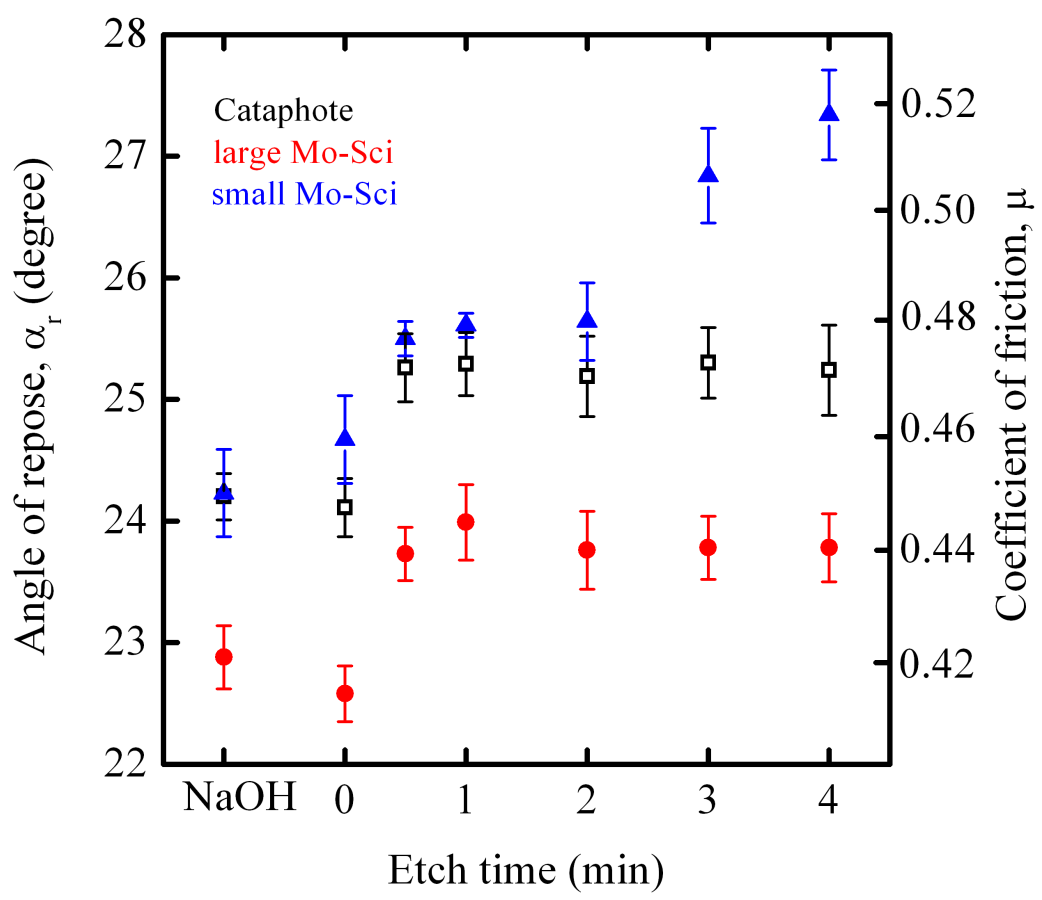

Figure 3.4.: Underwater angle of repose as a function of roughening etch time. The smoothing protocol, $\mathrm{NaOH}$, is plotted on the negative time axis. The error bars are the standard deviation of ten to fifteen measurements. The coefficient of friction is computed by $\mu \equiv \tan \alpha_{r}$.

\subsubsection{Pile relaxation time}

If we take a sequence of photographs of the pile after shutting off the flow of grains, we can observe how the angle of the pile relaxes towards $\alpha_{r}$. We did not pursue this systematically, since the flow of grains in confinement lies outside the scope of this work. Figure 3.6 shows the angle of the pile as a function of time for two types of small Mo-Sci grains: unetched and roughened for four minutes. It might be expected that higher friction leads to a more rapid arrest of the granular flow, and this is indeed what we see. Exponential fits to the data give relaxation times of $3.9 \mathrm{~s} \pm 0.5 \mathrm{~s}$ for the unetched grains and $1.4 \mathrm{~s} \pm 0.5 \mathrm{~s}$ for the grains roughened for four minutes. Although it is a less straightforward method than a direct measurement of $\alpha_{r}$, this relaxation method could probably be used to measure inter-particle friction in situations in which $\alpha_{r}$ and $\alpha_{m s}$ are inaccessible, for instance in industrial applications such as hoppers. 


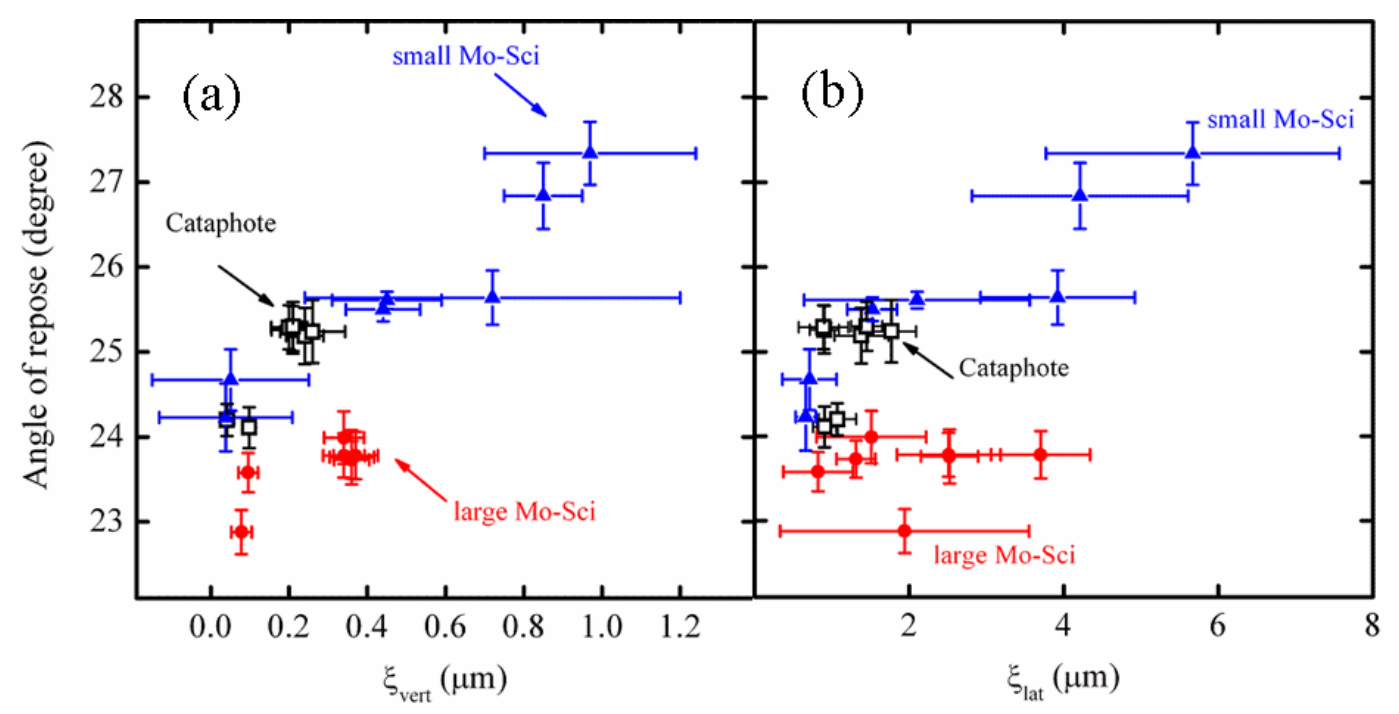

Figure 3.5.: $\quad \alpha_{r}$ as a function of roughness length scales (a) $\xi_{\text {vert }}$ and (b) $\xi_{\text {lat }}$.

\subsection{Angle of internal friction}

The angle of repose measurements are verified qualitatively using a ring shear tester RST-XS (Schwedes \& Schulze Schüttguttechnik) [60]. The ring shear tester is the current industry standard for measuring the flow properties (yield loci) of powders and granular samples. We use it to measure the angle of internal friction for stationary flow, $\alpha_{s f}$, for a given preparation protocol. Here, we describe only how $\alpha_{s f}$ is measured; the method and our results for measuring the yield locus are shown in appendix $\mathrm{B}$

The granular sample is placed into an annular shear cell, shown in figure 3.7 . The cell is equipped with vanes on the bottom and lid. The purpose of the vanes is to ensure that the grains are moved past one another in a yield plane within the sample, rather than sliding along the walls. The sample is then sheared whilst it is subjected to an arbitrary consolidation normal stress $\sigma_{\text {pre }}$. After a certain time, the shear stress, $\tau$, reaches a saturation, $\tau_{\text {pre. }}$. The sample is said to be undergoing stationary flow; this process is shown in figure 3.8. At this point, the sample is in a homogeneous and reproducible state.

The angle of internal friction at stationary flow is given by [60]

$$
\alpha_{s f}=\arctan \left(\frac{\tau_{\text {pre }}}{\sigma_{\text {pre }}}\right)
$$

$\alpha_{s f}$ is clearly of a different physical nature to $\alpha_{r}$ or $\alpha_{m s}$, but a qualitative comparison is permissible.

Figure 3.9 shows $\alpha_{s f}$ as a function of roughening etch time for our granular samples. The results are qualitatively similar to those of the angle-of-repose 


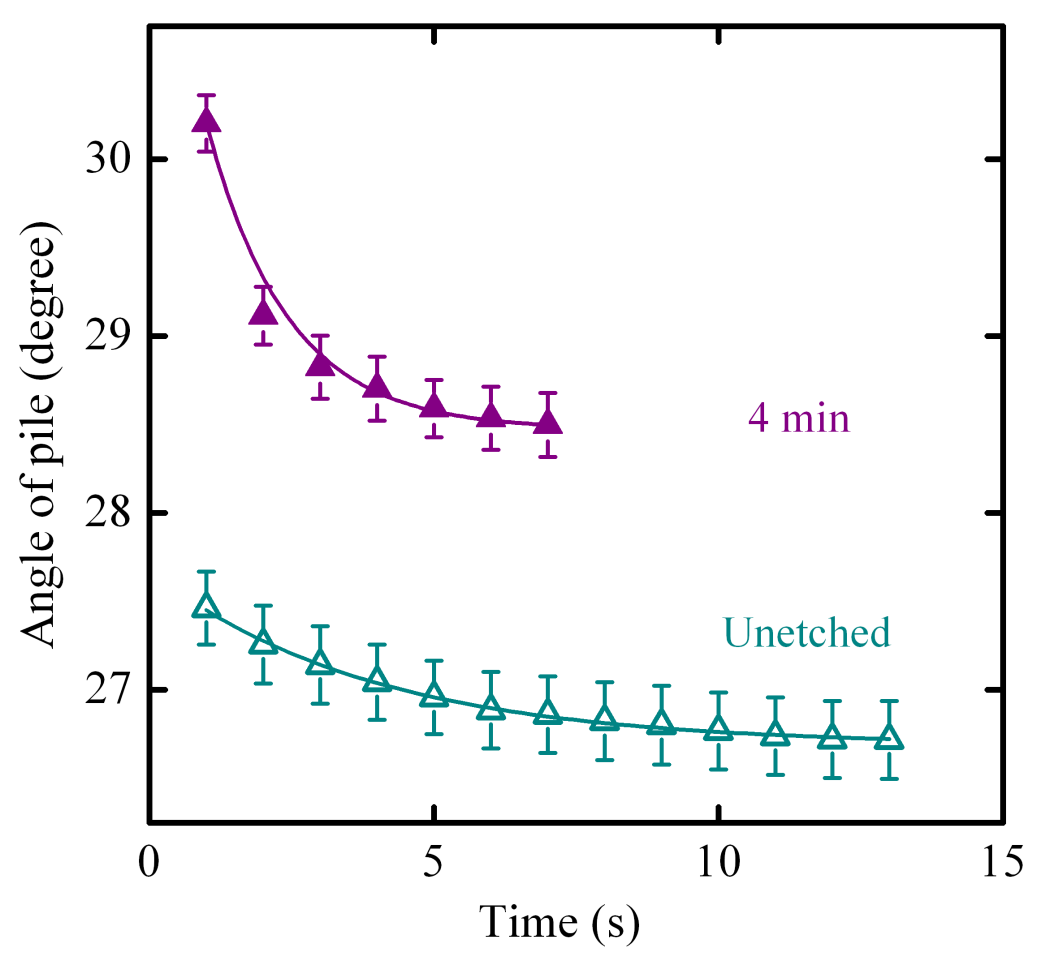

Figure 3.6.: Relaxation of the embankment angle as a function of time for unetched and roughened small Mo-Sci grains. The lines are exponential fits. Error bars are greater than the $\pm 0.15^{\circ}$ given in the description of the experiment because this data is from an earlier, less accurate realisation of the set-up.

measurement, although the Cataphote grains have a greater $\alpha_{s f}$ relative to the Mo-Sci grains than we would expect from looking at $\alpha_{r}$. We see that the Cataphote grains, whose $\xi_{\text {vert }}$ was always lower than that of the large Mo-Sci grains (figure 2.15), have a consistently greater $\alpha_{s f}$. This is a similar discrepancy to the one we saw in the $\alpha_{r}$ measurements in figure 3.4. The possible origins of this discrepancy are discussed in section 3.2.1

It seems reasonable to expect that an increase in inter-particle friction would lead to a reduction in the volume fraction, $\phi$, because frictional forces would stabilise the grains. The ring shear tester measures the height of the packing and thence estimates $\phi$ for a given $\sigma_{\text {pre }}$. Figure 3.10 shows $\phi$ as a function of roughening etch time. Despite the large error bars, we see a trend in which rough grains pack less densely than smooth when subject to the same consolidation stress. 


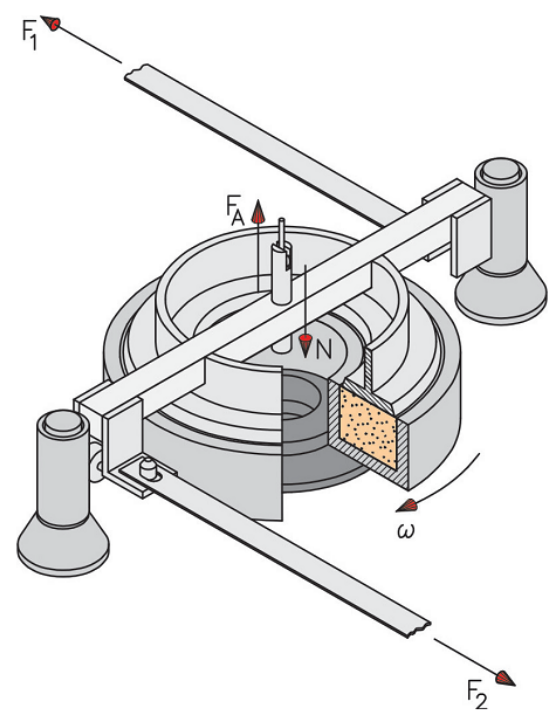

Figure 3.7.: The ring shear tester. A normal force, $N=\sigma A$, is applied to the sample via the lid, where $A$ is the sample area. A shear stress, $\tau$, is applied by rotating the container with angular velocity $\omega$ whilst the lid is held still. The shear forces, $F_{1}$ and $F_{2}$, and thence the shear stress, $\tau$, are measured via the rods holding the lid. The height of the lid is also measured, to give $\phi$. Figure taken from 60].

\subsubsection{Asphericity, contact number and volume fraction}

It has been demonstrated experimentally that higher asphericity leads to a larger angle of repose [42]. So one reason for the discrepancy between $\xi_{\text {vert }}$ and the angles $\alpha_{r}$ and $\alpha_{s f}$ of the two large types of grains could be that different fractions of non-spherical grains are present in the Cataphote and Mo-Sci samples. To pursue this hypothesis, the average length-to-width ratio of a fitted ellipse, $a=l / b$, of the unetched Cataphote and large Mo-Sci grains is measured using a Retsch Technology Camsizer (sample size of 7,000 grains); this is illustrated in figure 3.11.

With $\langle a\rangle=1.17$, the Cataphote grains show a slightly greater average asphericity than the large Mo-Sci grains, $\langle a\rangle=1.12$. There are two important mechanisms by which asphericity could affect the stability of a granular pile: by changing the number of contacts between grains, $z$, or by changing the volume fraction, $\phi$.

Figure 3.12, taken from 61, demonstrates the first of these mechanisms. An increase in $a$ from 1.12 to 1.17 leads to an increase in $z$ of $\Delta z \sim \frac{1}{2}$ for spheroids and $\Delta z \sim 1$ for ellipsoids $3^{3}$ A grain with more contacts has more constraints and will find it harder to move, which could lead to a greater $\alpha_{r}$ (or $\left.\alpha_{s f}\right)$

\footnotetext{
${ }^{3}$ The graph shows simulations of randomly packed frictionless grains. It is hard to say if it is quantitatively applicable to our measurements.
} 


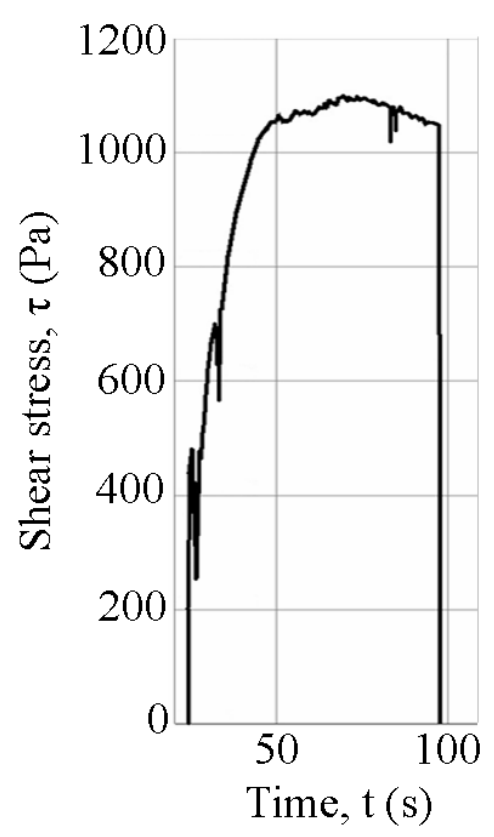

Figure 3.8.: Shear stress, $\tau$, as a function of time for a given imposed normal stress, $\sigma_{\text {pre }}$. At steady-state flow, $\tau=\tau_{\text {pre }}=$ constant. This data set is for Cataphote grains etched for one minute.

The second mechanism - the effect of asphericity on $\phi$ - is illustrated by a figure from the same reference. Figure 3.13 shows the packing densities of simulated frictionless ellipsoids. An increase in $\langle a\rangle$ from 1.12 to 1.17 would lead to an increase in $\phi$ by $1 \%$ for frictionless grains. Though we did not measure $\phi$ in the angle-of-repose set-up, we have been able to show that $\phi$ has a strong influence on the granular pile stability using a different set-up (section 3.3). There, we saw that an increase in $\phi$ by $1 \%$ leads to an increase in the angle of maximum stability by $2^{\circ}(\mathrm{NaOH})$ or $4.5^{\circ}(4$ mins $)$.

Since we do not measure how $z$ and $\phi$ change as a function of etch time, nor how (as is likely) $z$ depends on $\phi$, it is not possible for us to conclude which of these two mechanisms is responsible for the discrepancy.

\subsection{Angle of maximum stability}

We noted in the introduction that the volume fraction of a random loose packing of particles, $\phi_{R C P}$, depends on the inter-particle friction [13, 14]. By our changing the friction between grains, it seems likely that we have influenced $\phi$ in the pile of our angle of repose experiment: $\phi$ as a function of etch time measured in the shear cell (figure 3.10) certainly showed this kind of trend. It also seems reasonable to suppose that $\alpha_{r}$ depends not only on the friction between grains but also on $\phi$. 


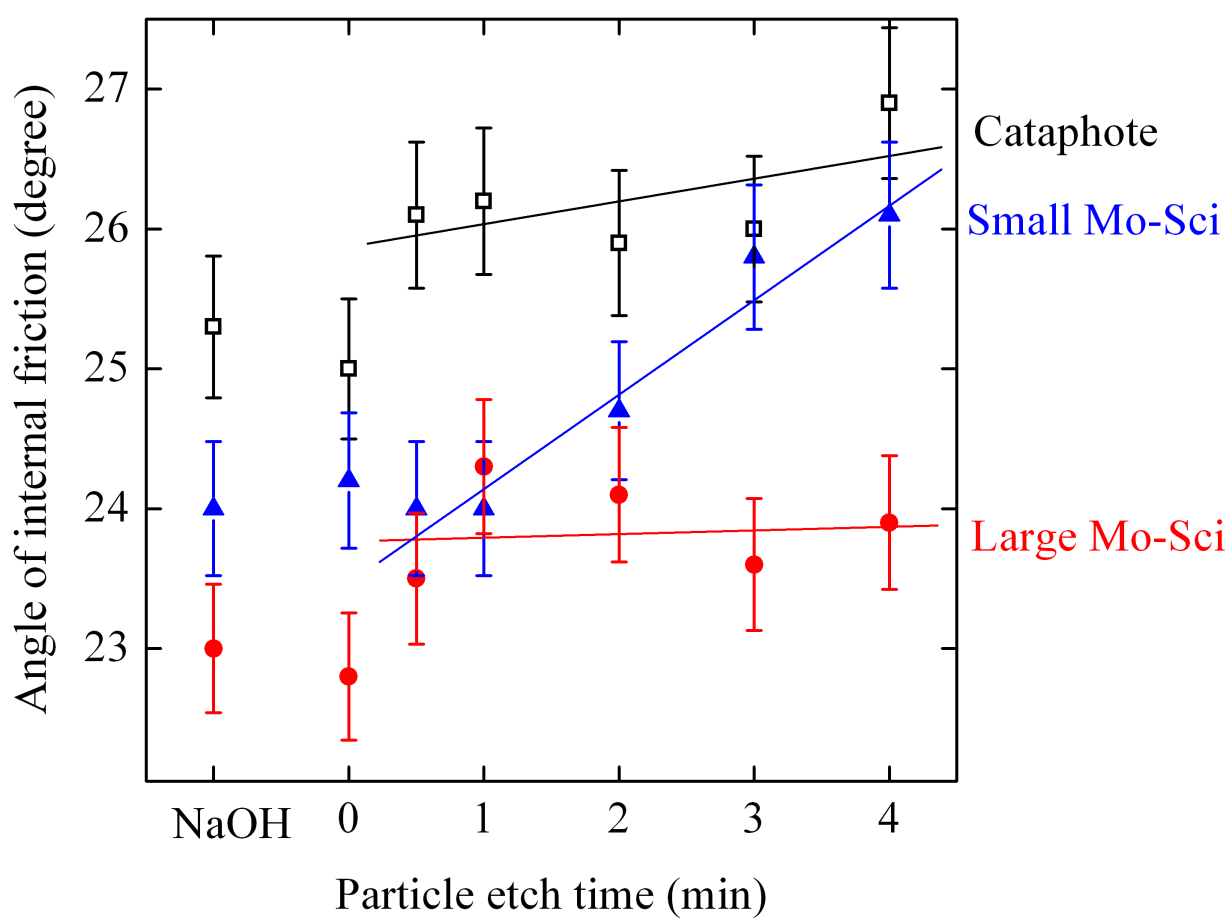

Figure 3.9.: $\quad \alpha_{s f}$ as a function of roughening etch time for all our granular samples. The linear fits through the roughened grains are merely a guide to the eye.

The same supposition can be made of the angle of maximum stability (pile slope just before an avalanche, $\alpha_{m s}$ ), which proved to be the more accessible quantity in investigating the relationship between $\phi$ and pile stability.

In this section, we investigate $\alpha_{m s}$ as a function of $\phi$. The set-up is shown in figure 3.14. A 30 g granular sample of known volume fraction is raised on a hinged support until it starts to flow/avalanche. The angle at which flow commences is $\alpha_{m s}$. Again, the experiment is performed on grains fully submersed in water. The smoothest $(\mathrm{NaOH})$ and the roughest (four minutes in $\mathrm{NH}_{4} \mathrm{FHF}$ ) samples of the small Mo-Sci grains were tested.

The experimental method is as follows. First, the granular packing is produced and $\phi$ measured. To this end, the density of the grain material is determined to within $\pm 0.45 \mu \mathrm{g} / \mathrm{cm}^{3}$ with a Micromeritics gas pyknometer. The grains are added to a cell of square cross-section $4 \mathrm{~cm} \times 4 \mathrm{~cm}$ which is filled with water. The cross-sectional area of the cell is measured as $16.51 \mathrm{~cm} \pm 0.05 \mathrm{~cm}$ by adding controlled volumes of water with an Eppendorf pipette, photographing and measuring the height of the meniscus and making a linear fit to volume vs. height. The intercept of the fit gives the systematic offset of the height 


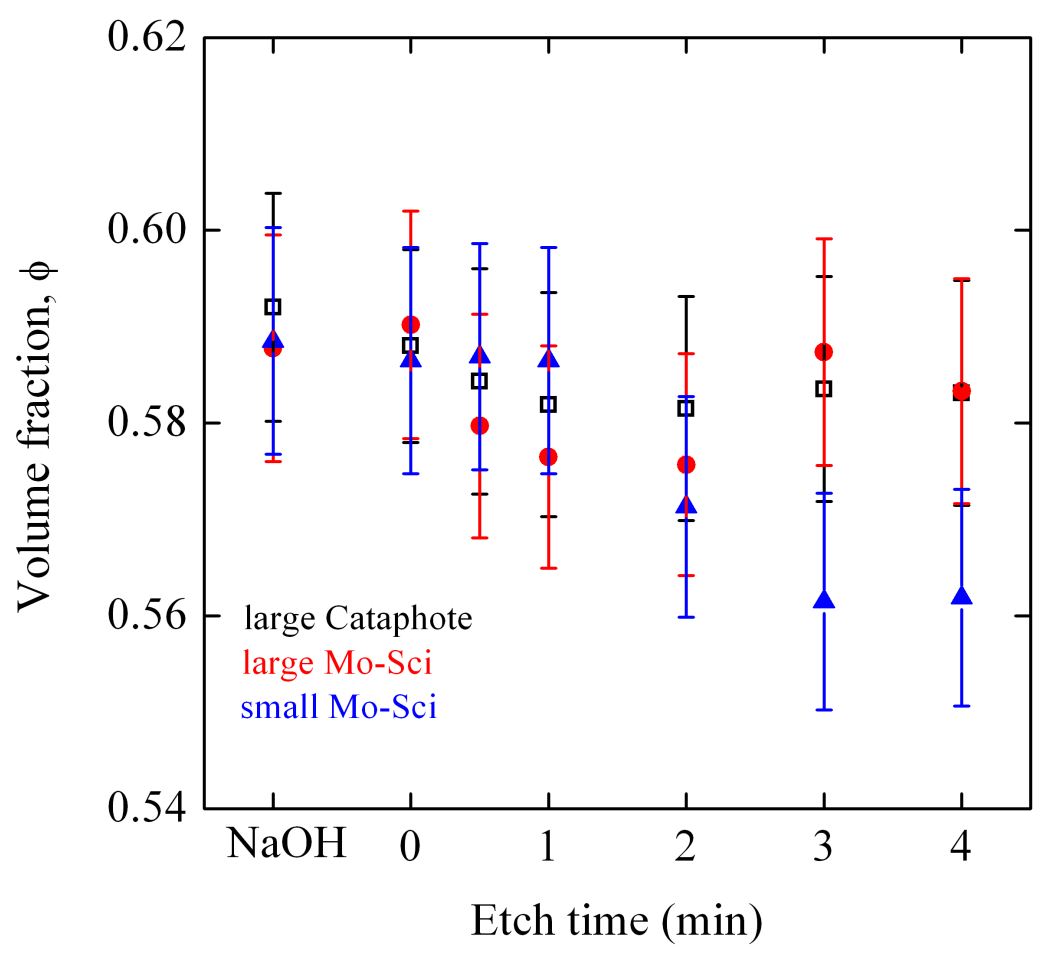

Figure 3.10.: $\quad \phi$ as a function of roughening etch time after subjection to the consolidation stress $\left(\sigma_{\text {pre }}=3150 \mathrm{~Pa} \pm 60 \mathrm{~Pa}\right)$.

scale (a millimetre scale glued to the cell); the slope gives the area of the cell. An underwater packing of grains is (re)produced on an LDS V409 shaker. The height of the packing is measured with a precision of $\pm \frac{1}{19} \mathrm{~mm}$ using a camera. In this way, $\phi$ is determined to within an error of $\pm 9 \times 10^{-3}$.

Next, $\alpha_{m s}$ is measured. For this, the container is transferred to a board which is hinged at one end and raised slowly at the other, under computer control, by a stepper motor (figure 3.14). A black-and-white CCD with 1 Megapixel and 8-bit depth is used to record the surface of the granular sample while the angle of the container is increased. The camera and an LED light source are mounted such that they do not move relative to the container, thus avoiding spurious results from shadows. The camera is triggered as the stepper motor starts and it takes one photograph every $3 \mathrm{~s}$, corresponding to an angular resolution of $0.5^{\circ}$. A calibration in which a stopwatch was photographed showed that jitter was irrelevant at this frame rate.

Image processing is then used to determine between which two frames an avalanche has occurred. Subsequent images of the surface of the sample are 


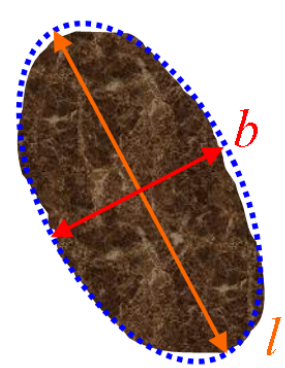

Figure 3.11.: Sketch to demonstrate measuring asphericity using the Camsizer: an elliptical fit (blue dotted line) is made to the image of the grain; $l$ and $b$ are the axial lengths of the ellipse.

subtracted. Figure 3.15 shows the absolute change in grey value (in bits) as a function of frame number. In this particular run, this information is already enough to detect the avalanche, but further image processing makes the method robust against single rolling grains and spatial inconsistencies in illumination. To this end, the subtracted images are binarised; the threshold for binarisation is taken as the mean grey value for a subtracted image of the stationary packing plus four standard deviations. This is shown in figure 3.16. The binarisation threshold is calculated anew for each experimental run, in order to allow for possible changes in illumination or exposure time.

Next, connected moving pixels are detected. We define an avalanche as a continuous moving area of more than one grain (more than 50 pixels with our optics and set-up). The subtracted image of an avalanche and its counterpart after binarisation and object identification are shown in figure 3.17. From the frame number at which the avalanche occurs, we know $\alpha_{m s}$.

\subsubsection{Results}

The angle of maximum stability as a function of $\phi$ is shown in figure 3.18 . We see that the smoother grains pack consistently denser than the rough grains. In both cases, a clear dependency of $\alpha_{m s}$ on the volume fraction can be observed. The effect is strongest for the roughest grains: here, the angle increases from $29^{\circ}$ to $41^{\circ}$ in the range $0.55<\phi<0.58$.

\subsection{Conclusion}

In this chapter, we measured the effect that grain roughness has on inter-particle friction.

The underwater angle of repose, $\alpha_{r}$, and thence the coefficient of inter-particle friction, $\mu$, was measured (section 3.1). The small Mo-Sci grains showed a mono- 


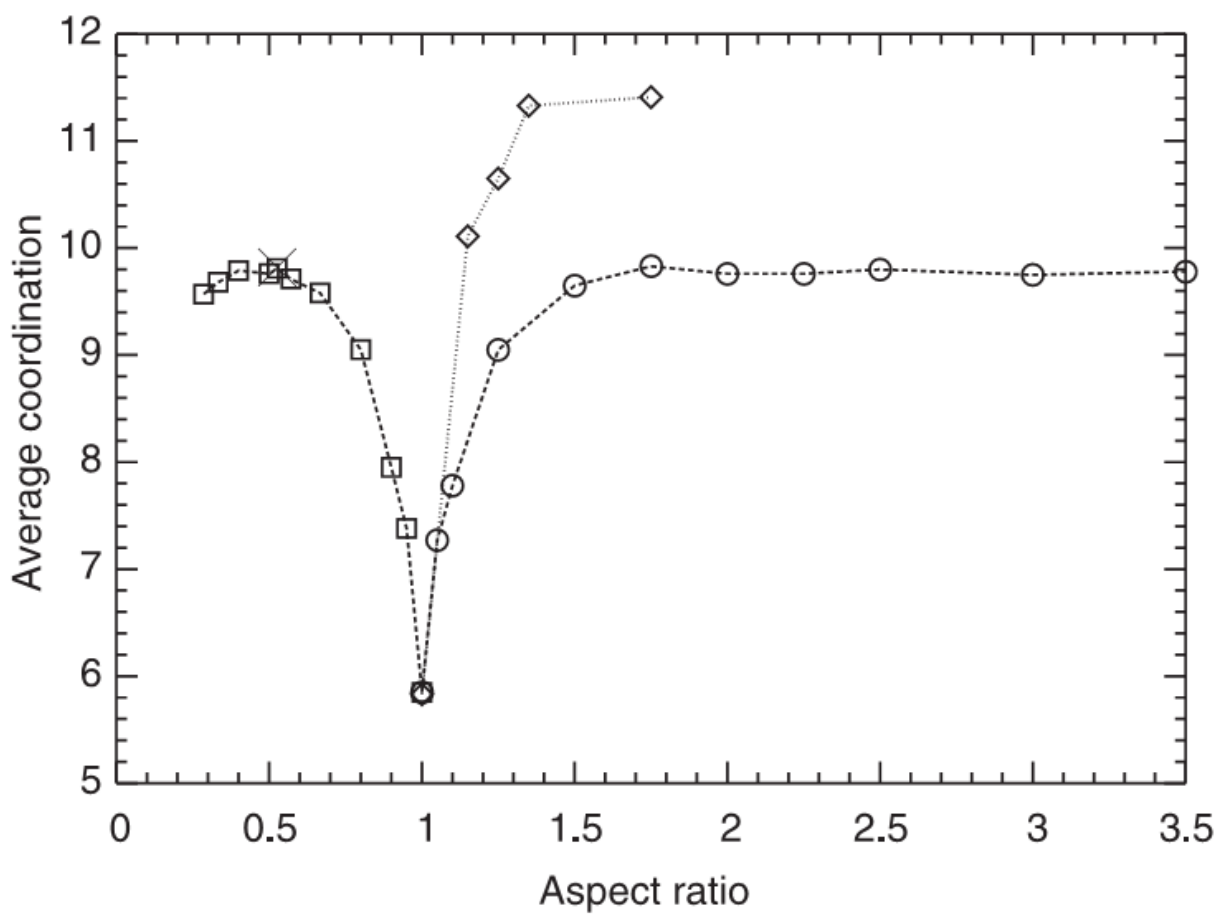

Figure 3.12.: The mean contact ("average coordination") number, $z$, as a function of particle aspect ratio, $a . z$ is the average number of inter-particle contacts per grain. The contact number of simulated 3D packings of prolate (circles) and oblate (squares) spheroidal particles without friction are shown. The diamonds are for frictionless ellipsoids and the cross is an experimental result for a packing of $m \mathscr{E} m$ sweets. Image taken from [61].

tonic increase in $\alpha_{r}$ with etch time with a rate of about $0.5^{\circ} /$ minute. The two larger types of grain - Cataphote and large Mo-Sci- had a constant $\alpha_{r}$ for all roughening etch times $\geq 30 \mathrm{~s}$. This behaviour looked similar to the vertical roughness length scale, $\xi_{\text {vert }}$ as a function of etch time (section 2.3). This led us to speculate that $\xi_{\text {vert }}$ could be the relevant length scale in dictating the frictional properties of grains. In section 3.1.2, the relaxation of the slope of the pile was shown to depend on particle roughness. Of the two types of grains tested, the smoothest grains came to a stop much later than the rougher grains, with a relaxation time three times greater. We suggested that this method of measuring friction could be used in situations in which $\alpha_{r}$ and $\alpha_{m s}$ are inaccessible.

The measurements of $\alpha_{r}$ were compared to the angle of internal friction in stationary flow, $\alpha_{s f}$, in section 3.2. $\alpha_{s f}$ was measured using a ring shear tester and the results gave a qualitative verification of the angle of repose measurements.

In sections 2.3 and 3.2 , we observed that there was a discrepancy between the friction angles, $\alpha_{r}$ and $\alpha_{s f}$, and the roughness length scale, $\xi_{\text {vert }}$, for the large Mo-Sci and Cataphote grains. In section 3.2.1, we suggested that this could be 


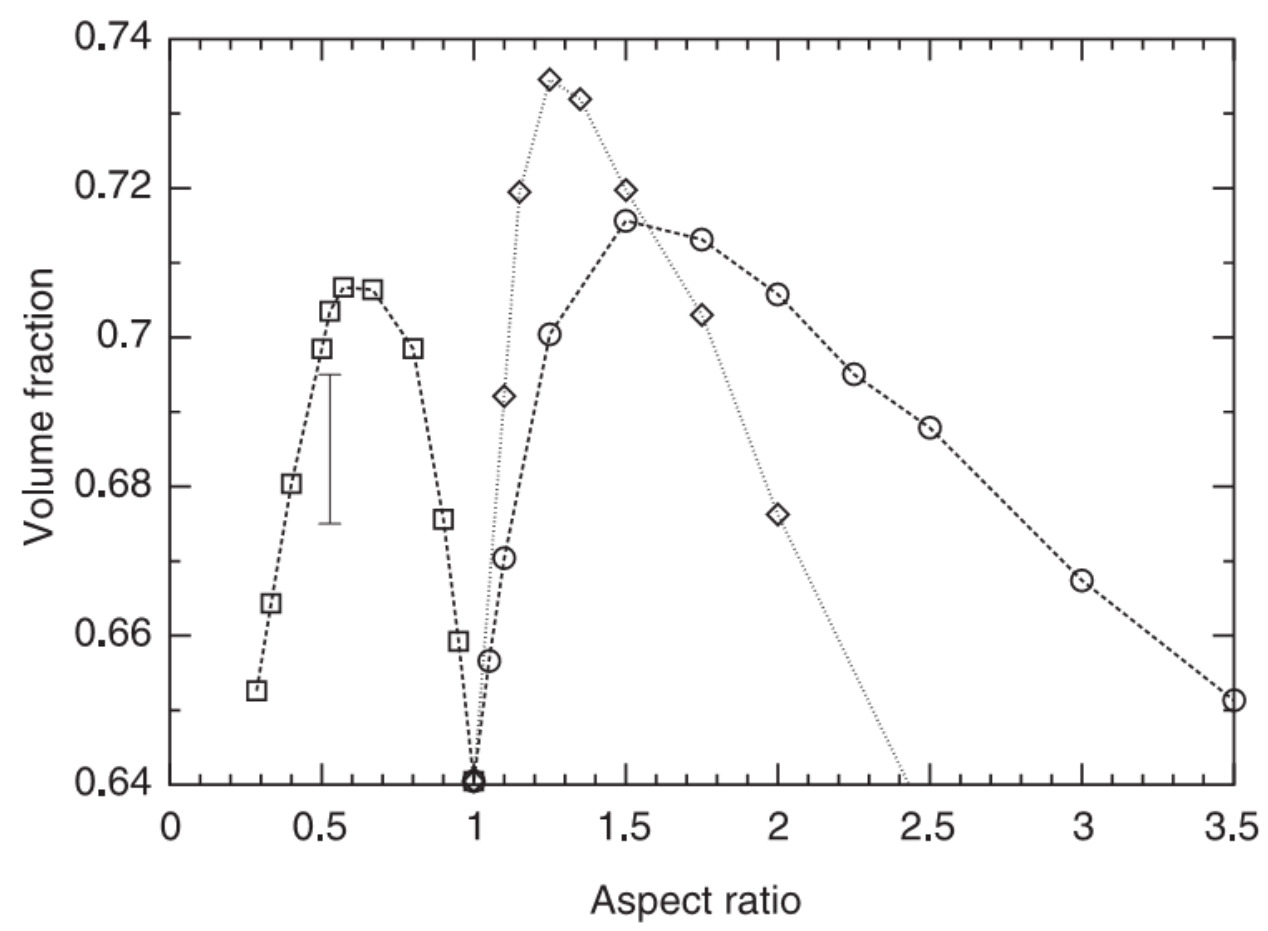

Figure 3.13.: The effect of asphericity on volume fraction. Shown are the densities of simulated 3D packings of prolate (circles) and oblate (squares) spheroidal particles without friction, as a function of the aspect ratio $a=l / b$. The diamonds are for frictionless ellipsoids and the error bar is an experimental result for a packing of $m \varangle m$ sweets. Image taken from [61].

because of different fractions of aspherical grains in the two grain types. The asphericity was measured using a Camsizer and it was seen that the average aspect ratio, $\langle a\rangle$, was greater for the Cataphote than for the Mo-Sci grains. We suggested two mechanisms by which increasing asphericity could bring about the discrepancy: by increasing the contact number, $z$, or by increasing the volume fraction, $\phi$.

The influence of grain roughness on $\phi$ was investigated in section 3.3 . It is to be expected that grains with greater inter-particle friction produce less dense packings than smoother grains. The measurements affirmed this with $\phi$ being consistently smaller for rough grains. We investigated the effect of $\phi$ on granular pile stability by measuring the angle of maximum stability for various volume fractions for two batches of grains. It was shown that $\alpha_{m s}$ increases monotonically with $\phi . \phi$ was seen to effect $\alpha_{m s}$ most strongly for the roughest grains.

In this and the previous chapter, we have shown that chemical etching of grains can be used to tune their roughness and thence their frictional properties. These results were published in September 2011 in reference [62]. 


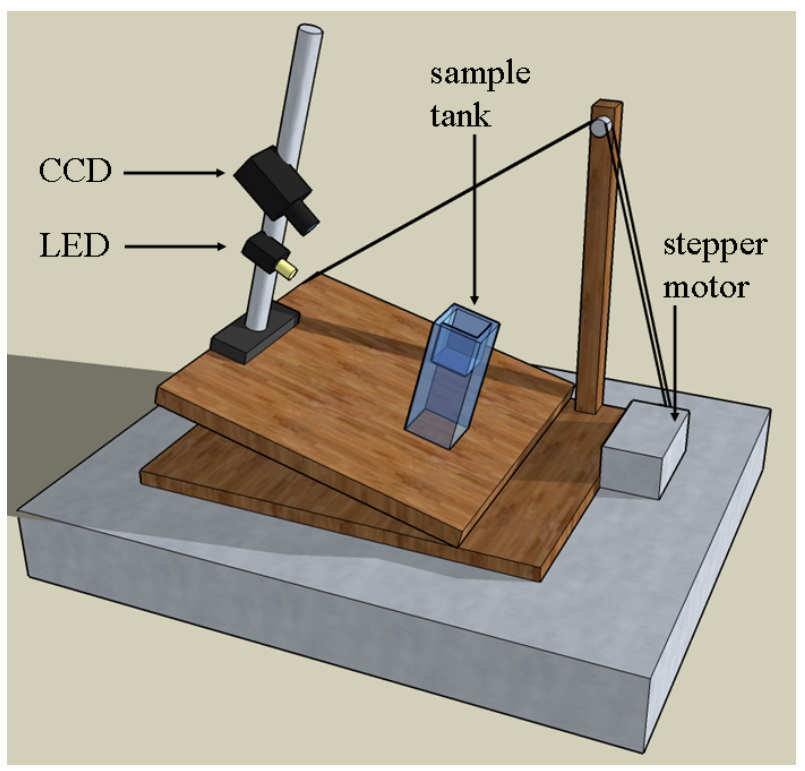

Figure 3.14.: The apparatus for measuring the angle of maximum stability. A granular sample in a water tank is prepared by shaking and mounted on the board. The board is then raised at one edge by a stepper motor. The camera monitors the onset of avalanching.

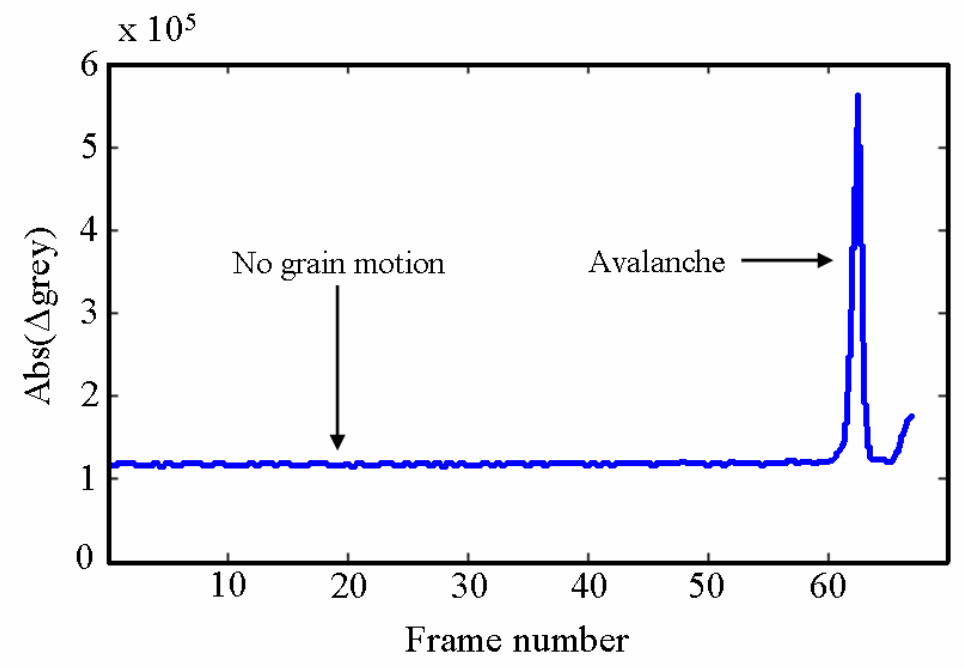

Figure 3.15.: The absolute change in grey value (in bits) as a function of frame number. The tail after the avalanche is due to the shaking of the bed caused by the rapid lowering of the board. 


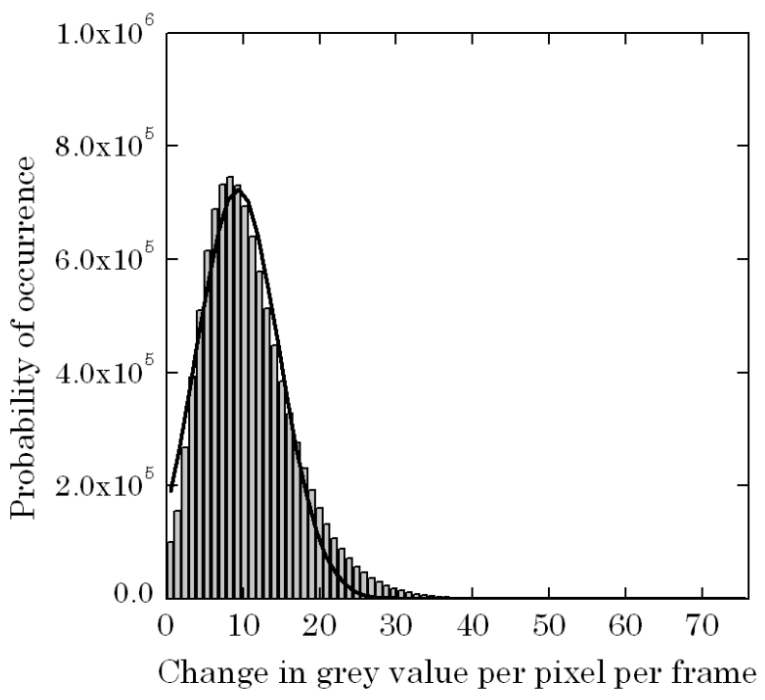

Figure 3.16.: The grey level histogram of the subtracted image of the packing before it avalanches. Since there is no motion between the two frames, the grey values we see here are all due to noise in the illumination. The threshold for binarisation is taken as the mean grey value plus four standard deviations. The line is a Gauss fit, but this is not used in determining the threshold.

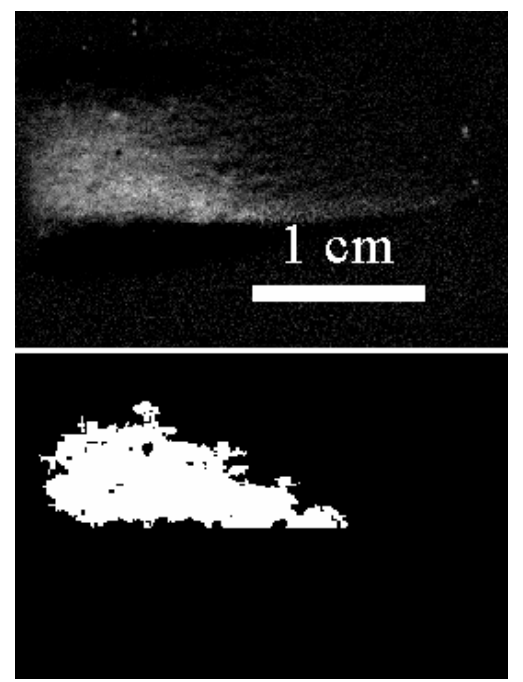

Figure 3.17.: Top: subtracted image of an avalanche. The brighter the pixel, the greater the difference between the two frames. Bottom: connected moving pixels in the same avalanche avalanche (white). The direction of avalanche motion in the images is downwards; the sharp edge shows where it has reached the side of the cell. 


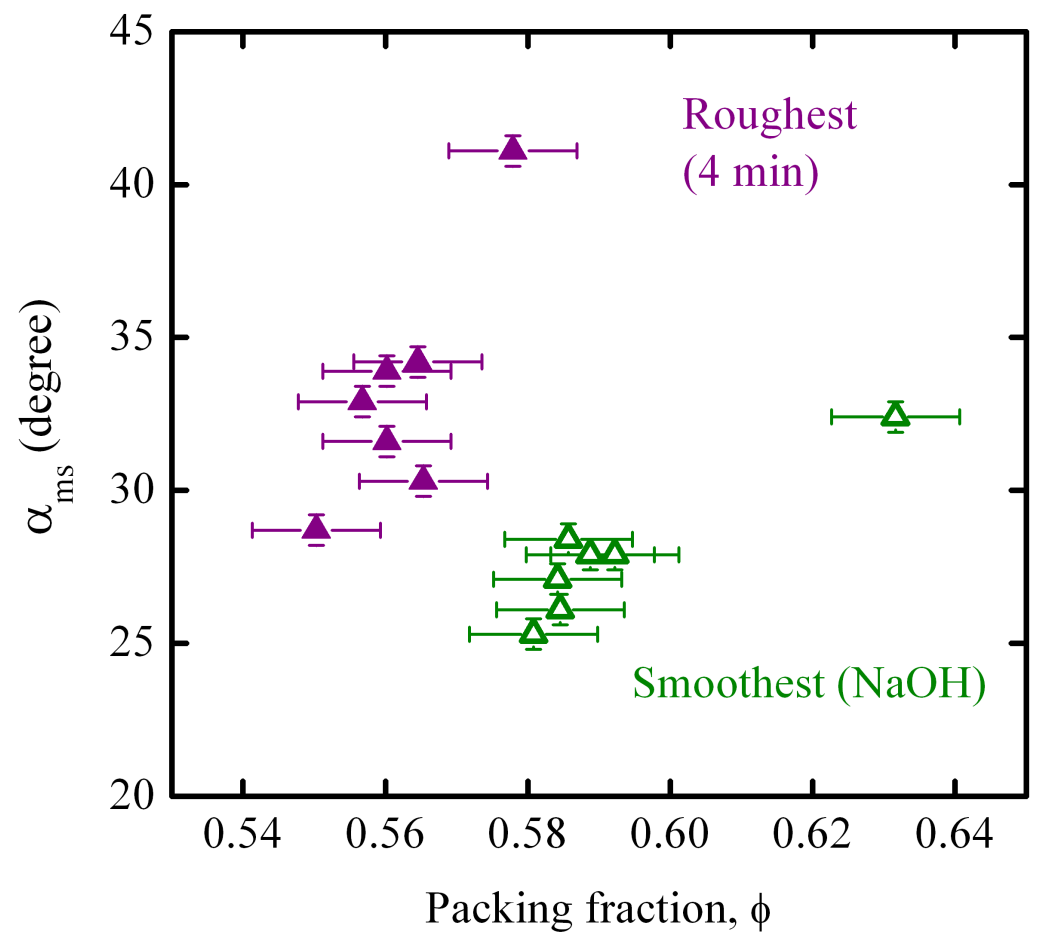

Figure 3.18.: $\quad \alpha_{m s}$ as a function of $\phi$ for the smoothest $(\mathrm{NaOH})$ and roughest (4 minutes in $\mathrm{NH}_{4} \mathrm{FHF}$ ) small Mo-Sci grains. 



\section{Part II.}

\section{Measuring granular dynamics with light transport}





\section{Foreword to part 2}

Part II of this thesis looked at changing the frictional properties of a granular material with the aim of making friction a control parameter in the study of granular statics and dynamics. Some aspects of granular statics we have already looked at (packing densities: section 3.3; surface nucleation: section 2.5), others are looked at in section 6.4 (force networks) and section 6.2 (wetting of granular media). This part of the thesis looks at some of the dynamics of granular media.

A mechanics approach to granular dynamics looks at velocities, momenta, torques, accelerations and forces. Our approach is not to look at these microscopic mechanics, but to take a statistical mechanics approach and look at a macroscopic state variable: the kinetic granular temperature. This approach was first pursued in a rigorous mathematical framework by Jenkins and Richman in 1985 [18, and is an extension of equilibrium thermodynamics and the kinetic theory of Boltzmann [63].

The physics of a granular "fluid" is significantly different to an ideal gas in two ways: the first is that the particles have a finite size, which means that rotational degrees of freedom are also present (as is the case for a molecular gas). However, unlike the molecular gas, we may not assume equipartition of energy between the degrees of freedom [64]. The second is that collisions occur between grains. These collisions are inelastic, which leads to correlations which manifest themselves in the microscopic mechanics of the system (velocities, momenta, etc.). The inelastic nature of the particle collisions also means that a granular system needs the constant injection of energy in order to remain in the fluid state; in the language of thermodynamics, it is an open system. If energy is put into the system at the same rate as it is dissipated, we speak of a non-equilibrium steady state. There are many ways of driving a granular system, and these are outlined below. In this thesis, we study the physics of the steady state by looking at kinetic granular temperature $4^{4}$ The concept of kinetic granular temperature is outlined below.

\section{Driving granular media}

There are many methods to choose from when deciding how to inject energy into a granular experiment.5 In chapter 1, our scientist provided potential energy to

\footnotetext{
${ }^{4}$ Two other definitions of granular temperature are discussed in section 6.3

${ }^{5}$ Especially when the experiment is a computer simulation.
} 
her sand by inverting the hour glass. This is the type of energy injection we use in chapter 3 when measuring the angles of repose and maximum stability. A system undergoing this type of driving is far from being in a steady state, as we saw, for example, in the rotating drum experiment of Courrech du Pont et al. 3.1 .

It is also common to drive a granular experiment by injecting kinetic energy. This might be done by shearing, shaking or fluidising. We use shearing in section 3.2. where we measure the angle of internal friction. Shaking is used for experiments at high granular temperatures, since one needs to provide an acceleration $\Gamma \geq g$, large enough to overcome the acceleration due to gravity, $g$. This method has the disadvantage that it adds energy at the boundary of the sample and relies on inter-particle collisions to transmit the energy to the centre. Since granular collisions are inelastic, this leads to inhomogeneous driving [19]. Pumping a fluid through a sample is a more homogeneous way of injecting energy and it has the additional advantage of allowing access to the low-temperature experimental regime, where glassy dynamics such as caging, creep and dynamical heterogeneities are expected to occur [65]. However, it has the disadvantage that there are hydrodynamic interactions between the grains and the fluid. For our experiments on granular temperature, we use a water-fluidised bed. This set-up is described in section 4.1

\section{Kinetic granular temperature}

The kinetic granular temperature, $T_{\text {kin }}$, is taken from kinetic theory. The kinetic energy of an ideal gas is given by

$$
E_{\text {kin }}=\frac{3}{2} k_{B} T=\left\langle\frac{1}{2} m \vec{v}^{2}\right\rangle,
$$

where $E_{\text {kin }}$ is the kinetic energy, $k_{B}$ Boltzmann's constant, $T$ temperature, $m$ particle mass and $\vec{v}$ the velocity. $\langle\cdots\rangle$ indicates ensemble averaging.

There are three important considerations that we need to make when applying kinetic theory to granular materials. These are:

First, in a granular gas, $k_{B}$ loses its meaning, so we simply remove it from our consideration, making the unit of $T_{\text {kin }}$ Joule [19].

Second, in a granular medium grains cannot be treated as points traversing three-dimensional space: they have a finite size, which means that, as well as their three translational degrees of freedom, they have two degrees of rotational freedom [34].

Third, we cannot assume equipartition of energy between the degrees of freedom [64].

This leads us to the definition 


$$
E_{\text {kin }}=\frac{6}{2} T_{\text {kin }}=E_{t r}+E_{\text {rot }}=\left\langle\frac{1}{2} m \vec{v}^{2}\right\rangle+\left\langle\frac{1}{2} I \vec{\omega}^{2}\right\rangle,
$$

where $E_{t r}$ and $E_{\text {rot }}$ are the kinetic energies associated with the three independent translational and the two independent rotational degrees of freedom. $I$ is the moment of inertia and $\vec{\omega}$ the angular velocity of the grain [34].

In this section of the thesis, we describe our approach to measure $T_{\text {kin }}$. In chapter 4. we report a method of driving a granular system using water fluidisation, and present our method of measuring $T_{\text {kin }}$ using diffusive light transport. In the context of this thesis, diffusive light transport is used as a means of measuring granular dynamics, but it is a very interesting topic in its own right. For this reason, we discuss it rather expansively in chapter 5 .

A note about the grains we use in this part: soda-lime glass grains from Whitehouse Scientific were used at first, but they had a large proportion of aspherical particles. For this reason, later measurements were made using the same unetched Cataphote and Mo-Sci grains that were used in part I of the thesis. Additionally, barium titanate (BaTi) glass spheres from $\mathrm{Mo}-\mathrm{Sci}$ were used, in order that the effect of refractive index could be investigated. 



\section{Kinetic granular temperature in a driven suspension}

In the first part of this chapter, we outline the function of the water-fluidised bed as a means of injecting energy into a granular system. In the second part, we describe how the kinetic granular temperature, $T_{\text {kin }}$, can be measured using laser speckle, and present our measurements of the time scale of granular motion, $\tau$. Together with a length scale, $l^{*}, \tau$ is related to the granular temperature as [66]

$$
{ }_{\frac{6}{2}} T_{\text {kin }}=\left\langle\frac{1}{2} m \vec{v}^{2}\right\rangle+\left\langle\frac{1}{2} I \vec{\omega}^{2}\right\rangle \propto\left(\frac{l^{*}}{\tau}\right)^{2} .
$$

$l^{*}$ is the transport mean free path. This quantity is discussed at length in chapter 5 .

\subsection{Fluidised bed}

We use a water-fluidised bed to drive our granular media in which we measure measure $\tau$ and $l^{*}$. Fluidised granular beds are by no means a new technology. They have been used since at least the 1920s for purposes as diverse as sewage treatment and the catalytic cracking of hydrocarbons [67]. The principle of function of a water-fluidised bed (sketched in figure 4.1) is a simple one: water is pumped with flow rate $Q$ from below through a distributor into the granular packing, which expands until it reaches a constant volume fraction, $\phi$. The grains are agitated continually and homogeneously with an energy $T_{\text {kin }}$.

Consider a fluidised granular bed of cross-sectional area $A$ that is undergoing constant driving with flow rate $Q$ and has attained a constant $\phi$. The gravitational force acting on a spherical grain is balanced by the buoyant and drag forces such that 68, 69]

$$
F_{\text {drag }}=F_{\mathrm{g}}-F_{\text {buoy }} .
$$

The gravitational force is given by

$$
F_{\mathrm{g}}=\rho_{g} g \frac{\pi}{6} d^{3}
$$

where $\rho_{g}$ is the material density of the grain, $d$ is its diameter and $g$ is the acceleration due to gravity. The buoyancy force is given by 


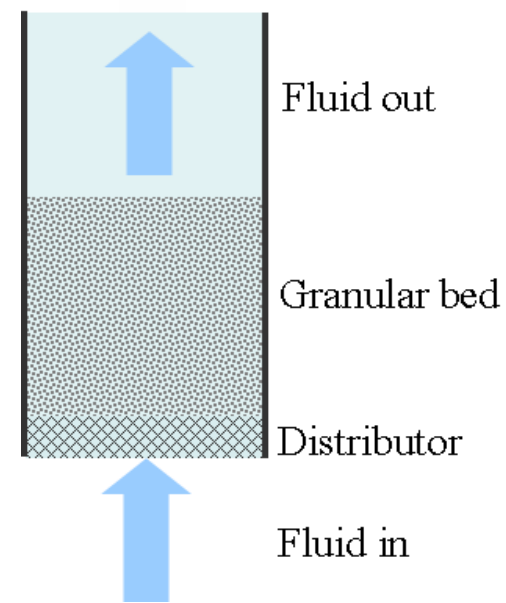

Figure 4.1.: Principle of function of a fluidised bed: the fluid flows up through a distributor into the granular bed.

$$
F_{\text {buoy }}=\left(\phi \rho_{g}+(1-\phi) \rho_{w}\right) g \frac{\pi}{6} d^{3} .
$$

Here, $\left(\phi \rho_{g}+(1-\phi) \rho_{w}\right)$ is the density of the granular suspension; the subscripts $g$ and $w$ refer to grain and water. And the force due to Stokes drag on the grain is given by

$$
F_{\text {drag }}=3 \pi d \mu_{w} U f(\phi),
$$

where $\mu_{w}$ is the viscosity of the water, $U=Q / A$ is the superficial fluid velocity, and $f(\phi)$ is a function of unknown form ${ }^{1}$ that takes account of the presence of the surrounding particles. Substituting equations 4.3, 4.4 and 4.5 into equation 4.2 gives us the expression 68

$$
3 \pi d \mu_{w} U f(\phi)=g \frac{\pi}{6} d^{3}\left(\rho_{g}-\rho_{w}\right)(1-\phi) .
$$

This can be used to calculate the flow rate needed to obtain a given volume fraction:

$$
Q(\phi)=A v_{\text {terminal }} \frac{1-\phi}{f(\phi)} .
$$

$v_{\text {terminal }}$ is the terminal velocity attained by a single sphere sinking through the water:

\footnotetext{
${ }^{1}$ There are engineering approximations for the function. For example, Carman gives the relation $f(\phi)=10 \frac{\phi}{(1-\phi)^{2}}[69$.
} 


$$
v_{\text {terminal }}=\frac{g d^{2}\left(\rho_{g}-\rho_{w}\right)}{18 \mu_{w}}
$$

where $\mu_{w}$ is the viscosity of water. Therefore by careful tuning of the experimental control parameters $Q$ and $\rho_{w}$, it is possible to change either $Q$ (and thence $T_{\text {kin }}$ ) and keep $\phi$ constant or change $\rho$ (and thence $\phi$ ) and change $T_{\text {kin }}$.

In this work, we change only $Q$, so it must be borne in mind that we are simultaneously changing two variables, $T_{\text {kin }}$ and $\phi$.

Because there is no real consensus on what form $f(\phi)$ takes [69, 70], we measure the dependence of $\phi$ on $Q$ experimentally. For this and all subsequent experiments with fluidised beds, we use water with a temperature of $21^{\circ} \pm 1^{\circ}$, keeping $\rho$ at a constant $998 \mathrm{~g} / \mathrm{cm}^{3} \pm 0.2 \mathrm{~g} / \mathrm{cm}^{3}$ and $\mu_{w}=1.02 \mathrm{mPas} \pm 0.02 \mathrm{mPas}$. The pump is a Materflex Micropump gear pump with 180 - 3600 r.p.m. Our distributor is made of two plates of sintered $\sim 40 \mu \mathrm{m}$ bronze beads $5 \mathrm{~mm}$ thick. $\phi$ was measured in the same way as for the $\alpha_{m s}$ measurements in section 3.3 .

The influence of $Q$ on $\phi$ measured for soda-lime glass spheres $250 \mu \mathrm{m}-300 \mu \mathrm{m}$ in diameter is shown in figure 4.2. A monotonic relationship is clearly observed. This shows that the water-fluidised bed is a successful technique, allowing us to control $\phi$ with great precision.

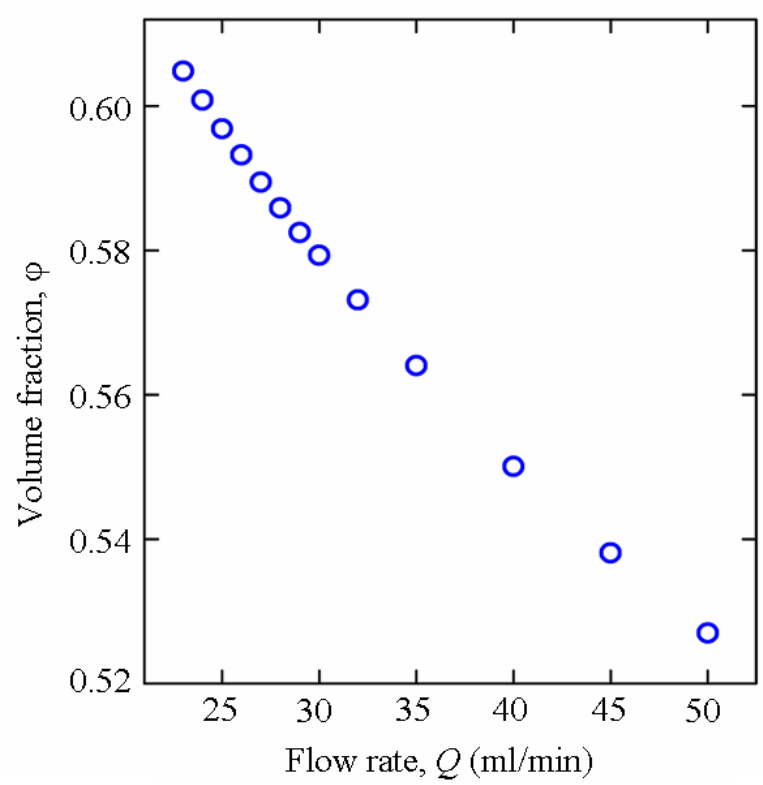

Figure 4.2.: Volume fraction of a water-fluidised granular suspension as a function of flow rate. The grains used were soda-lime glass spheres $250 \mu \mathrm{m}-300 \mu \mathrm{m}$ in diameter made by the company Whitehouse Scientific. The error on $\phi$ is \pm 0.01 . 


\subsection{Methods to measure $T_{\text {kin }}$}

To measure the kinetic granular temperature, one needs to measure the average velocity of the grains (equation 4.1). The most direct way of doing this would be to measure the individual three-dimensional trajectories of the grains in good temporal resolution. But due to crowding, overlapping and velocity constraints, this is hardly a trivial enterprise. In this thesis, we use laser speckle to measure kinetic granular temperature; the method is described in section 4.3 . But first, we will briefly review three other experimental methods.

Nuclear magnetic resonance (NMR) has been used since the 1990s to look at granular dynamics. Nakagawa et al. used NMR to look at spatial concentration and velocity profiles of the three-dimensional flow of mustard seeds in a rotating drum with a view to understanding segregation and pattern formation [71]. Lasič et al. used a version of $\mathrm{NMR}^{2}$ to look into an air-fluidised bed of mustard seeds and obtained non-Gaussian velocity distributions [72]. However, it is possible that their measurements are strongly influenced by granular convection rolls, making their results difficult to interpret. Both of these NMR techniques have the advantage that they could, in principle, allow the simultaneous measurement of $\vec{v}$ and $\vec{\omega}$.

Wildman et al. used positron emission particle tracking to follow the trajectory of a single grain in a vibrated granular bed [73]. Although the authors used very long traces of a single grain to measure granular temperature, the success of the method hinges on the assumption of ergodicity; to make this assumption for a granular system is not without its risks.

Visual tracking methods are limited to 2D or quasi 2D systems. Losert et al. investigated a vertically shaken granular monolayer and used high-speed camera tracking to follow the $2 \mathrm{D}$ projections of the $3 \mathrm{D}$ grain trajectories [74]. They observed that $T_{\text {kin }}$ changes as a function of area fraction and that the velocity distribution is non-Gaussian. Nichol and Daniels investigated a two-dimensional granular system of bidisperse discs levitating on an air-hockey table [75]. In a 2D experiment such as this, the trajectories and rotations of all discs can be followed for the entire duration of the experiment, which enabled the authors to measure translational and rotational velocity distributions. A key result of this work was the observation of equipartition of energy between translational and rotational degrees of freedom.

\footnotetext{
${ }^{2}$ high-speed modulated gradient spin echo tomography
} 


\subsection{Measuring $T_{\text {kin }}$ using diffusing wave spectroscopy}

Since we are interested in average speeds, we can employ a method to measure $T_{\text {kin }}$ that gives us an average scalar displacement as a function of time. This sidesteps the problems encountered in particle tracking experiments. The method to do this carries various names, which describe various flavours of the method, but we will refer to it here as diffusing wave spectroscopy, or DWS for short. The resolution of the method is typically of the order of $10 \mathrm{~nm}$ and $50 \mu \mathrm{s}\left[76\right.$ ] $?^{3}$

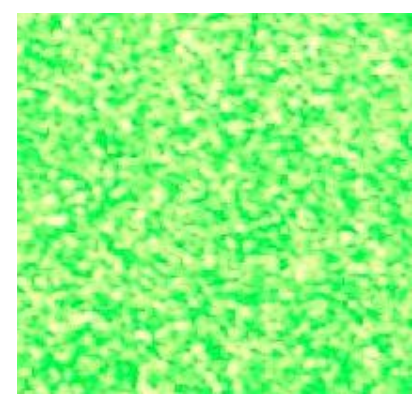

Figure 4.3.: Colour photograph of a laser speckle pattern $(\mathrm{ca} .1 \mathrm{~cm} \times 1 \mathrm{~cm})$ formed through frosted glass.

When a transparent medium is dispersed in another transparent medium of different refractive index, light passing through the dispersion will be scattered. We make this observation every day when we look at milk (fat droplets dispersed in water), clouds (water droplets in air) or shaving foam (air bubbles in water). Although fat, water and air are transparent, we cannot see through the dispersion. The same thing happens in a pile of sand: although the grains are made of transparent silica (natural sand) or glass (the grains used in this thesis), we cannot see through the pile. Instead, light is scattered and the medium appears to be white.

If we shine coherent light into such a scattering medium and look at the backscattered or the transmitted light, we will observe a complex interference pattern called speckle. An example of a speckle pattern is shown in figure 4.3 . We now shine a laser through such a medium in motion 4 The time needed by the light to pass through the medium is much shorter than $\tau$, the time it takes for the grains to move enough to change the speckle pattern [77]. For the speckle

\footnotetext{
${ }^{3}$ The resolution of the method is limited by the wavelength of the light and the speed of the camera used.

${ }^{4}$ Milk and clouds are both made of droplets so tiny that they undergo Brownian motion, so they are always in motion. Additionally, clouds are very turbulent places. The shaving foam may also be in motion at much longer time scales due to bubble rearrangements, coalescence and bursting.
} 
pattern to be visible, the exposure time of our camera, $T$, must also be shorter than $\tau$. If $T>\tau$, the pattern blurs so that each camera pixel registers the same intensity, $I=\langle I\rangle$. The $\langle\cdots\rangle$ indicates an spatial average over the whole field of view.

Thus the visibility of a speckle pattern at a given $T$ encodes information about $\tau$. To define visibility quantitatively, one looks at $V(T)$, the normalised variance of the speckle pattern as a function of exposure time. Appendix C shows how $\tau$ is obtained from $V(T)$.

Figure 4.4 shows experimental measurements of $\tau$ as a function of volume fraction obtained by measuring $V(T)$ for various $Q$. The grains are unetched large Mo-Sci spheres. The ordinate of the graph, $(1 / \tau)^{2}$, is proportional to $T_{\text {kin }} / l^{* 2}$ (equation 4.1).

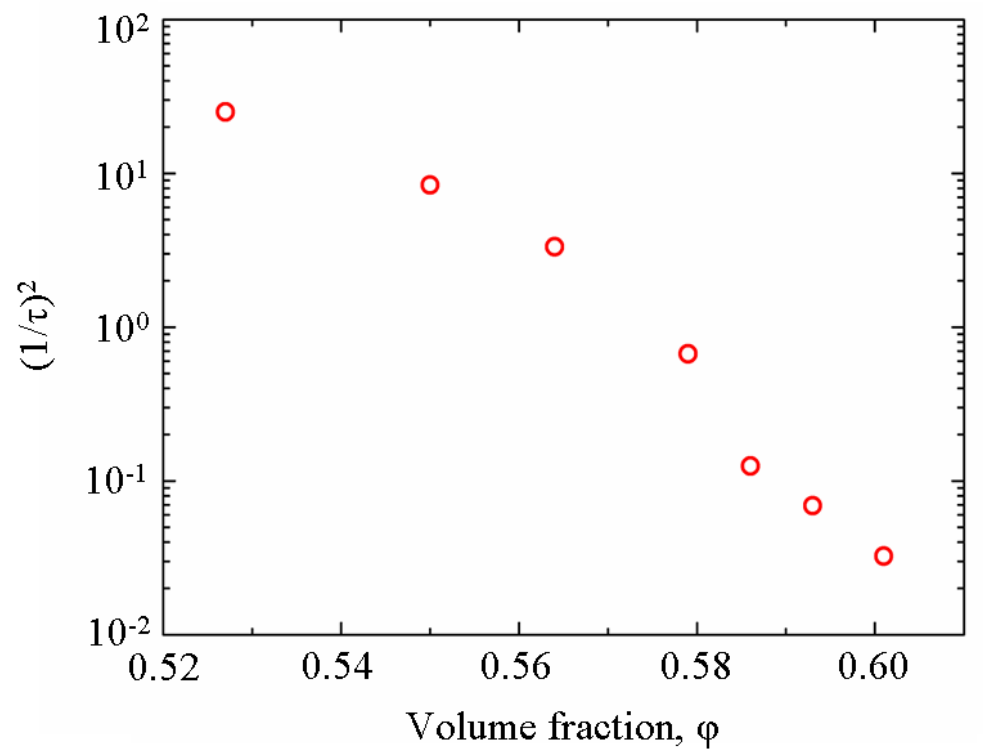

Figure 4.4.: $\quad(1 / \tau)^{2}$ measured for large Mo-Sci grains at flow rates $24 \mathrm{ml} / \mathrm{s} \leq Q \leq 50$ $\mathrm{ml} / \mathrm{s}$. The unit of $\tau$ is second. Illumination was a Nd:YAG laser with $P=3 \mathrm{~W}$ and $\lambda=532 \mathrm{~nm}$. The camera was a Phantom camera with a frame rate of $10,000 \mathrm{~Hz}$ and an exposure time of $90 \mu \mathrm{s}$. Measured by Matthias Schröter.

In order to calculate $T_{\text {kin }}$ from $\tau$ data such in figure 4.4, one also needs a length scale. This is the transport mean free path, $l^{*}$, the length scale over which the light propagation direction is randomised. If we assume $l^{*}$ to be constant for all $\phi$, the ordinate of figure 4.4 is proportional to $T_{\text {kin }}$. However, one would expect $l^{*}$ to depend on $\phi 5^{5}$ For this reason, it is essential to measure $l^{*}$.

It turns out that this quantity is rather tricky to measure. The next chapter is dedicated to the description and measurement of $l^{*}$.

${ }^{5}$ This is discussed in section 5.3 . 


\section{A note of caution}

The value for $\tau$ measured by DWS is an average time scale of granular movement that does not discriminate between degrees of freedom. Yet there is no reason for us to suppose that the time scales for translation and rotation are the same, nor may we assume equipartition between the degrees of freedom [33]; Brilliantov et al. showed that the ratio of rotational to translational temperature, $r \equiv T_{\text {rot }} / T_{t r}$ depends on grain roughness [34]. Indeed, we cannot even be certain that this quantity is the same for all $\phi$ [74] or driving strengths.

This weakness is exacerbated by the different sensitivity of DWS to the rotational and the translational degrees of freedom of the grains. The rotations of optically smooth, perfectly spherical grains would be invisible to DWS and the $T_{\text {kin }}$ we would measure for such grains would describe only their translational kinetic energy. At the opposite extreme, the rotations of very rough or aspherical grains would have an influence on the speckle pattern that is at least equal to the contribution of their translational movements. Thus for experiments on real grains with finite roughness, the experiment's perception of $r$ is inherently skewed and we have no way of knowing to what extent. 



\section{Transport mean free path of light}

In chapter 4 we saw that, in order to measure the kinetic granular temperature, one needs to measure a length scale we called $l^{*}$. Put simply, the transport mean free path, $l^{*}$, is the random walk step length of a photon ${ }^{1}$ diffusing through a scattering medium [78]. To understand this picture, we need first to explain diffusive light transport.

Let us start by looking at what happens when light encounters a single scattering particle. For small scatterers $(R \ll \lambda$, where $R$ is the radius of the scatterer and $\lambda$ the wavelength of light), light is scattered elastically; this is called Rayleigh scattering and it is described by wave optics. In this case, the incoming light has a high probability of being scattered through a large angle, which means that a single Rayleigh particle is enough to significantly change the course of the light. If we translate this to the random walk picture, $l^{*}$ is simply the mean distance between scattering events, $l_{s}$. For large scatterers $(R \gg \lambda)$, we are in the limit of geometrical optics and the light is strongly forward scattered: it has only a tiny probability of being scattered through a large angle. This is called Mie scattering. Here, several scattering events are necessary to make a ray significantly change its course, making $l^{*}>l_{s} \stackrel{2}{2}^{2}$ In our experiments, where $R \sim 100 \mu \mathrm{m}$ and $\lambda=532$ $\mathrm{nm}$, we are in the Mie regime [79].

Now let us look at how light propagates through a dense, random distribution of scatterers. Light scattering can be dealt with analytically if we assume that light transport in a scattering medium is diffusive. $l^{*}$ is defined as a function of the scattering angle, $\theta$, and the scattering mean free path, $l_{s}$ by the expression 80 .

$$
l^{*}=\frac{l_{s}}{1-\langle\cos \theta\rangle} .
$$

In the isotropic scattering limit, $\langle\cos \theta\rangle$ tends to zero and $l^{*}$ to $l_{s}$. In the case of our glass grains, forward scattering (between the angles $\pi \leq \theta \leq \pi$ ) is more probable, so $0<\langle\cos \theta\rangle<1$ and $l^{*}>l_{s}$.

\footnotetext{
${ }^{1}$ When we speak of photon in this chapter, it is not with its quantum mechanical nature in mind, but rather because, when describing diffusive light transport, it is often helpful to think of light as diffusing, point-like particles.

${ }^{2}$ The scattering probabilities of some relevant scatterers are calculated in appendix E
} 
There are three experimental methods suitable for measuring $l^{*}$ in granular systems such as ours. In section 5.1, these methods are described; in section 5.2 , we present the experimental results we obtain from the three methods. In section 5.3. we compare our results to the most relevant literature results.

\subsection{Methods of measuring $l^{*}$}

\subsubsection{Measuring $l^{*}$ in transmission}

The diffusion approximation is the first important step in describing analytically the transmission of light through a scattering medium. When considering transmission through a sample, a second assumption is made. We assume that the light, rather than being coherent light coming from an external light source, is composed of incoherent photons injected into the sample locally at a given penetration depth, $z_{p} l^{*}$. We can then formulate the problem of light transmission as follows. Consider an infinite slab of a turbid medium, whose thickness, $L$, is much greater than $l^{*}$ (figure 5.1). The condition $l^{*} \ll L$ is necessary to ensure that we are working in the diffusive, multiply-scattering regime. There are four transport length scales that enter the theory [81]:

1. the penetration depth, $z_{p} l^{*}$, the distance into the slab at which we inject incoherent photons into the sample;

2. the extrapolation length, $z_{e} l^{*}$, defined as the distance outside the slab at which the photon concentration is assumed to extrapolate to zero. This is a consequence of the diffuse reflection at the boundaries (section 5.1.1);

3. the transport mean free path, $l^{*}$; and

4. the absorption length, $L_{a}=\sqrt{l^{*} l_{a} / 3}$, a diffusion-dependent length scale that accounts for absorption within the sample. $l_{a}$ is a material-dependent attenuation length scale [82].

We can describe the passage of light through the sample by the steady-state diffusion equation for the dimensionless energy density $n(\rho, z)$, where $z$ is the distance along the normal to the slab, measured from the origin, as illustrated in figure 5.1, and $\rho$ is the is the radial vector from the $z$-axis [83]:

$$
\nabla^{2} n(\rho, z)-\frac{n(\rho, z)}{L_{a}^{2}}=\frac{-3 J_{0}}{l^{*} c_{t}} \delta(\rho) \delta\left(z-z_{p} l^{*}\right) .
$$

Here, $c_{t}$ is the speed of light in the medium. The right hand side is the source term, expressed in terms of $J_{0}$, the total flux entering the sample. The source is a delta function at $\rho=0, z=z_{p} l^{*}$. The left hand side is the diffusion term minus a sink term characterised by the absorption length $L_{a}$. 


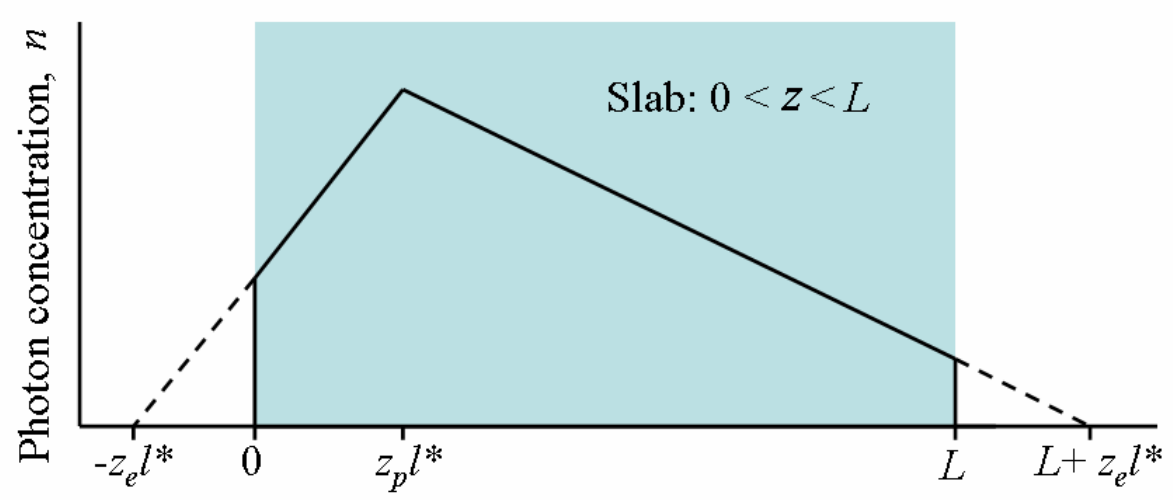

Distance into the sample, $z$

Figure 5.1.: The photon concentration, $n(z)$, sketched as a function of distance, $z$, for an infinite slab in which light transport is purely diffusive. The straight lines $n(z)$ are a solution of the diffusion equation 5.2 for weak absorption, $L_{a} \rightarrow 0$. Figure adapted from Lemieux, Vera and Durian [81].

Now for the boundary conditions. Since we assume the slab to be of infinite width and length, we impose $n(\rho=\infty, z)=0$. Since the only photon source in the problem is located at $z_{p} l^{*}$, any light entering the sample from the sides must be due to reflections from the boundaries, which arise from the mismatch of refractive indices there ${ }^{3}$ From the Fresnel reflectivities at the boundaries, we define an extrapolation length $z_{e} l^{*}$ to describe this, which is the length at which the photon concentration extrapolates to zero (figure 5.1). Thus our boundary conditions at the edges of the slab read [83]

$$
n(\rho, z)-\left.z_{e} l^{*} \frac{\partial n(\rho, z)}{\partial z}\right|_{z=0}=0
$$

at $z=0$, and

$$
n(\rho, z)+\left.z_{e} l^{*} \frac{\partial n(\rho, z)}{\partial z}\right|_{z=L}=0
$$

at $z=L$. The nature of $z_{e}$ is discussed in section 5.1.1.

We are interested in the total transmission of light through the sample. By solving equation 5.2 with the above boundary conditions and then integrating the transmitted flux over all $\rho$, Li et al. obtain the expression for the transmissivity 83 .

\footnotetext{
${ }^{3}$ In the absence of boundary reflections, $n(0)=n(L)=0$.
} 


$$
T(L)=\frac{\sinh \left(z_{p} l^{*} / L_{a}+\epsilon\right) \sinh (\epsilon)}{\epsilon \sinh \left(L / L_{a}+2 \epsilon\right)}
$$

where

$$
\epsilon=\frac{1}{2} \ln \left[\frac{1+z_{e} l^{*} / L_{a}}{1-z_{e} l^{*} / L_{a}}\right] \approx \frac{z_{e} l^{*}}{L_{a}} .
$$

We can simplify equation 5.5 for two important cases: weak and strong absorption.

In the case of weak absorption, $l^{*} \ll L \ll L_{a}$, equation 5.5 can be approximated as 78

$$
T_{\text {weak }}(L)=\frac{l^{*}}{L} \frac{z_{e}+z_{p}}{1+2 z_{e} l^{*} / L} .
$$

In the limit of strong absorption, $l^{*} \ll L_{a} \ll L$, the transmission can be approximated as an exponential function whose decay is determined by $L_{a}$ and whose pre-factor includes the unknowns $l^{*}$ and $L_{a}$ [84]:

$$
T_{\text {strong }}(L) \approx \frac{l^{*}}{L_{a}} \frac{2\left(z_{e}+z_{p}\right)}{1+2 z_{e} l^{*} / L_{a}} \mathrm{e}^{-L / L_{a}} .
$$

In this thesis, $z_{p}$ is taken to be unity, meaning that the light is injected at a depth $l^{*}$ into the sample. We make this assumption in line with references [80, 83, 84] and [85].

\section{Extrapolation length $z_{e}$}

Key to the correct treatment of the boundaries is the dimensionless parameter $z_{e}$, the extrapolation length ratio, which can be measured experimentally from the angular distribution of the transmitted light (section 5.2.1). It can also be calculated analytically from the Fresnel reflectivities, $R$, of the interfaces in the experiment. These are defined solely by the refractive indices of the sample, its container and the air, $\eta_{s}, \eta_{c}$ and $\eta_{a}$. The cosine-angle-dependent reflectivity of an interface between two materials 1 and 2 is given by the Fresnel equation [86]

$$
R_{12}\left(\mu_{i}\right)=\frac{\eta_{1} \mu_{i}-\eta_{2} \mu_{t}}{\eta_{1} \mu_{1}+\eta_{2} \mu_{t}}
$$

Here, $i$ and $t$ denote the incident and transmitted $/$ reflected rays and $\mu=\cos \theta$. For a three-layer boundary between the sample, the container and the air, the total reflectivity is given by

$$
R_{s c a}\left(\mu_{i}\right)=\frac{R_{s c}+R_{c a}-2 R_{s c} R_{c a}}{1-R_{s c} R_{c a}}
$$


where the subscripts $s, c$ and $a$ denote sample, container and air respectively. The $n^{t h}$ reflectivity moment of the interface is given by [85]

$$
R_{n}=\int_{0}^{1}(n+1) \mu_{i}^{n} R_{s c a}\left(\mu_{i}\right) \mathrm{d} \mu
$$

and the first and second reflectivity moments make up $z_{e}$ according to reference 85

$$
z_{e}=\frac{2}{3} \frac{1+R_{2}}{1-R_{1}}
$$

In common with Kaplan et al., we choose to ignore higher order reflections in which the wall of the sample container acts as a wave guide [80]. In the case of a granular sample in water, we cannot know $\eta_{s}$. We would expect it to lie between the values of water and glass, $1.33 \leq \eta_{s} \leq 1.52$ (or $1.33 \leq \eta_{s} \leq 1.91$, in the case of BaTi glass). Taking $\eta_{s}=1.33$ gives us an estimate of $z_{e}=1.769$; but it would be preferable to measure this quantity experimentally. Our measurements of $z_{e}$ are shown in section 5.2 .1 .

\section{Experimental method}

Building on these theoretical foundations, we can obtain $l^{*}$ by measuring the transmission of a granular sample as a function of its thickness and comparing it to equations 5.5 and its simplifications for strong and weak absorption (equations 5.8 and 5.7 .

To measure $T$ for different $L$, we have five fluidised beds of thickness $L=5$ $\mathrm{mm}, 7.5 \mathrm{~mm}, 10 \mathrm{~mm}, 12.5 \mathrm{~mm}$ and $15 \mathrm{~mm}$. The height of the beds is $25 \mathrm{~cm}$ and their width $10 \mathrm{~cm}$.

We measure $T(L)$ by measuring the angularly resolved intensity of transmitted light, $I(\theta)$. This is done using the set-up shown in figure 5.2. A Nd:YAG laser with $P=5 \mathrm{~W}$ and $\lambda=532 \mathrm{~nm}$ is incident on a fluidised bed containing the granular sample. The incoming light is monitored with a beam pick-off and a control sensor. To allow for irregularities in laser intensity, the signal on the control sensor, $V_{\text {check }}(t)$, is used to adjust the intensity signal on the measurement sensor, $V(t)$. The light transmitted through the sample is detected by the measurement sensor with an integrating time of $15 \mathrm{~s}$. The sensors are small self-built circuits built around a HUV-1100BQ combined photodiode and op-amp with excellent linearity. The calibration is shown in appendix $\mathrm{D}$.

To ensure a narrow acceptance angle, a collimating lens is placed before the sensor. We calculate the acceptance angle of our optics as $4.9 \times 10^{-4} \mathrm{sr}$. The sensor and optics are mounted on a swivel arm $50 \mathrm{~cm}$ in length. The angularly resolved intensity of the transmitted light, $I(\theta)$, is measured by moving the measurement sensor in a quarter of a circle about the sample. We measure $\theta$ from $0^{\circ}$ (the surface normal) to $90^{\circ}$ in steps of $5^{\circ}$. A black screen ensures that no refections 
enter the transmission hemisphere and the experiment is performed under blackout conditions.

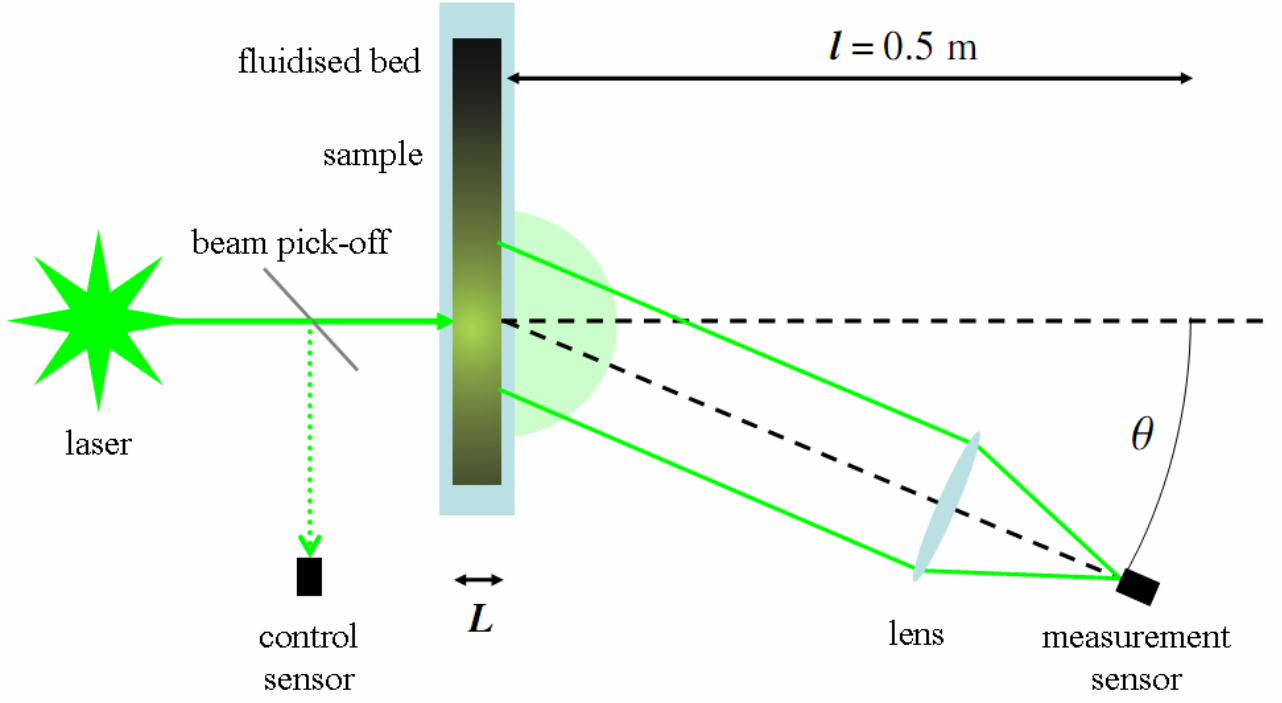

Figure 5.2.: Experimental set-up to measure $l^{*}$ in transmission, viewed from above. A laser beam is normally incident on a fluidised bed of thickness $L$ and width $10 \mathrm{~cm}$ filled with the granular sample. The transmitted light is detected by the measurement sensor, which is mounted on an arm that can be moved through the angles $0 \leq \theta \leq 90^{\circ}$. A lens is placed in front of the sensor to collimate the light. A beam pick-off allows the intensity of the incident laser beam to be monitored by the control sensor.

$I(\theta)$ divided by the acceptance angle of the detector gives the transmitted flux, $\Phi$, as a function of $\theta . \Phi(\theta)$ measured over a quarter of a circle is integrated over the transmission hemisphere to give the total transmitted flux [87]

$$
\Phi_{T}=\int_{0}^{\pi} \int_{0}^{\pi / 2} \Phi(\theta) \sin \theta \mathrm{d} \theta \mathrm{d} \phi=\pi \int_{0}^{\pi / 2} \Phi(\theta) \sin \theta \mathrm{d} \theta .
$$

The transmitted flux divided by the incoming flux measured by the control sensor gives the transmissivity, $T$. In making this calculation, we correct $\Phi$ to take account of the lens transmissivity, which we measured to be 0.91 for $\lambda=532 \mathrm{~nm}$. Measuring $T$ for different sample thicknesses, $L$, and fitting $T(L)$ with equation 5.5 and its simplifications for strong and weak absorption (equations 5.8 and 5.7 ) gives us $l^{*}$. Our results are shown in section 5.2.1.

\subsubsection{Measuring $l^{*}$ by coherent backscattering}

Coherent backscattering is an interference effect that leads to a cone-shaped enhancement of the backscattered intensity profile, $B(\theta)$, in a small angular range 
around the normal, $\theta=0[82]$. The width of the cone is a measure for $l^{*}$. Consider the multiply-scattering medium sketched in figure 5.3(a). Due to time reversal symmetry, each scattering path has a counter-propagating partner. Because the path lengths are by definition identical, the light on this pair of paths interferes constructively. At $\theta=0^{\circ}$, the enhancement factor is two (figure 5.3(b)) [88, 82].
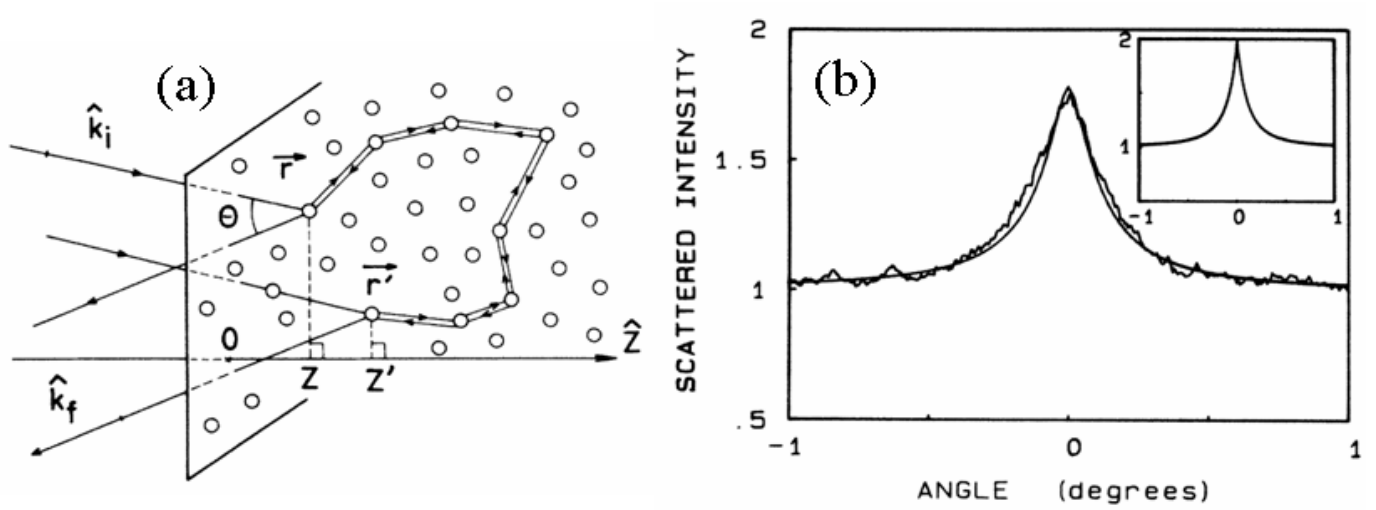

Figure 5.3.: (a) A light path and its time-reversal symmetric partner propagating through a scattering medium. (b) Experimental and (inset) theoretical coherent backscattering cones for a suspension of $0.46 \mu \mathrm{m}$ polystyrene beads in water [89]. The experimental peak is not at 2 because absorption, finite sample thickness and localisation reduce the contribution of long-path photons [82. Both images taken from reference 88 .

Zhu et al. relate the full width at half-maximum of the backscattered cone, $W$, to $l^{*}$ according to 90 ]

$$
W^{-1}=\left(\frac{2}{3}+z_{e}\right) k l^{*},
$$

where $k=2 \pi / \lambda$ is the incident wave number.

Our experimental work on coherent backscattering was done in collaboration with S. Fiebig at the University of Konstanz. Figure 5.4 shows the set-up we use to measure the backscattering cone of our granular samples. The sample is placed into a fluidised bed $4 \mathrm{~cm} \times 4 \mathrm{~cm}$ in cross-section and $25 \mathrm{~cm}$ in height. A laser of $\lambda=575 \mathrm{~nm}$ and $P=2.7 \mathrm{~W}$ passes first through a spatial filter to increase the beam diameter to $5 \mathrm{~mm}$. The beam is then passed through a beam splitter and is normally incident on the sample. Single reflections are removed from the backscattered light by a $\lambda / 4$ plate and polariser. The backscattered light, after passing once more through the beam splitter, is focused by the lens onto a 2048 $\times 2048$ pixel CCD. The CCD has a pixel side length of $7.4 \mu \mathrm{m}$.

Our results are shown in section 5.2.2. 


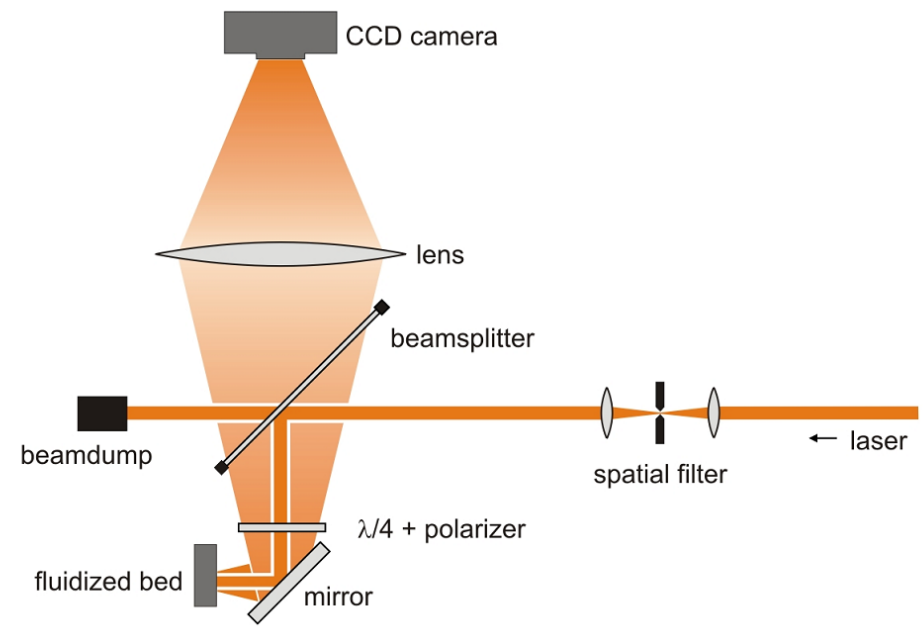

Figure 5.4.: Set-up to measure coherent backscattering on a granular sample in a fluidised bed. The CCD chip measures $15 \mathrm{~mm} \times 15 \mathrm{~mm}$ with a resolution of $2048^{2}$ pixels. The pixel side length is $7.4 \mu \mathrm{m}$. The lens has a focal length of $50 \mathrm{~cm}$. The laser has $\lambda=575 \mathrm{~nm}$ and $2.7 \mathrm{~W}$ power. Image property of S. Fiebig 91 .

\subsubsection{Measuring $l^{*}$ by incoherent backscattering}

Snabre and Arhaliass [92] suggest a means of measuring $l^{*}$ by looking at the backscattered light away from the coherent cone. From the radial surface flux profile, $F_{s}(\rho)$, which is shown in figure 5.5, the transport mean free path can be obtained according to two relationships, which describe the short-path photons (left of the kink) and the long-path photons (right of the kink). $\rho$ is the radial distance from the centre of the backscattered spot (where the laser is incident). The position of the kink is at $\rho=4 l^{*}$.

For short photon paths, $\rho<4 l^{*}$, the surface flux is given by 92

$$
F_{s}(\rho)=\frac{4^{-1.6}}{\pi \rho^{2}}\left(\frac{\rho}{l^{*}}\right)^{0.6}
$$

For short paths, the diffusion approximation does not hold: light is transported ballistically. In this regime, absorption can be neglected. For long photon paths $\left(\rho>4 l^{*}\right)$, absorption can no longer be neglected, and we return to the diffusive model of light transport. Here, $F_{s}$ is a function of $\rho$ and $L_{a}$. In this case, the appropriate expression is 92

$$
F_{s}\left(\rho, L_{a}\right) \approx \frac{l^{*}}{\pi \rho^{3}} \exp \left[\frac{-1.3 \rho}{l^{*}}\left(\frac{L_{a}}{l^{*}}\right)^{-1 / 2}\right] .
$$

The incoherently backscattered spot is measured using the set-up shown in figure 5.6. A Nd:YAG laser with $\lambda=532 \mathrm{~nm}$ and $P=1 \mathrm{~W}$ is normally incident 


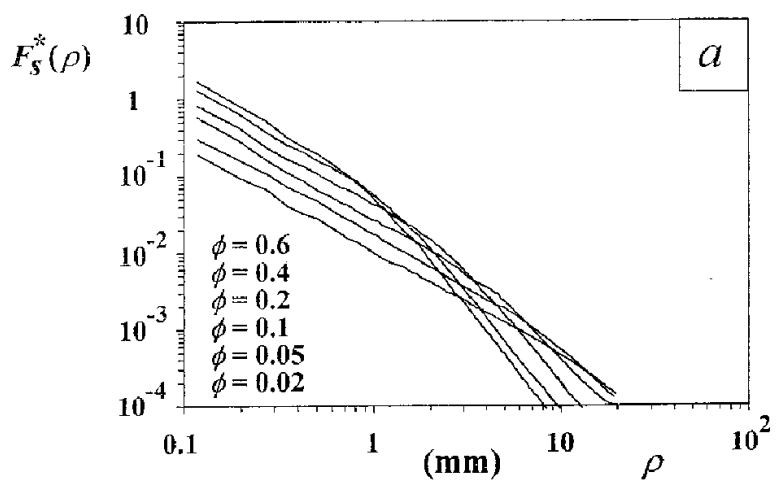

Figure 5.5.: The radial flux profile of backscattered light from $46 \mu \mathrm{m}$ alumina particles in water. Two regimes are visible: left of the kink, the short-path photons make up the flux. The flux to the right of the kink is made up of long-path photons. Figure taken from reference 92 .

on the sample in a fluidised bed of thickness $L=12.5 \mathrm{~mm}, 10 \mathrm{~cm}$ width and 25 cm height. A black-and-white CCD camera with 1 Megapixel and 8-bit depth equipped with a $28 \mathrm{~mm}$ Olympus objective, is mounted a distance of $1 \mathrm{~m}$ from the sample and at an angle of $2.5^{\circ}$ to the optical axis. A way of working around the limited bit depth of the camera would be to photograph the spot for a number of different exposure times. The intensity profiles, $I(\rho)$, could then be overlaid to obtain a greater effective intensity range.

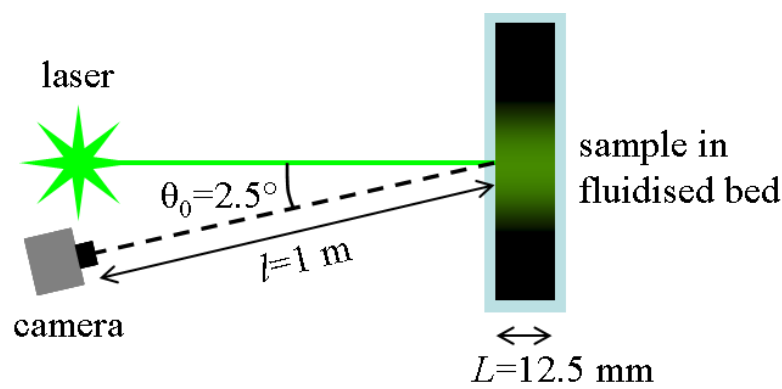

Figure 5.6.: Set-up for measuring $l^{*}$ by incoherent backscattering. View from above. The backscattered laser spot is photographed by a camera mounted a few degrees off the optical axis.

\subsection{Results}

In all our experiments, our granular sample is an agitated packing of glass spheres in a water fluidised bed. Performing measurements on fluidised packings allows 
us to control the volume fraction, $\phi$, whilst at the same time blurring out the laser speckle pattern that would otherwise obscure our results. ${ }^{4}$

\subsubsection{Transmission results}

These measurements are made on the unetched small and large Mo-Sci soda-lime glass spheres that we used in part $\mathrm{I}$ of this thesis $(\eta=1.52)$ and on BaTi glass spheres of mean diameter $140 \mu \mathrm{m}(\eta=1.91)$. Five fluidised beds of thickness $L=5 \mathrm{~mm}, 7.5 \mathrm{~mm}, 10 \mathrm{~mm}, 12.5 \mathrm{~mm}$ and $15 \mathrm{~mm}$ are used to measure $T(L)$ in the set-up described in figure 5.2 .

\section{Results for $Z_{e}$}

In section 5.1.1, we show how the extrapolation length ratio can be calculated for boundaries between media of known refractive index. For a glass tank in air filled with water, $z_{e}=1.769$.

We do not know the refractive index of a packing of glass spheres in water. We would expect it to lie between the values of water and glass, $1.33 \leq \eta_{s} \leq 1.54$. We could simply take $z_{e}$ for a tank of water, but here we try to improve on this by measuring $z_{e}$ experimentally. For this, we measure the angular distribution of the diffusively transmitted light, $I(\theta)$, and calculate $z_{e}$ from a fit to the experimental data in the following way.

The cosine-angle-dependent intensity, $I(\mu)$, is related to the probability distribution, $P(\mu)$, according to 85 ]

$$
P(\mu)=\frac{1}{N} I(\mu)
$$

where $\mu=\cos \theta$ and the normalisation factor, $N$, is obtained by

$$
N=\int_{0}^{\pi / 2} I(\theta) \sin \theta \mathrm{d} \theta
$$

Diffusion theory tells us that the normalised probability distribution is given by [85]

$$
\frac{P(\mu)}{\mu}=\frac{z_{e}+\mu}{1 / 2 z_{e}+1 / 3} .
$$

We can fit equation 5.19 to our measured $P(\mu) / \mu$. This is shown in figure 5.7 . The fit parameter is $\eta_{s}$, the refractive index of the sample, which appears in the equation implicitly through the value of $z_{e}$, as described in section 5.1.1. For the small Mo-Sci grains, we obtain $\eta_{s}=1.38$ and $z_{e}=1.95$, both very reasonable

\footnotetext{
${ }^{4}$ The speckle pattern is necessary to measure the time scale of granular movement, $\tau$. When measuring $l^{*}$, however, it just gets in the way.
} 
values. For the large Mo-Sci grains, the fit yields $z_{e}=2.98$ and $\eta_{s}=1.63$. This value for $z_{e}$ is unphysical, since it exceeds the range $1.33 \leq \eta_{s} \leq 1.52$. For this reason, we use the theoretical value $z_{e}=1.769$, in common with [84].

A close look at figure 5.7 reveals that, at small $\mu$ (large $\theta$ ), there is very little difference between the two fits, even though one gives a plausible $\eta_{s}$ and the other one does not. The method may well yield better results if the error bars at small $\mu$ could be reduced, say, by increasing integration time; and by measuring at smaller intervals in the range $0<\mu<0.5$. If this leads to sufficient improvement, a measured value for $z_{e}$ is to be preferred over one calculated for $\eta_{s}=\eta_{\text {water }}$.

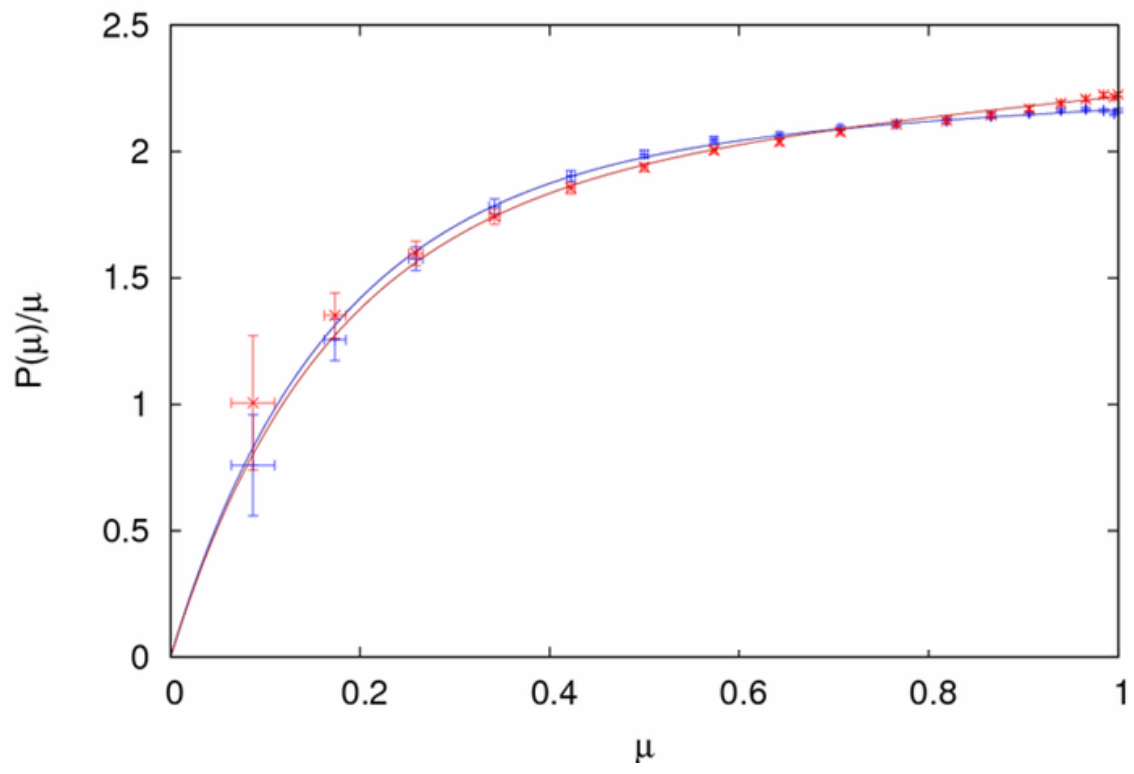

Figure 5.7.: $\quad P(\mu) / \mu$ for Mo-sci grains measured with $L=10 \mathrm{~mm}$. The lines are fits using equation 5.19 using $\eta_{s}$ as the fit parameter. For the large Mo-Sci grains (blue), the fit yields $\eta_{s}=1.63$ and $z_{e}=2.98$; for the small Mo-Sci (red), $\eta_{s}=1.38$ and $z_{e}=1.95$.

Given our assumption that $z_{p}=1$ and our calculated value of $z_{e}$, we see that we have a framework for measuring $l^{*}$.

\section{Weak or strong absorption limit?}

We measure $T(L)$ for five sample widths: $5 \mathrm{~mm}, 7.5 \mathrm{~mm}, 10 \mathrm{~mm}, 12.5 \mathrm{~mm}$ and $15 \mathrm{~mm}$. The grains are BaTi glass spheres of diameter $140 \mu \mathrm{m}$. The illumination is laser light with $P=5 \mathrm{~W}$ and $\lambda=532 \mathrm{~nm}$. The driven granular bed has a volume fraction of $0.60 \pm 0.01$. To find out if our system is in the weak or strong absorption limit, we fit equation 5.5 and its simplifications for strong and 
weak absorption (equations 5.8 and 5.7) to the measurement data. We take fixed values of $z_{p}=1$ and $z_{e}=1.769$. The results are shown in figure 5.8 .

Weak absorption is such a poor match for the data that we reject the simplification out of hand. The strong absorption fit lies on the data very well, but it yields an implausibly large value $l^{*} \sim 100 \mathrm{~km}$. Fitting $T_{\text {strong }}(L)$ with $l^{*}$ fixed at $1.34 \mathrm{~mm}$ gives the very reasonable value $L_{a}=2.45 \mathrm{~mm}$. This test shows that the function $T_{\text {strong }}(L)$ is insufficiently sensitive to $l^{*}$ to be of use. A fit with the full solution

$$
T(L)=\frac{\sinh \left(z_{p} l^{*} / L_{a}+\epsilon\right) \sinh (\epsilon)}{\epsilon \sinh \left(L / L_{a}+2 \epsilon\right)}
$$

with

$$
\epsilon=\frac{z_{e} l^{*}}{L_{a}}
$$

yields an excellent fit with the length scales $l^{*}=1.34 \mathrm{~mm}$ and $L_{a}=2.25 \mathrm{~mm}$. Both values compare well to literature values (table 5.3).

For our experiment, $l^{*} \sim L_{a}$, so it would appear that we are in an intermediate absorption range where neither simplification holds. This is certainly the reason that $T_{\text {weak }}(L)$ fails to describe our experimental data. It is hard to say if this is the reason that $T_{\text {strong }}(L)$ fails, or if its failure is due to the small number of data points. In any case, the full solution for $T(L)$ (equation 5.5) is to be preferred over either simplification.

\section{$T(L)$ for different $\phi$}

It is to be expected that increasing the volume fraction of the granular sample will reduce $l^{*}$, since it decreases the distance between grains and thence scattering events. Figure 5.9 shows $T(L)$ measured for three different volume fraction $\$^{5}$ and fitted with equation 5.5. The data for $\phi=0.60$ are the same as in the previous section. The fit parameters are $l^{*}$ and $L_{a}$, and these are given in table 5.2.1.

As expected, $l^{*}$ is greater at lower $\phi$. We see that $L_{a}$ also increases with decreasing $\phi$. This is a consequence of the greater distance between absorbtion sites, BaTi glass being more strongly absorbing than water. From section 5.1.1, we recall that $L_{a}=\sqrt{l_{a} l^{*} / 3}$, where $l_{a}$ is a material constant describing the attenuation of light through the sample material [82]. This quantity is also given in table 5.2.1. It is almost independent of volume fraction, but not quite: at lower $\phi, l_{a}$ is also slightly smaller. This slight trend is readily explained: $l_{a}$ is a material parameter for the whole sample, which is a mixture of water and glass. As we have already observed, water is less absorbing than BaTi glass, so an effective

\footnotetext{
${ }^{5}$ Due to incomplete calibration, there is an additional systematic error on all $\phi$ of an estimated \pm 0.02 .
} 


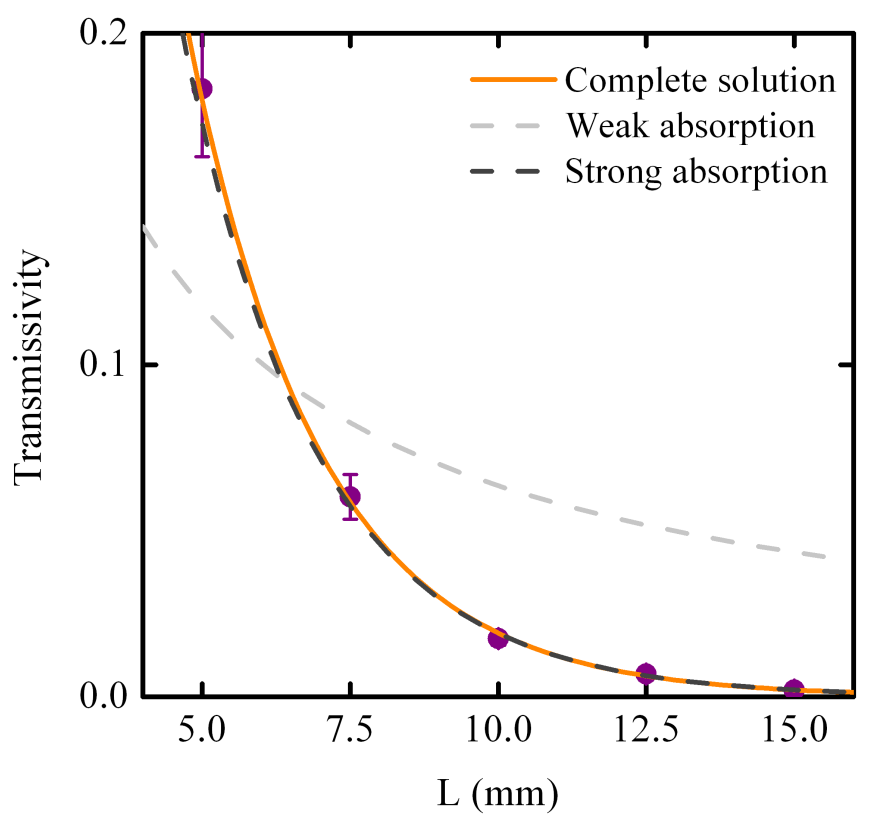

Figure 5.8.: $T(L)$ for a driven packing of BaTi glass spheres $140 \mu \mathrm{m}$ in diameter, with $\phi \approx 0.60$. The light grey dashed line is a fit with equation 5.7 for weak absorption, yielding $l^{*}=0.5 \mathrm{~mm}$. The dark grey dashed line is for strong absorption, (equation 5.8), giving $l^{*} \sim 100 \mathrm{~km}$ and $L_{a}=2.3 \mathrm{~mm}$. The orange line is a fit to the full solution (equation 5.5), which gives $l^{*}=1.34 \mathrm{~mm}$ and $L_{a}=2.25 \mathrm{~mm}$. The fit parameters are $l^{*}$ and $L_{a}$, where appropriate. We use $z_{p}=1$ and $z_{e}=1.769$.

reduction in the amount of glass in the mixture should reasonably be expected to bring down the attenuation of the mixture by a small amount.

The values we obtain for $l^{*}$ and $L_{a}$ compare very well to literature results (section 5.3). Our experimental results for the small Mo-Sci grains can be found section 5.3 and in table 5.3 .

An interesting observation can be made from the transmissivity at $L=5 \mathrm{~mm}$ and $\phi=0.50$. The measurement point is not as well described by the fit as for greater sample thickness. For small thickness, the multiple-scattering condition $l^{*} \ll L$ no longer holds. Consequently, the diffusion approximation is no longer valid for our measurements at small $L$. Astonishingly, the model still seems to fit the data well even for $L=5 \mathrm{~mm}$ for the two denser samples.

\subsubsection{Coherent backscattering results}

Our experimental work on coherent backscattering was done in collaboration with S. Fiebig at the University of Konstanz. Let us start this section by estimating 


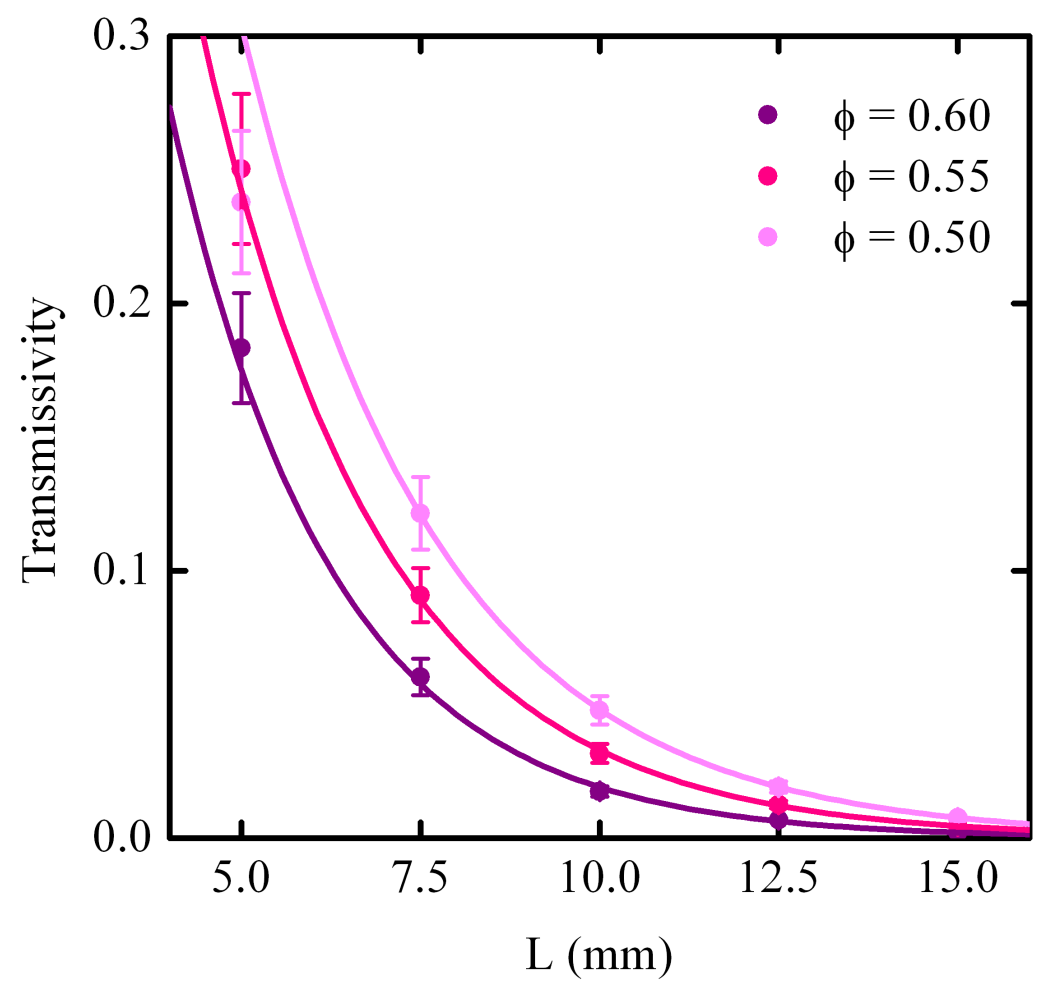

Figure 5.9.: $\quad T(L)$ for a driven packing of BaTi glass spheres $140 \mu \mathrm{m}$ in diameter, for three different volume fractions $0.50 \leq \phi \leq 0.6$. The lines are fits to equation 5.5 .

our experimental resolution. Equation 5.14 (repeated below) relates $l^{*}$ to the full width at half maximum of the backscattered cone, $W$ [90]

$$
W^{-1}=\left(\frac{2}{3}+z_{e}\right) k l^{*}
$$

If we assume we can resolve a $W$ of five pixel lengths, we can calculate the maximum transport mean free path, $l_{\max }^{*}$, detectable by our instrument. On our CCD, five pixel lengths correspond to $37 \mu \mathrm{m}$. We have $\lambda=575 \mathrm{~nm}$ and we take our calculated value of $z_{e}=1.769$ (section 5.1.1). This tells us that we can resolve $l^{*}$ at lengths $l^{*}<1.01 \mathrm{~mm}$. Our measurements of $l^{*}$ in transmission are of this order, so it is not certain from this estimate whether the coherent backscattering method is suitable.

The true resolution of the experiment is determined by the size of the laser spot seen by the CCD when the sample is replaced by a mirror. This is much poorer than the theoretical resolution, as shown in figure 5.10, Repeating our 


\begin{tabular}{|c|c|c|c|c|c|}
\hline$\phi$ & $l^{*}(\mathrm{~mm})$ & $l^{*}(d)$ & $L_{a}(\mathrm{~mm})$ & $L_{a}(d)$ & $l_{a}(\mathrm{~mm})$ \\
\hline 0.60 & 1.3 & 9 & 2.3 & 16 & 11.2 \\
0.55 & 1.7 & 12 & 2.5 & 18 & 11.0 \\
0.50 & 2.0 & 14 & 2.7 & 19 & 10.9 \\
\hline
\end{tabular}

Table 5.1.: The length scales obtained from $T(L)$ for three different volume fractions of BaTi glass grains. $d=140 \mu \mathrm{m}$ is the mean grain diameter. We estimate the error on $l^{*}$ to be $\pm 22 \%$ and on $L_{a}$ to be $\pm 3 \%$.

previous calculation for $W=200 \mu \mathrm{m}$ - a rather generous estimate based on the width of the laser spot in figure 5.10 gives an estimate of $l_{\max }^{*}=180$ $\mu \mathrm{m}$. This corresponds to little more than one particle diameter. This calculation suggests that is is not possible to resolve $l^{*}$ for our samples with the coherent backscattering method.

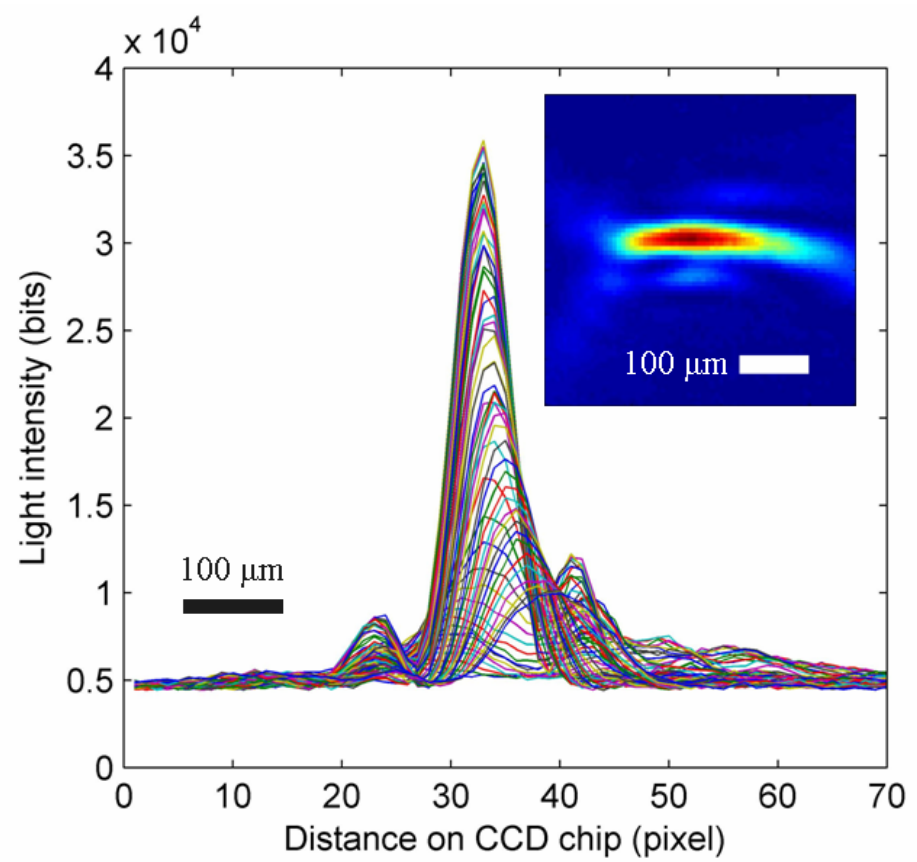

Figure 5.10.: Testing the true experimental resolution from the image of the laser spot on the CCD. Inset: the image on the CCD, intensity in bits. The "spot" is more of a banana shape. The graph shows the intensity profiles of vertical cuts through the laser spot image.

The backscattering cone indeed proves too small for us to resolve. Figure 5.11(a) shows our backscattered intensity profile, averaged over 100 images, each with an exposure time of $0.5 \mathrm{~s}$. The grains are soda-lime glass spheres $125 \mu \mathrm{m}$ in diameter from Whitehouse Scientific and the flow rate through the fluidised bed is $Q=600 \mathrm{ml} / \mathrm{s}, \phi \approx 0.50$. Figure 5.11(b) is the radial average of the backscattered 
pattern. We would expect to see the enhanced backscattering on the scale of $<40 \mu \mathrm{m}$. There is clearly nothing of this nature occurring in the figure. Both the graph and the inset show that our backscattered profile is dominated by glory rings (single-scatterer interference). It is possible that some tuning of the spatial filter would decrease the size of the laser spot, and that and increasing the distance between the sample and the camera might also contribute to an improved experimental resolution. However, it is unlikely that it will improve things enough to measure $l^{*}$ of the magnitude we expect.

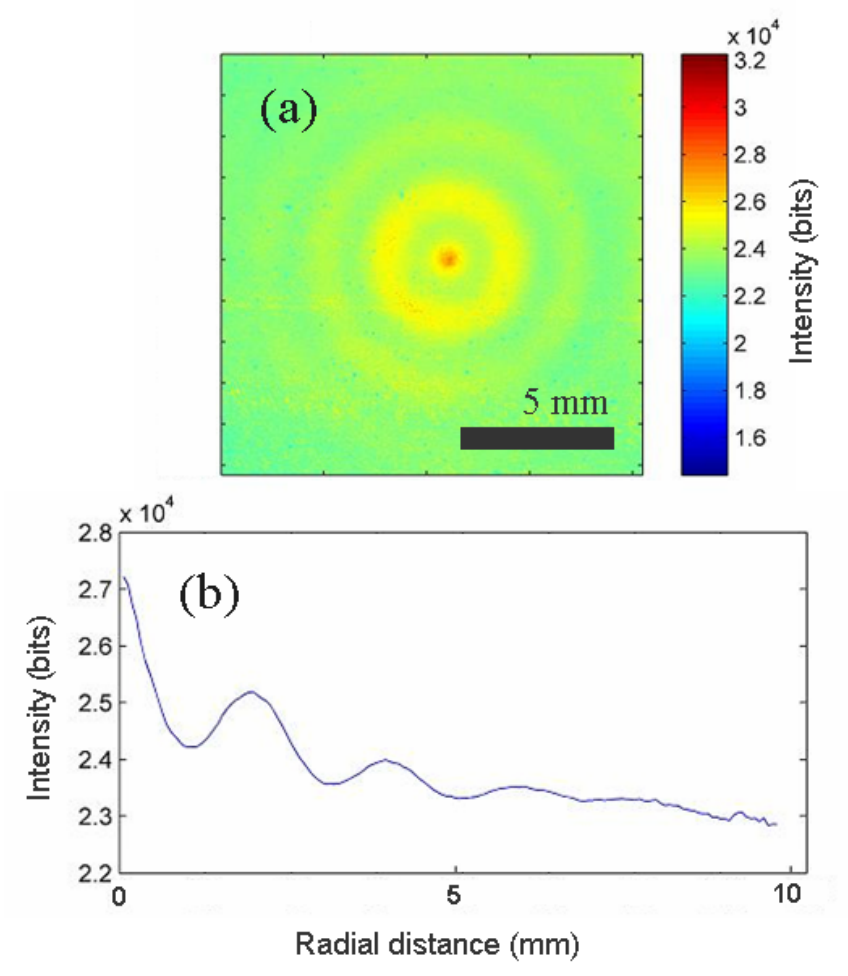

Figure 5.11.: The backscattering intensity patterns in bits (a) seen by the CCD (axis unit is pixel) and (b) as a radial average. The rings are glory.

\subsubsection{Incoherent backscattering results}

The experiments shown in this section are performed with the same BaTi glass spheres and the $L=12.5 \mathrm{~mm}$ fluidised bed used for the transmission measurements in section 5.2.1. Again, we start with some predictions.

\section{Prediction of backscattered spot}

From Mie theory (predictions in appendix E), we can use equations 5.15 and 5.16 make predictions about the backscattered spot using the method of reference [92]. 
The surface flux, $F_{s}$, as a function of radial distance from the centre of the spot, $\rho$, is calculated for small Mo-Sci grains (figure 5.12). Here, volume fraction is varied through $\phi=0.45,0.5,0.55,0.6$ and 0.65 . Our calculations show that it should be experimentally possible to discriminate between samples of different volume fraction as long as experimental noise is low.

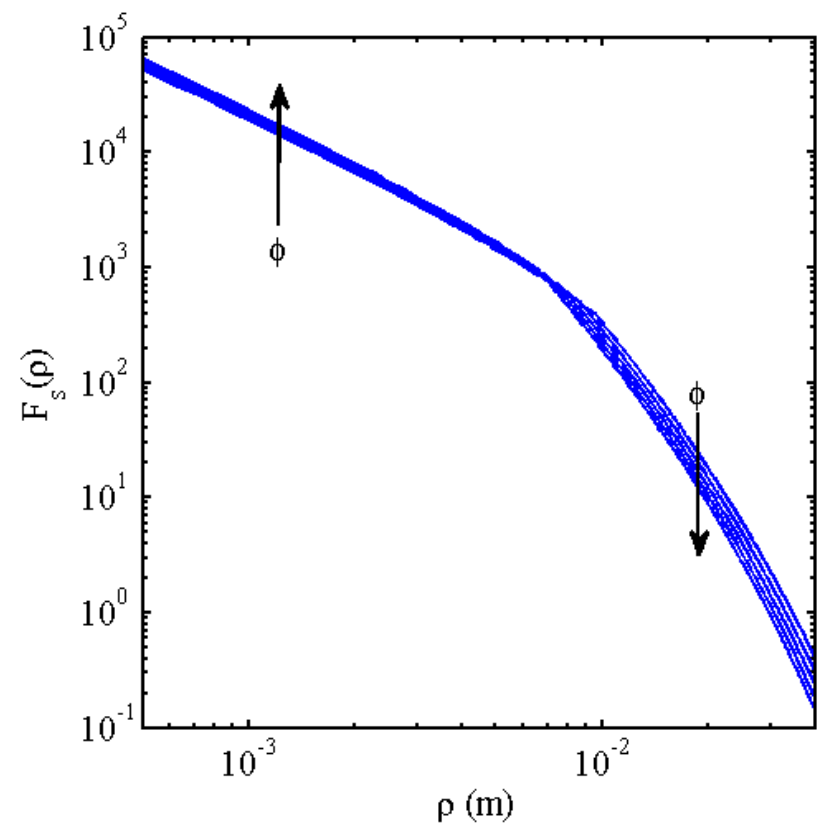

Figure 5.12.: $\quad$ Surface flux of backscattered light as a function of radial distance from the centre of the spot. Predicted curves for small Mo-Sci grains with $\phi=0.45$, $0.5,0.55,0.6$ and 0.65 .

Some preliminary experimental results are shown in figure 5.13 . Experiments were made immediately after the $T(L)$ measurements of section 5.2 .1 for $L=12.5$ $\mathrm{mm}$, for the same three volume densities $\phi=0.60,0.55$ and 0.50 .6 The sample is BaTi glass spheres $140 \mu \mathrm{m}$ in diameter. The inset shows the backscattered spot at $\phi=0.55$. The graph shows the radially averaged intensity profile of the spots for the three different volume fractions. We see that we can indeed discriminate between different volume fractions, and this shows that the method has potential. However, we also see that glory is strongly visible, which could well obscure the physics we are trying to see.

\footnotetext{
${ }^{6}$ Due to incomplete calibration, there is an additional systematic error on all $\phi$ of an estimated \pm 0.02 .
} 


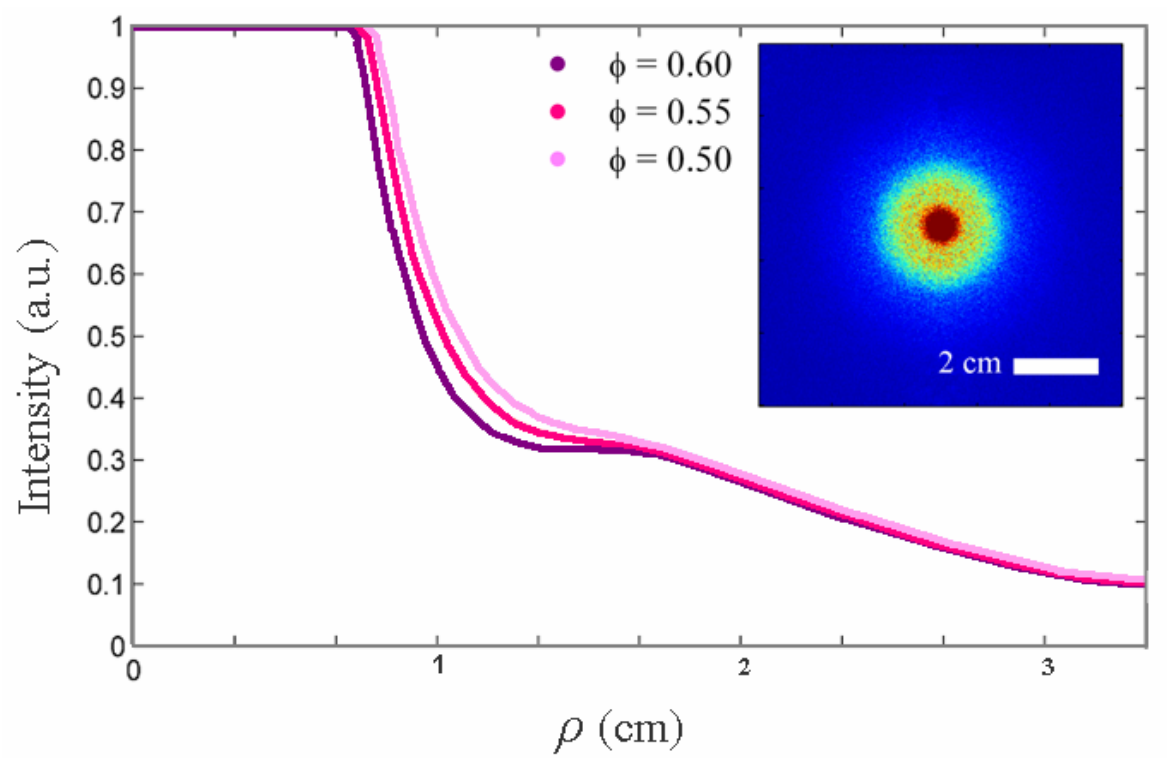

Figure 5.13.: Radially averaged intensity profiles of the incoherently backscattered spot for three volume fractions. $\phi$ is known with an accuracy of \pm 0.01 . Inset: the backscattered spot of at $\phi=0.55$. The intensity scale is logarithmic in order better to show the details. Grains are BaTi glass spheres of $140 \mu \mathrm{m}$ diameter. The exposure time is $100 \mathrm{~ms}$.

\subsection{Discussion}

In this chapter, we have measured $l^{*}$ and $L_{a}$ using three different experimental methods, and met with varying success. In this section, we evaluate our experimental results: first, in terms of theoretical predictions and literature results, then, in terms of $T_{\text {kin }}$.

\section{Theoretical prediction of $l^{*}$}

Given perfect spheres, we can calculate $l^{*}$ directly from the calculated Mie scattering functions [93, 94]. The transport mean free path is calculated as $l^{*}=$ $(2 R) /(3 \phi(1-\langle\cos \theta\rangle))$, where $\phi$ is the packing fraction, $R$ the particle radius and $g \equiv\langle\cos \theta\rangle$ is the scattering asymmetry. $g$ tends to zero in the isotropic scattering limit. We calculate $g$ in appendix E.

Figure 5.14 shows calculations of $l^{*}$ in units of particle diameter as a function of packing fraction for small and large Mo-Sci grains. Also shown is the prediction for $1 \mu \mathrm{m}$ polystyrene latex spheres, an isotropic scatterer often used for calibrations. The triangle is the experimental value obtained by the transmission method for the small Mo-Sci grains (section 5.2.1). The experimental $l^{*}$ is smaller than the calculated values; this is because real grains are not perfectly smooth or spherical. Asperities and asphericity lead to a reduction in the mean scattering 
asymmetry, which means that fewer scattering events are necessary to randomise the light paths. This has the effect of making $l^{*}$ smaller. Leutz and Rička made the same comparison for their samples and they also observed $l_{\text {Mie }}^{*}>l_{\text {experiment }}^{*}$ [84].

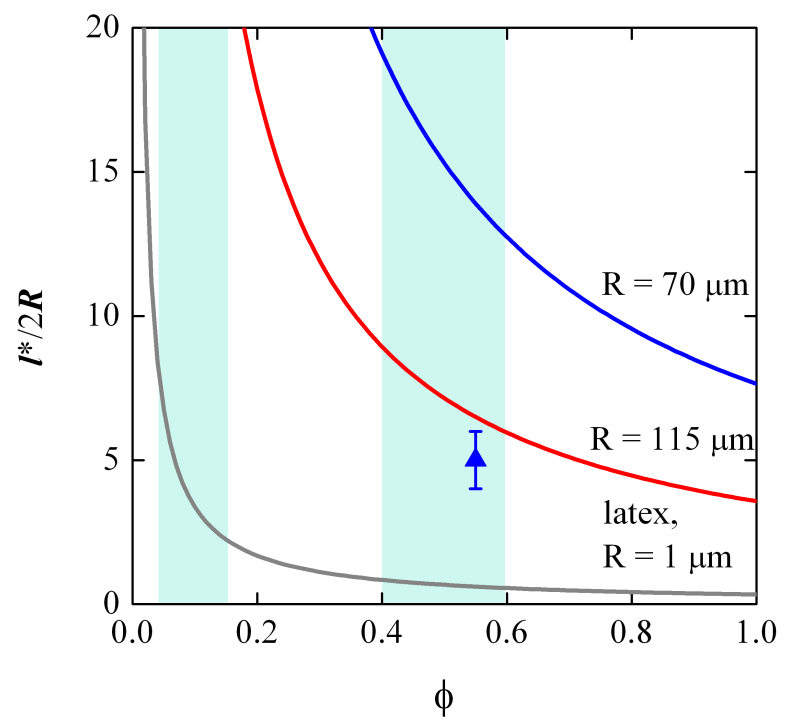

Figure 5.14.: $l^{*}(\phi)$ calculated for certain types of particle. Blue: soda lime spheres of $R=70 \mu \mathrm{m}$ in water (small Mo-Sci); red: soda lime spheres of $R=115 \mu \mathrm{m}$ in water (large Mo-Sci); and grey: latex spheres of $R=1 \mu \mathrm{m}$ in water, such as are often used for calibration measurements. The blue shaded regions show the experimentally relevant volume densities. The blue triangle is measured experimentally in transmission on small Mo-Sci grains (section 5.2.1).

\section{Comparison of results}

A brief tabular comparison of $l^{*}$ and $L_{a}$ measured or calculated by various methods is made in table 5.3. All the results represented in the table are for granular packings or suspensions. The experimental ("E") and numerical ("N") data from five other studies and our own experimental and theoretical ("T") results are almost all in the range $4 d<l^{*}<40 d 7$ This is very encouraging, but the table allows us more than just an order-of-magnitude comparison.

From the table, we observe that granular packings in air have a consistently shorter transport mean free path than those in water; this is because a greater mismatch of refractive index leads to less anisotropy in the scattering probability, meaning that fewer scattering events are necessary to randomise the light paths.

\footnotetext{
${ }^{7}$ We take the liberty of ignoring the $l^{*}=100 \mathrm{~km}$ result in this discussion!
} 
Our own experimental results do not seem to bear this out: with $\eta_{s-l}=1.52$, the index of refraction of soda-lime glass is much closer than BaTi glass $\left(\eta_{B a T i}=1.91\right)$ to that of water $\left(\eta_{\text {water }}=1.33\right)$. The BaTi grains are less closely matched to water, which we would expect to lead to a smaller $l^{*}$ : this is unfortunately not what we observe. The comparison of $l^{*}$ for soda-lime and BaTi glass must be made with caution, since $\phi$ was not measured for the soda-lime grains. It is possible that the soda-lime grains have a greater surface roughness than the BaTi grains, which could lead to this unexpected result. Another contributing factor could be that the calculated value of $z_{e}=1.769$ is a poor description of the boundary conditions for the BaTi grains.

We have already remarked that the theoretical predictions yield a greater $l^{*}$ than experiment, and that this is due to the asperities and asphericity of real grains. The study of Snabre and Arhaliass uses alumina particles obtained by grinding, which are not at all close to being smooth or spherical [92]. Here, then, we again see the effect of surface roughness and asphericity on the transport mean free path: the more a grain departs from the smoothly spherical, the shorter is $l^{*}$ in a packing of these grains.

The transmission data from Leutz and Rička yield consistently greater $l^{*}$ than all the other studies shown in the table [84]. It is hard to tell how this observation should be interpreted, since they use the approximation for strong absorption, which is valid in the limit $l^{*} \ll L_{a} \ll L$, yet their results say that $l^{*}>L_{a}$.

\section{Granular temperature reprieved}

Our motivation for measuring $l^{*}$ was to be able to combine this with speckle measurements (DWS) to obtain a measure for the kinetic granular temperature, $T_{\text {kin. }}$. Our measurements of $l^{*}$ can be used, together with the measurement of the speckle decorrelation time $\tau$ presented in chapter 4 , to estimate the kinetic granular temperature, $T_{\text {kin }}$.

In figure 4.4. $(1 / \tau)^{2}$ as a function of $\phi$ is measured for the large Mo-Sci grains. We can use this measurement at $\phi=0.55$ together with $l^{*}=5 d$ measured in transmission for the same grains, to estimate of the mean velocity of the grains, $\langle\vec{v}\rangle=6 \mathrm{~mm} / \mathrm{s}$. This is in good agreement with visual inspection of the granular bed in motion. Using the same quantities, we calculate $T_{\text {kin }} \propto$ $m\left(l^{*} / \tau\right)^{2}=3.4 \times 10^{-5} \mathrm{~J}$, where $m$ is the mass of the grain. Before we compare these estimates to literature results, a few cautionary words. First, $T_{\text {kin }}$ is related to $l^{*}$ and $\tau$ by proportionality. There is a constant of proportionality of order 1 that accounts for the optics of our set-up that we have not measured and therefore neglect in our calculation. Second, the value we take for $l^{*}$ is measured for an unknown volume fraction; we take $\phi=0.55$ as a reasonable estimate. Third, $\tau$ and $l^{*}$ are measured in two different fluidised beds driven by different flow rates, $Q$. With these warnings in mind, we venture to compare our $\langle\vec{v}\rangle$ and $T_{\text {kin }}$ to the results of others. 


\begin{tabular}{|c|c|c|c|c|c|}
\hline Author & Sample & & Method & $l^{*}(d)$ & $L_{a}(d)$ \\
\hline Crassous [79] & soda-lime/air & $\mathrm{E}$ & strong & $4.1 \pm 0.5$ & $10.0 \pm 0.25$ \\
" & soda-lime/air & $\mathrm{N}$ & ray tracing & 3.3 & - \\
soda-lime/water & $\mathrm{N}$ & ray tracing & 17 & - \\
Leutz \& Rička [84] & $180 \mu \mathrm{m}$ glass/water & $\mathrm{E}$ & strong & $37.7 \pm 0.5$ & $9 \pm 1$ \\
" & $225 \mu \mathrm{m}$ glass/water & $\mathrm{E}$ & strong & $36.8 \pm 0.8$ & $10 \pm 1.3$ \\
Menon \& Durian [66] & $380 \mu \mathrm{m}$ glass/water & $\mathrm{E}$ & strong & $22.1 \pm 0.8$ & $7 \pm 1$ \\
Snabre \& Arhaliass [92] & polystyrene/air & $\mathrm{E}$ & strong & 7.5 & 17 \\
Snabre \& Crassous [95] & alumina/water & $\mathrm{E}$ & incoherent & $2 \pm 1$ & not stated \\
\hline Us & glass/water & $\mathrm{E}$ & strong & $8.0 \pm 0.4$ & $41.2 \pm 0.6$ \\
" & soda-lime/water & $\mathrm{E}$ & full & $5 \pm 1$ & $19.2 \pm 0.6$ \\
" & Soda-lime/water, $\phi=0.55$ & $\mathrm{~T}$ & Mie theory & 13.9 & - \\
" & BaTi/water, $\phi=0.55$ & $\mathrm{E}$ & weak & $3 \pm 1$ & - \\
" & BaTi/water, $\phi=0.55$ & $\mathrm{E}$ & strong & $100 \mathrm{~km}$ & 16.4 \\
& BaTi/water, $\phi=0.55$ & $\mathrm{E}$ & full & $12 \pm 2.5$ & $17.9 \pm 0.5$ \\
& BaTi/water, $\phi=0.50$ & $\mathrm{E}$ & full & $14 \pm 3$ & $19.4 \pm 0.6$ \\
& BaTi/water, $\phi=0.60$ & $\mathrm{E}$ & full & $9 \pm 2$ & $16.1 \pm 0.5$ \\
\hline
\end{tabular}

Table 5.2.: Literature values and our own results for $l^{*}$ and $L_{a}$ (where appropriate). All samples are granular packings or suspensions; the length unit is $d$, the mean particle diameter. E, N and T stand for experiment, numerics and theory. Under method we refer to the following: full means $T(L)$ fitted with the full solution (equation 5.5). Strong means $T(L)$ fitted with the approximation for strong absorption (equation 5.8). Mie theory refers theoretical values obtained using the method of Bohren and Huffman and Mätzler [93, 94] (appendix E). Incoherent means using incoherent backscattering (section 5.1.3).

Menon and Durian measure $T_{\text {kin }}$ of glass grains flowing through a chute, using DWS [66]. They obtain $\langle\vec{v}\rangle \approx 1 \mathrm{~mm} / \mathrm{s}$ and $T_{\text {kin }} \approx 10^{-6} \mathrm{~J}$.

Losert et. al measure $T_{\text {kin }}$ by particle tracking in a shaken monolayer of spheres. From their results, we calculate $3<\langle\vec{v}\rangle<12 \mathrm{~cm} / \mathrm{s}$ and $10^{-4}<T_{\text {kin }}<15 \times$ $10^{-3} \mathrm{~J}$ [74]. Abate and Durian [96] track particles in an air vibrated monolayer of spheres. They obtain $T_{\text {kin }}=10^{-6} \mathrm{~J}$.

These results are summarised in table 5.3. The most obvious observation from the table is that the kinetic granular temperatures are spread over three decades. This is because very different driving has been used in the experiments. Our estimates of $T_{\text {kin }}$ and $\langle\vec{v}\rangle$ are within the range of the literature results.

\begin{tabular}{|c|c|c|c|}
\hline Author & Method & $\langle\vec{v}\rangle(\mathrm{mm} / \mathrm{s})$ & $T_{\text {kin }}(\mathrm{J})$ \\
\hline Abate \& Durian [96] & particle tracking & $\approx 3$ & $10^{-6}$ \\
Losert et. al [74] & particle tracking & $30-120$ & $10^{-4}<T_{\text {kin }}<15 \times 10^{-3}$ \\
Menon \& Durian [66] & DWS & 1 & $10^{-6}$ \\
\hline Us & DWS & 6 & $3.4 \times 10^{-5}$ \\
\hline
\end{tabular}

Table 5.3.: Kinetic granular temperatures and mean velocities in comparison. 


\subsection{Conclusions}

In this chapter, we measured and calculated the transport mean free path, $l^{*}$, with a view to using this quantity to measure the kinetic granular temperature, $T_{\text {kin }}$.

In section 5.2.1, the cosine-angle-dependent intensity of light transmitted through a granular sample was measured, from which we were able to measure the extrapolation length, $z_{e}$. We saw that we were not able to improve on the theoretical value of $z_{e}$ by looking at the $P(\mu) / \mu$ data. The transmissivity as a function of sample thickness, $T(L)$, was measured and this data fitted with three expressions in order to obtain $l^{*}$. We saw that the weak absorption equation did not describe our data at all well. The strong absorption equation proved too insensitive to $l^{*}$ to be of genuine use. The full solution was an excellent fit to the data. We observed that our experiments are in the regime of $l^{*} \approx L_{a}$, where neither the weak nor the strong absorption approximation holds, which may explain their failure to describe our data. In any case, the full solution for $T(L)$ (equation 5.5 is to be preferred over either simplification. We saw that the full solution fit was not as good at describing our data at small $L$ and $\phi$, indicating that we are pushing the limits of the diffusion approximation in this range. $l^{*}$ and $L_{a}$ were measured for the same sample at three different volume fractions, $\phi$. We saw that $l^{*}$ and $L_{a}$ increase with decreasing $\phi$, exactly as we would expect. The attenuation length $l_{a}$ was about constant for all $\phi$; again, this result is exactly in line with expectation.

In section 5.2.2, we saw that we were unable to resolve the coherent backscattering cone for our granular sample using the set-up as it stands. Although it is possible that some tuning of the spatial filter and increasing the distance between the sample and the camera could increase experimental resolution somewhat, it is unlikely that it will improve things enough for us to measure an $l^{*}$ of, say, 1.3 $\mathrm{mm}$. Furthermore, our data were dominated by strong glory.

We made predictions about the incoherently backscattered spot in section 5.2.3 based on predictions from Mie theory. Our predictions suggested that the technique may well be sensitive enough to resolve the changes in the backscattered spot brought about by changing $\phi$. Our preliminary measurements bore this out: we were able to see a clear shift in the radially averaged intensity of the spot when varying $\phi$. Again, strong glory was visible, which may prove obstructive when using this method to make quantitative measurements.

In section 5.3, we predicted $l^{*}$ for our small Mo-Sci grains using the methods of Mie, Huffman, Snabre, Mätzler [93, 94]. Our predicted $l^{*}$ were of the same order of magnitude as our transmission measurement, but greater. The experimental $l^{*}$ is smaller than the calculated values because real grains are not perfectly smooth or spherical. We compared all our experimental and theoretical data with literature results, and saw that our work is in good agreement with them. This is true of the magnitude of $l^{*}$ as well as the influence of scattering anisotropy on $l^{*}$. 
Finally, we used our measurement of $l^{*}$, together with a measurement of the speckle decorrelation time $\tau$ (chapter 4 ) to estimate the mean velocity of the grains, $\langle\vec{v}\rangle$, and their kinetic granular temperature, $T_{\text {kin. }}$. We found $\langle\vec{v}\rangle$ in good agreement with visual inspection of the granular bed in motion. We compared our estimates with the literature; both $\langle\vec{v}\rangle$ and $T_{\text {kin }}$ are within the range of the literature results. 



\section{Part III.}

Outlook and Summary 



\section{Outlook}

In this chapter, we present four promising avenues of research that have presented themselves during the course of our work. We offer more than speculation and suggestion; we present only ideas we can support by proof-of-concept experiments or calculations. Two of the topics are introduced in direct response to puzzling aspects of the foregoing research, the other two are larger in scope and could each justify a thesis of their own, should they be pursued further.

In the section 6.1, we address the question of the unexpected roughening behaviour encountered in section 2.3. In section 6.2 the role of grain roughness in wet granular media is discussed. In section 6.3 we place our concept of kinetic granular temperature in the context of other granular temperatures and introduce a method for measuring the fluctuation-dissipation granular temperature, $T_{\mathrm{f}-\mathrm{d}}$. In the last and most extensive section of the outlook (section 6.4), a novel granular material is presented which we see as having great potential in the study of force networks in granular media. Furthermore, it suggests itself as an intermediate system to bridge the gap between granular and emulsion physics.

\subsection{Roughening and inhomogeneity}

In chapter 2, we etched glass grains in ammonium bifluoride and observed two types of roughening behaviour: a monotonic increase in feature width and depth with etch time for the small Mo-Sci grains, and a broadening behaviour for the two larger types of grains, whereby surface features became wider but not deeper as a function of etch time. What mechanism could lead to this second type of feature growth is a question that falls outside the scope of the current work, but one could speculate that the stresses built up in the glass grains during their solidification are almost certainly anisotropic, and that this could lead to different sensibility to the etching chemicals in the radial and lateral directions. One might also expect that grains with a higher curvature offer a greater range of angles of attack to the etching agents, although this ought only to be a small effect for spheres as large as ours. In either case, one would expect a different type of etching to that on, say, float glass, and this is indeed what we see: figure 6.1 shows a soda-lime microscope slide and a Mo-Sci glass sphere both etched for four minutes in $\mathrm{NH}_{4} \mathrm{FHF}$. The microscope slide shows regular, angular holes approximately $1.6 \mu \mathrm{m}$ in diameter etched in an otherwise smooth surface; the glass sphere is more homogeneously etched. Yet the only obvious differences 
between the microscope slide and the glass grains are in the quality of the sodalime glass (Mo-Sci spheres are made of recycled car windows) and in the way the objects were made: the slide by floating, the spheres presumably by spraying.

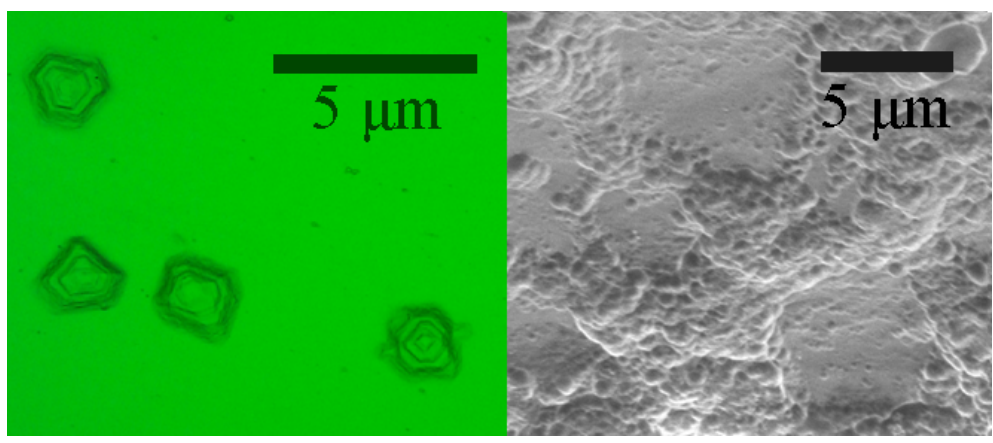

Figure 6.1.: Left: a microscope slide etched in $\mathrm{NH}_{4} \mathrm{FHF}$ for four minutes. It shows a different type of roughness to the glass sphere (right) that has been subject to the same treatment.

\subsection{Wetting and roughness}

\section{Background and motivation}

Granular materials gain a new dimension when a small amount of fluid is also present. The roughness of the grain surfaces plays an important role in determining the physics of wet granular matter [43]. Fluid generates adhesion between grains by means of capillary bridges that form at the contact points between grains. The mechanism is especially strong for hydrophilic grains such as glass or sand. Scheel et al. [97] showed that only a few volume percent of water is enough to stabilise granular packings against compressive and shear forces. Anyone who has ever built a sand-castle will have observed this phenomenon.

There is also a physics of "humid granular media" 98] that takes place at fluid volumes below that necessary to build capillary bridges, and in the presence of surface roughness. This was explored theoretically by Halsey and Levine in 1998 [43. They assume that the fluid accumulates at the particle contacts (which was later ascertained by Mason et al. [99]) and they identify three adhesive regimes depending on the volume of liquid at the contact points and the roughness of the grain surface. This is illustrated in figure 6.2(a).

According to Halsey and Levine [43], the Hurst exponent, $H$, and three length scales play a role in determining the adhesion between moist grains: $R, \xi_{\text {vert }}$ and $\xi_{\text {lat }}$ (figure 6.2(b)). The radius of curvature of the meniscus, $r$, is assumed to be approximately the distance between the two grains at contact, which is assumed to be the typical height of the asperities, $r \approx \xi_{\text {vert }}$. 
(a)

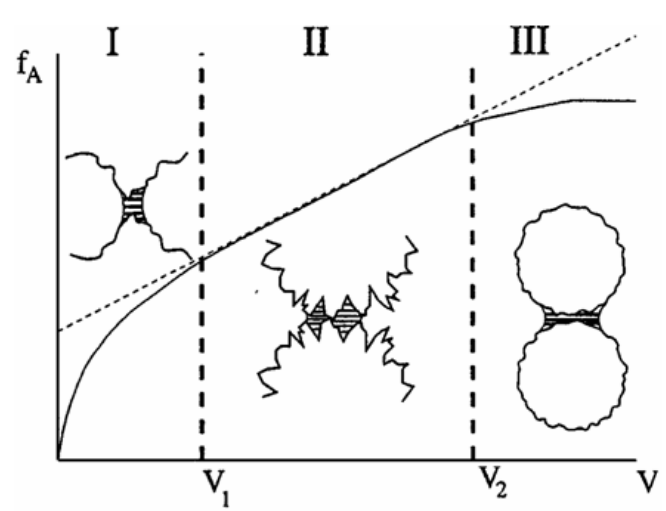

(b)

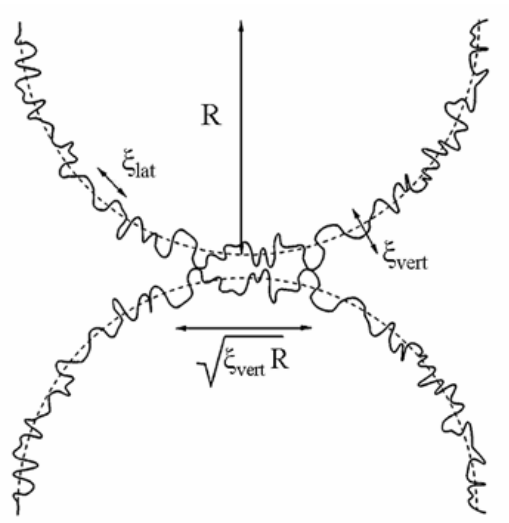

Figure 6.2.: (a) Three regimes of adhesion force, $f_{A}$, between rough spheres. As the volume of the wetting layer, $V$, increases, the contact goes from the asperity regime (I), through the roughness regime (II) to the spherical regime (III). Figure taken from [43]. (b) The contact between two rough spheres of radius $R$. $\sqrt{\xi_{\text {vert }} R}$ is the length of the contact zone. Figure taken from [43, our labels.

\section{Calculations}

Could we observe the three wetting regimes suggested in reference [43] in our etched grains?

Figure 6.3 shows our calculation of the adhesive force, $f_{A}$, as a function of wetting volume, $V$ for the small Mo-Sci grains. We assume a granular packing in air with water as the wetting fluid. The figure shows that it should be possible to measure $f_{A}$ as a function of etch time for fixed $V$, and possibly to measure $f_{A}(V)$ for the roughest grains. The figure suggests that we are unlikely to observe wetting regimes I and II for unetched grains.

The apparatus for measuring $f_{A}(V)$ could be a split-bottom plate upon which a "sandcastle" of the wetted grains has been built. The two parts of the plate could be pulled apart to measure the tensile strength of the pile. The force at an individual contact could be measured using an atomic force microscope by gluing one grain to the tip of the cantilever and holding the other grain fixed.

\subsection{Other granular temperatures}

As was mentioned in chapter 1 , $T_{\text {kin }}$ is not the only definition of granular temperature. A second definition is the fluctuation-dissipation granular temperature, $T_{\mathrm{f}-\mathrm{d}}$, defined in analogy to Einstein's fluctuation-dissipation theorem of 1905, which says that 100$]$ 


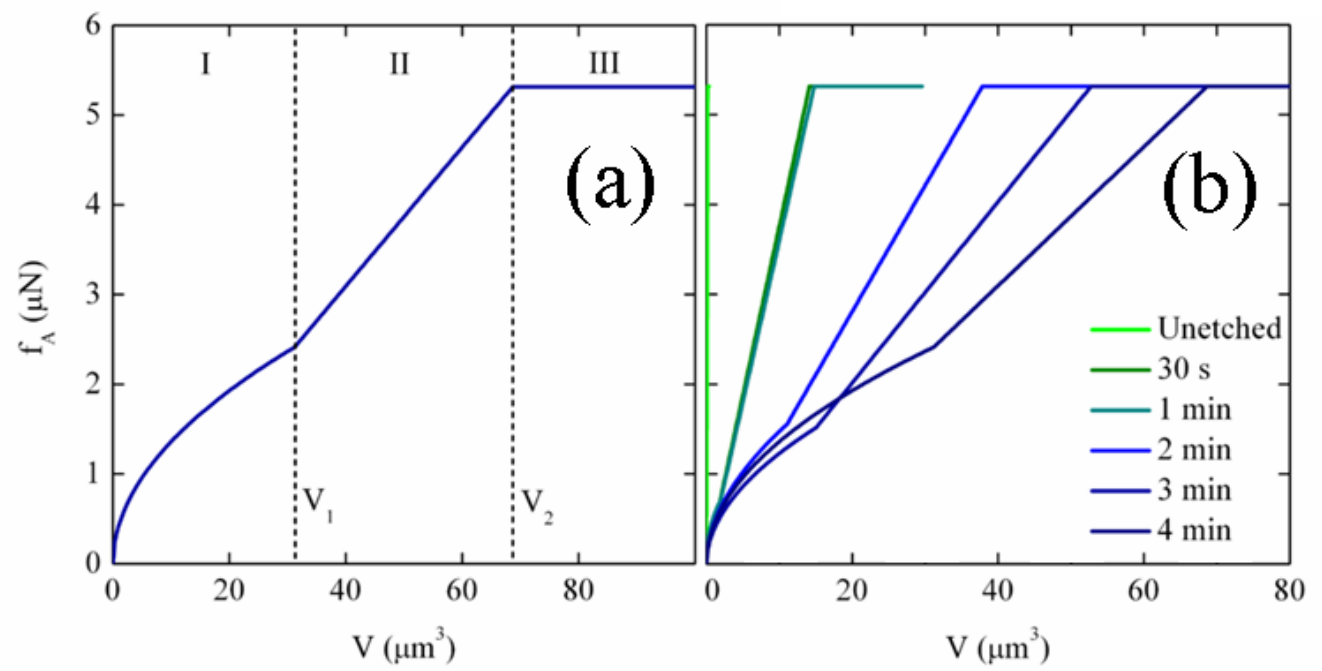

Figure 6.3.: (a) Calculation of the adhesive force as a function of wetting volume for the small Mo-Sci grains etched for four minutes. As $V$, increases, the adhesive force at the contact goes from the asperity regime (I), through the roughness regime (II) to the spherical regime (III). (b) is the same calculation for the small Mo-Sci grains for all $\mathrm{NH}_{4} \mathrm{FHF}$ etch times.

$$
D=\mu k_{B} T \text {. }
$$

Here, $D$ is the diffusion constant of the molecules in a fluid and $\mu$ is the mobility of a particle suspended in this fluid. Song et al. measured the granular version by looking at the mobility and diffusivity of tracer particles in a Couette cell [101]. D'Anna et al. used a torsion pendulum to measure $T_{\mathrm{f}-\mathrm{d}}[102]$ in a shaken granular sample, and Buck [103] also used a torsion pendulum to measure $T_{\mathrm{f}-\mathrm{d}}$, but for in water-fluidised bed. Figure 6.4 shows a set-up we built to measure $T_{\mathrm{f}-\mathrm{d}}$, based on the work of Buck [103] and using some of his equipment. The fluctuations of the granular medium are measured by letting the pendulum rotate freely in the sample, driven by collisions with the grains. The dissipation can be measured by oscillatory driving of the pendulum via the magnet coils.

A third definition of granular temperature comes from Edwards and Oakeshott [17], who considered the way in which a granular material explores configuration space. In this formalism, a compactivity variable, $X=\frac{\partial V}{\partial S}$ is defined as the granular analogue to the temperature $T=\frac{\partial E}{\partial S}$ in equilibrium thermodynamics, where $V$ is the volume, $S$ the entropy and $E$ the energy [104].

In equilibrium thermodynamics, the kinetic, fluctuation-dissipation and conformational temperatures are of equal magnitude. But there is no reason to assume that this is the case for their granular equivalents, $T_{\text {kin }}, T_{\mathrm{f}-\mathrm{d}}$ and $X$, defined for 


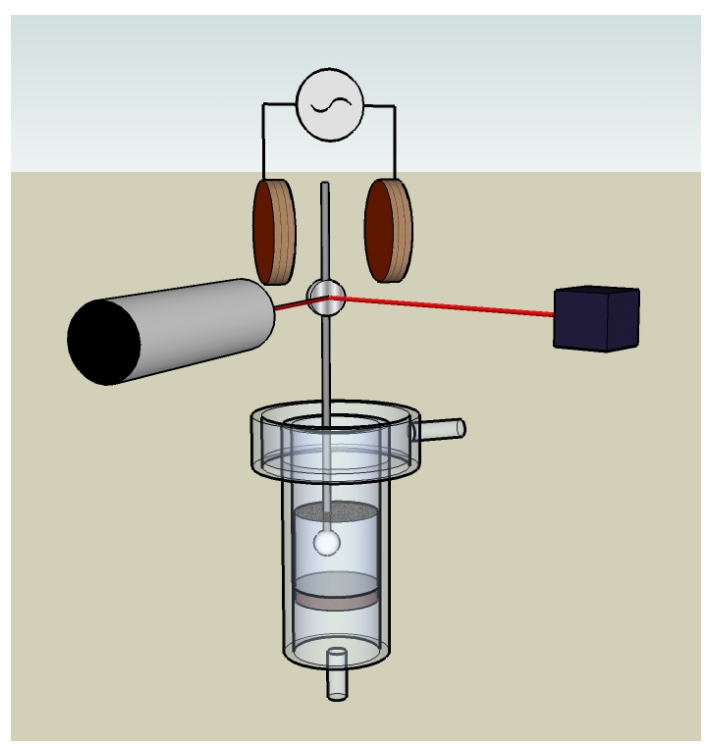

Figure 6.4.: Sketch of the torsion granular thermometer we have built. The torsion pendulum is immersed in a fluidised granular bed (the sample). A laser pointer is reflected by a mirror mounted on the pendulum shaft onto a four-quadrant diode, which measures the pendulum displacement. The pendulum is driven in one of two modes: "fluctuation" (by grain collisions) or "dissipation" (driven by the magnet coils).

a driven granular system which is far from equilibrium. Ultimately, it is desirable to be able to compare all three granular temperatures for one and the same granular system 1 A water-fluidised bed could be the right system in which to make this comparison.

\footnotetext{
${ }^{1}$ This comparison was the original aim of the research presented in this thesis, but it was not possible to realise it in the time available.
} 


\subsection{An ideal granular material}

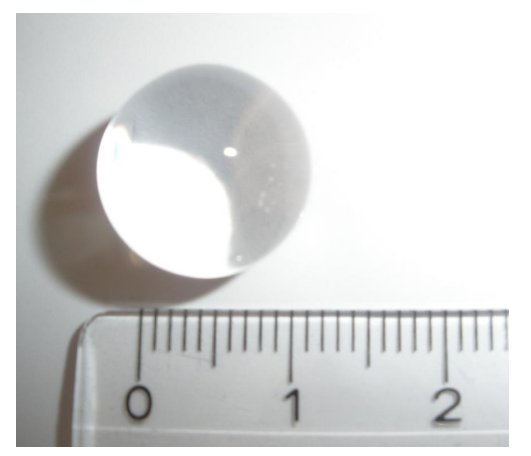

Figure 6.5.: An Aqualino sphere after 24 hours in desalinated water.

A very promising granular material can be found in the form of Aqualinos. These are commercially available spheres of a co-polymer of acrylic acid and sodium acrylate and measure approximately $1 \mathrm{~mm}$ diameter when dry. When desalinated water is added

- they swell to about ten times the diameter (1,000 x the volume); see figure 6.5 .

- they are perfectly spherical, perfectly smooth and virtually frictionless;

- they are neutrally buoyant in water (so gravity can be "switched off");

- they have the same refractive index as water (figure 6.6);

- they can be made to shrink slightly by adding salt, then flushed to re-swell;

- they are slightly polydisperse $(8 \mathrm{~mm}<$ diameter $<13 \mathrm{~mm})$, but can easily be sorted;

- they are elastically soft: they can be easily deformed by hand.

These properties open several avenues for scientific exploration. Their optical properties allow the three-dimensional structure of packings to be readily measured. Their mechanical softness provides a probe for the forces existing inside a packing. Their neutral buoyancy in water makes them insensitive to gravity; they produce weightless, isotropic packings. Their low coefficient of friction ${ }^{2}$ allows the formation of high-density packings.

\footnotetext{
${ }^{2}$ The co-efficient of friction of hydrogels on polystyrene surfaces is of the order of $10^{-4}$ [105],
} about 100 times lower than that of Teflon. 

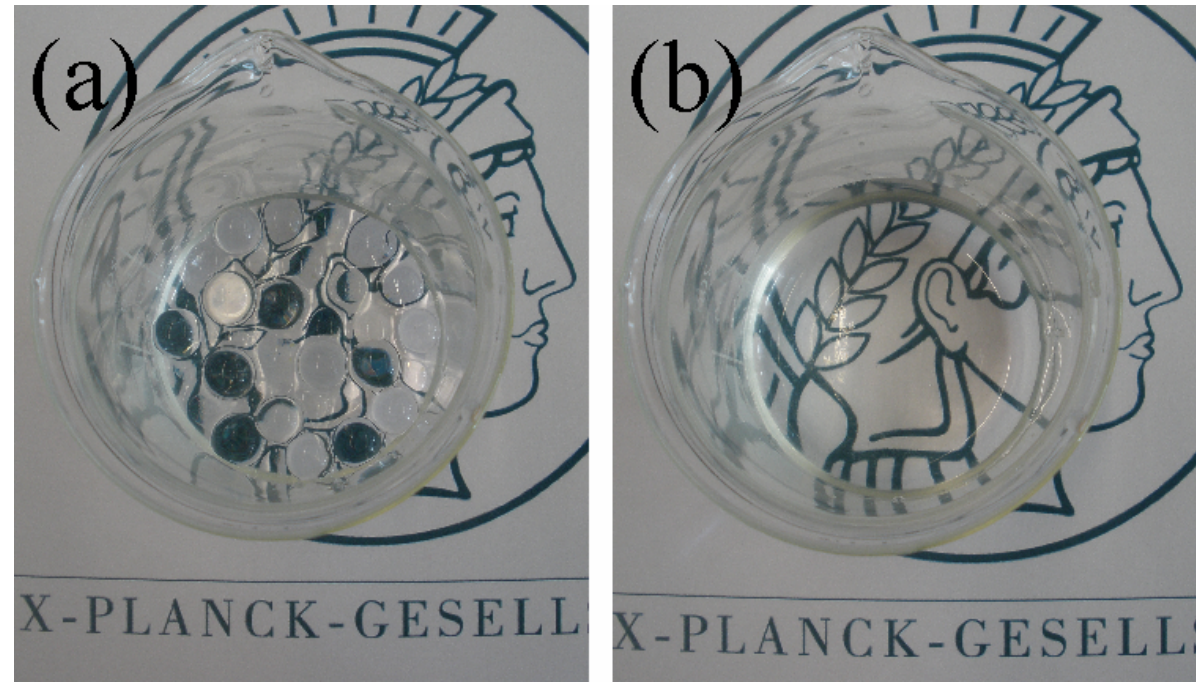

Figure 6.6.: polymer spheres (a) without; and (b) with water. The almost perfect index matching makes them invisible in water.

\subsubsection{Polymer spheres and the physics of packings}

The usual method of visualising index-matched objects in three dimensions is to place them in a rectangular container, add a fluorescent dye to the surrounding liquid and shine a laser sheet through it. The container is then moved such that the plane of the laser sheet scans through the entire depth of the sample. The camera captures a stack of images in which the objects are dark and the water between them bright. This is illustrated in figure 6.7.

A proof-of-concept experiment under a Leica confocal microscope indicated that this method should indeed work for the Aqualinos (figure 6.8). A second test of the type shown in figure 6.7 using a laser sheet in a sample $10 \mathrm{~cm}$ in thickness (approximately ten sphere diameters) showed no degradation in the quality of the image even at full depth.

Mukhopadhyay and Peixinho [105] tested a similar system by creating polymer spheres out of polyacrylamide hydrogel and letting them swell in rhodamine solution (figure 6.9). They measured the packing fraction, $\phi$, as a function of the normal force applied to the packing; and the coordination number and pair distribution function, $g(r)$ as a function of $\phi$. However, their error bars showed no improvement on X-ray tomographic data taken on glass spheres [106], which is well to be expected for the small number of particles they used $(N=600)$. We speculate that an experiment with $5,000<N<10,000$, such as was used in the $\mathrm{X}$-ray data of reference [106], may well offer an improvement.

It would be nice to use these grains to reproduce the classic numerics experiment of swelling beads in confinement. Since they shrink when a little salt is added, they could be packed and constrained in a saline solution which is then 


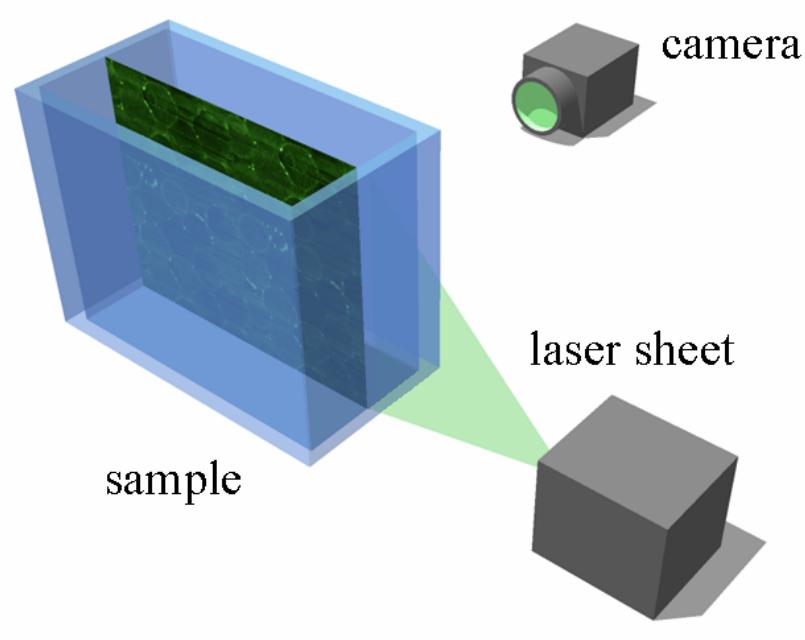

Figure 6.7.: The index-matched sample with fluorescent dye is moved through a laser sheet. The camera captures a stack of images which can be used to construct the three-dimensional arrangement of the polymer spheres.

flushed with distilled water to make them re-swell.

\subsubsection{Interparticle forces}

The grains are soft and deform nicely under a little pressure: two fingers are enough to compress them to a half of their diameter. This suggests that the force acting on a sphere can be measured directly from its deviation from the spherical, and that the force at a contact between two spheres can be measured from the size of the contact area between them. A simple calibration measurement of the Aqualinos showed that they obey Hooke's law for strains of up to 0.1 with a stiffness modulus of $14 \mathrm{~N} / \mathrm{mm}$ : this is shown in figure 6.10 .

A quasi-2D proof-of-concept experiment was carried out to test if force networks could be measured this way. A glass tank measuring $10 \mathrm{~cm}$ in length, $5 \mathrm{~cm}$ in depth and $20 \mathrm{~cm}$ in height was filled with $\sim 500$ polymer spheres and millipore water coloured with fluorescein dye. A plate that fitted loosely inside the cell was placed on top of the spheres; the plate could be pressed down to compress the sample. A laser sheet $(\lambda=532 \mathrm{~nm})$ was shone through the sample at a depth 2.5 $\mathrm{cm}$ from the front of the tank. A single slice image was taken using a black-andwhite 8-bit CCD. The image was analysed as if it were $2 \mathrm{D}$ data in the following hand-waving and subjective fashion: where image quality permitted, the lengths of the inter-particle contact lines were measured. Coloured lines were then drawn between the grain centres; the length, thickness and colour of the line encoding the length of the contact line, which was taken as an indicator of the magnitude of the inter-particle forces. Three realisations of the experiment were analysed: 


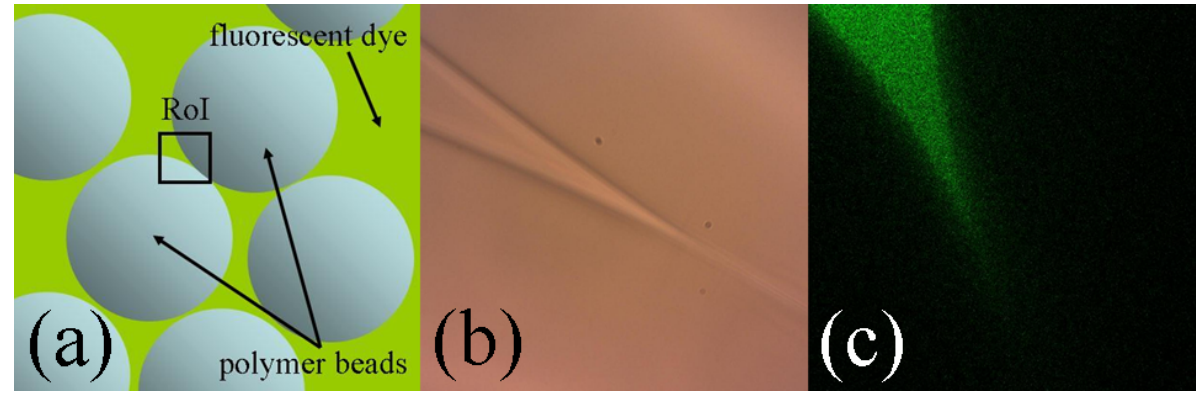

Figure 6.8.: The index-matched sample with fluorescent dye is examined with a $10 \mathrm{X}$ objective on a Leica confocal microscope. Fluorescein dye was used with $\lambda_{\text {excitation }}=496 \mathrm{~nm}$ and $\lambda_{\text {emission }}=510-630 \mathrm{~nm}$. (a) Sketch of the sample showing the region of interest (ROI) in the micrographs; (b) white light micrograph of the contact point between two spheres; (c) fluorescent image of the same.

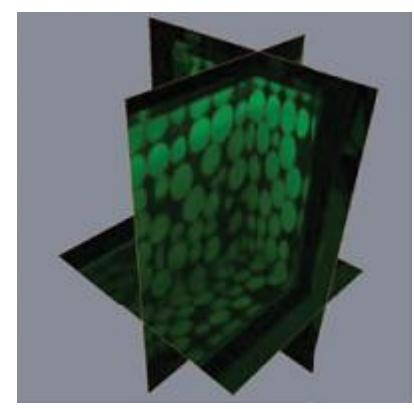

Figure 6.9.: Mukhopadhyay and Peixino visualise a packing of fluorescent polyacrylamide spheres in water. Figure taken from [105].

(a) a slightly compressed (strain $\sim 0.15$ ) random packing; (b) a more strongly compressed packing that had undergone rapid compression and decompression and had therefore formed lanes (strain $\sim 0.2$ ); and (c) packing (b) after increasing the strain to about 0.3 , which caused a spontaneous global re-arrangement of the spheres to a hexagonal packing. The result is shown in figure 6.11. The figure shows that the method has great potential.

\subsubsection{An intermediate system}

As Mukhopadhyay and Peixinho noted in reference [105], the Aqualinos mediate between granulates and emulsions. They have very low friction and are easily deformable, as are the bubbles in an emulsion. But they are elastically soft (rather than having a softness controlled by surface tension), do not coarsen over time, and there is no potential gradient between them: all features of a granular system. 


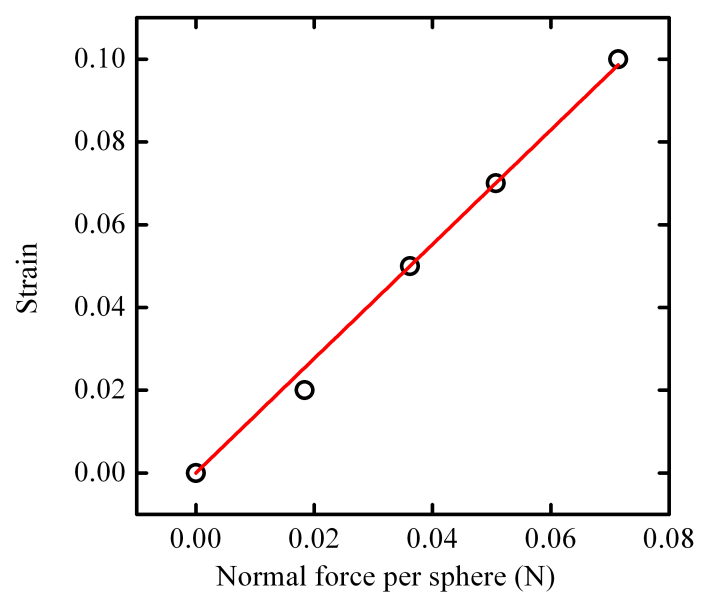

Figure 6.10.: A quick and dirty calibration of the force-strain behaviour of Aqualinos. The red line is a linear fit to the data. 


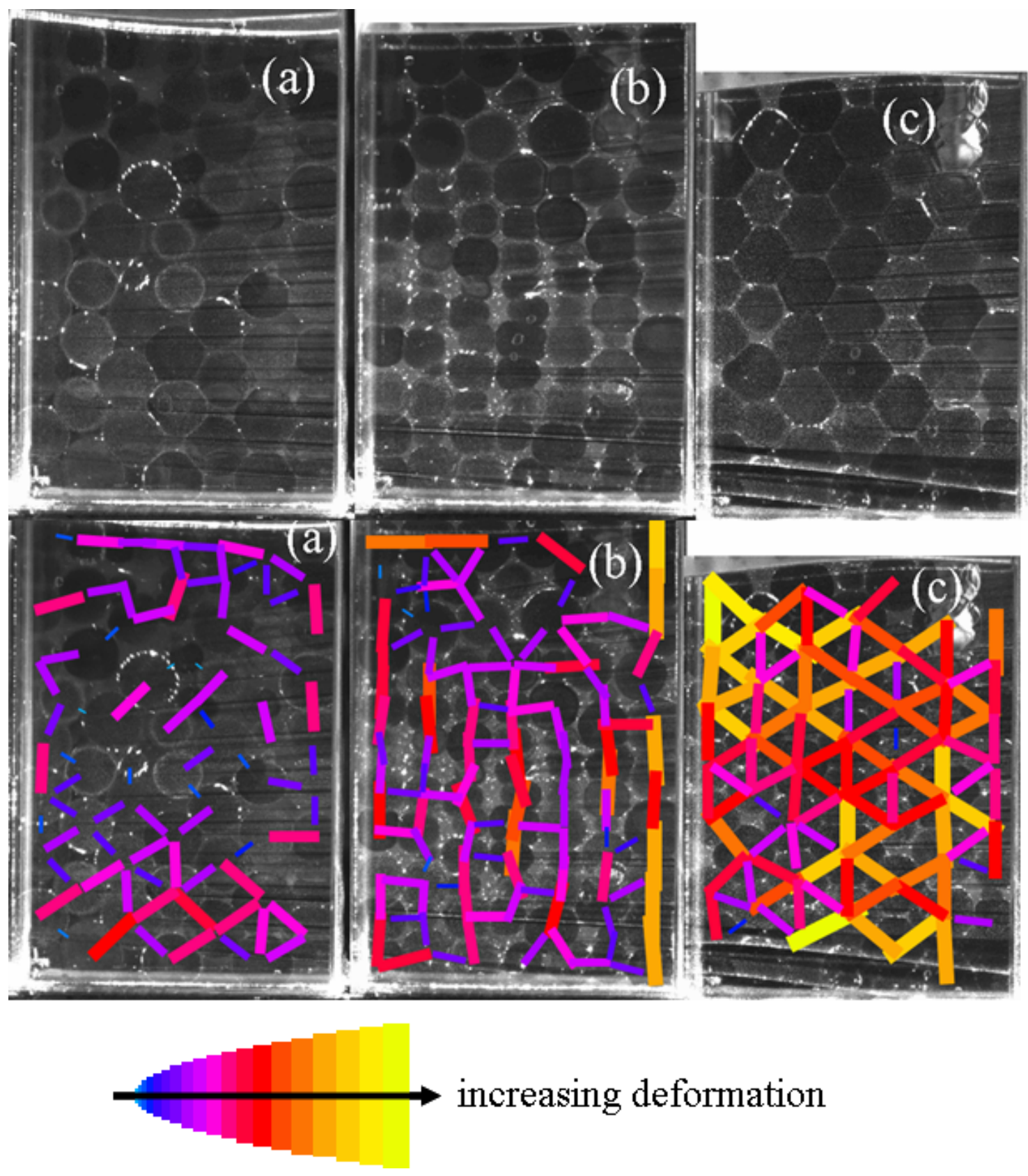

Figure 6.11.: Measuring force networks with Aqualino packings. (a) A slightly compressed packing. (b) A compressed packing that had a square symmetry due to lane formation. (c) A strongly compressed packing that had naturally taken on a hexagonal symmetry. The coloured bars in the lower three images encode qualitatively the inter-particle forces. 



\section{Summary}

In this thesis, we presented our studies of friction and light transport in granular media.

First, we looked at friction. We noted the important role played by friction in granular statics (volume fraction, force networks, heap stability) and dynamics (avalanches, flow through openings, granular cooling). Our aim in part II was to modify the surface roughness of glass spheres as a means of tuning inter-particle friction. We succeeded in achieving this aim, as we showed in chapters 2 and 3 .

In chapter 2, we presented two chemical etching procedures to modify the roughness of glass spheres: one to smooth them and one to roughen them. We produced 21 batches of grains that differed in size, manufacturer, etch time and etch protocol. The roughness of the grain surfaces was measured by white light interferometry (WLI). A method of statistical roughness analysis was developed to measure roughness on a spherical surface, taking account of the angle of sight of the WLI measurement. This analysis gave us three robust, quantitative measures for roughness: a vertical and a lateral roughness length scale $\left(\xi_{\text {vert }}\right.$ and $\left.\xi_{\text {lat }}\right)$ and the Hurst exponent, $H$. The roughening protocol was very successful: $\xi_{\text {lat }}$ and $H$ changed monotonically with etch time and $\xi_{\text {vert }}$ increased as a step function. By these measures, we affirmed that we are able to tune the roughness of soda-lime glass spheres, albeit in not in a straightforward way. The smoothing protocol did not bring about a resolvable change in the roughness of the grains. Inspired by the Mentos and Coke experiment, a promising bulk method for measuring grain roughness was suggested; here, the nucleation of bubbles in a granular sample immersed in cola was shown to be an indicator of grain roughness.

Having modified the roughness of our grains, we then measured the effect that grain roughness has on inter-particle friction.

In chapter 3, the underwater angle of repose, $\alpha_{r}$, was investigated as a measure of inter-particle friction. We confirmed that we are indeed able to tune friction, though here, too, the relationship between $\alpha_{r}$ and etch time is not a straightforward one for all grain types. The measurements of $\alpha_{r}$ were verified by measuring the angle of internal friction in stationary flow, $\alpha_{s f}$, using a ring shear tester. We then looked at other variables that might be expected to depend on friction. We were able to confirm our two hypotheses: (1) that the volume fraction, $\phi$, decreases with increasing friction; and (2) that piles with a greater $\phi$ are more mechanically stable.

In part II of the thesis, we looked at light transport in granular media. We noted that diffusive light transport can be used to give us two useful pieces of 
information about the dynamics inside a 3D granular sample: a length scale, $l^{*}$, and a time scale, $\tau$. These two quantities allow us to calculate the kinetic granular temperature, $T_{\text {kin }}$.

In chapter 4, we looked at ways of driving granular media and showed how a water-fluidised bed can be used to drive grains homogeneously and precisely over a range of kinetic energy and volume fraction. We successfully demonstrated that diffusing wave spectroscopy (DWS) can be used to measure the time scale of granular motion, $\tau$. We observed that $\tau$ is shorter for higher flow rates, exactly as would be expected.

In chapter 5, we measured and calculated $l^{*}$, the transport mean free path with a view to using this quantity to calculate $T_{\text {kin }}$ from $\tau$. We used three different experimental methods. In the first method, the transmissivity as a function of sample thickness, $T(L)$, was measured and the data fitted in order to obtain $l^{*}$. Our data were well described by diffusive light transport theory. $l^{*}$ was measured for the same granular sample at three different volume fractions, $\phi$. We saw that $l^{*}$ increases with decreasing $\phi$, exactly as we would expect. We were unable measure $l^{*}$ by our second experimental method, coherent backscattering, because our experimental resolution was too poor. Furthermore, our data were dominated by glory. Our preliminary measurements using a third method, incoherent backscattering, showed the technique to have good potential: we were able to see a clear shift in the radially averaged intensity of the backscattered spot when varying $\phi$.

We compared our transmission measurements of $l^{*}$ with theoretical calculations and literature results, and found good agreement. This was true of both the magnitude of $l^{*}$ as well as the influence of scattering anisotropy on $l^{*}$. Finally, we used our measurement of $l^{*}$, together with a measurement of the speckle decorrelation time, $\tau$, to estimate the mean velocity of the grains, $\langle\vec{v}\rangle$, and their kinetic granular temperature, $T_{\text {kin }}$. We found $\langle\vec{v}\rangle$ to agree well with visual inspection of the granular bed in motion. Both $\langle\vec{v}\rangle$ and $T_{\text {kin }}$ were found to be in the same range as literature results.

In the outlook, we introduced a novel granular material which we see as having great potential in the study of granular force networks in granular media. Furthermore, it suggests itself as an intermediate system to bridge the gap between granular and emulsion physics. 


\section{Appendix}

\section{A. Side walls and flowing granular media}

In section 3.1, the angle of repose, $\alpha_{r}$, was measured in a cell of width $B=40$ grain diameters. The purpose of appendix $\mathrm{A}$ is to demonstrate that the width of the cell is sufficient to justify our choice of $B$.

\section{Model}

This continuum model is based loosely on the method of references [58] and [107]. Consider a granular pile of width $B$ that makes the angle $\alpha$ to the horizontal, sketched in figure A1. Since grain motion is at the surface of the pile, we consider a slab $B \times L \times H$ of those grains, where $H$ is very small (a few grain diameters). It has the mass $\rho B L H$, where $\rho$ is the mean density of the granular material. Lengths are in units of particle diameter.

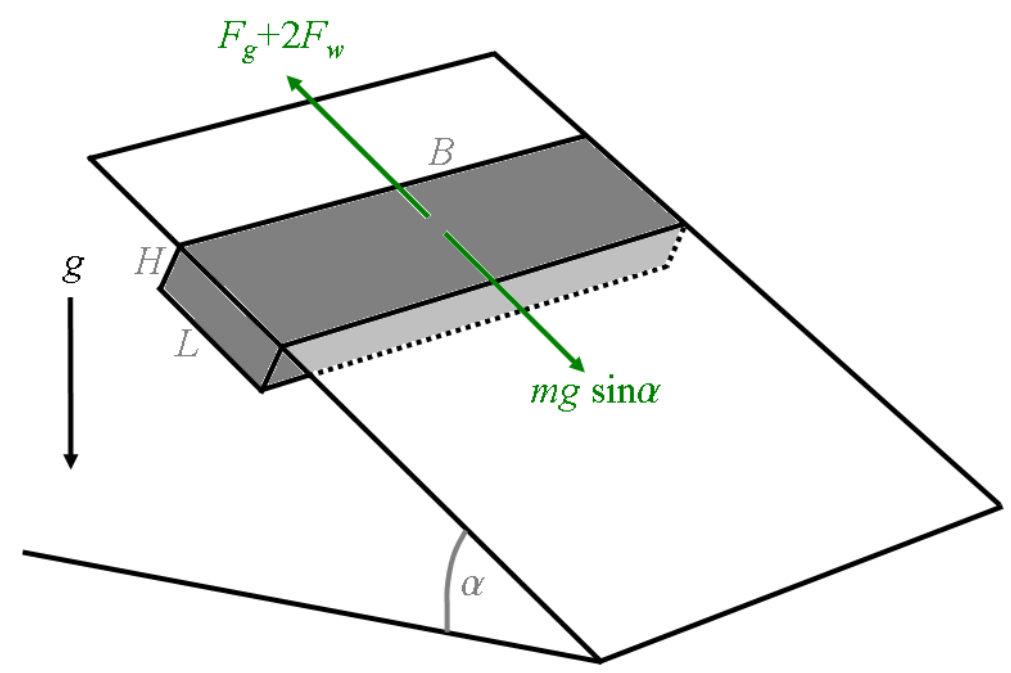

Figure A1.: A granular pile of width $B$ whose surface makes the angle $\alpha$ to the horizontal. We consider the forces acting on a slab (shaded) of width $B$, length $L$ and depth $H$. At force balance, $\alpha=\alpha_{r}$ and the shear forces $m g \sin \alpha$ and $F_{g}+2 F_{w}$ are equal.

The normal force exerted on the base of the slab is given by 


$$
N_{g}=\rho B L H g \cos \alpha,
$$

Where $g$ is the acceleration due to gravity and $\mu_{g}$ is the inter-particle friction coefficient. The subscript $g$ stands for grains. The shear force at the base of the slab is thus

$$
F_{g}=\mu_{g} N_{g}=\mu_{g} \rho B L H g \cos \alpha .
$$

The shear stress on one of the walls is given by

$$
F_{w}=\mu_{w} N_{w}=\mu_{w} L \int_{0}^{H} p(h) \mathrm{d} h
$$

where $N_{w}$ is the normal force on the wall and $\mu_{w}$ is the friction between the grains and the wall. The subscript $w$ stands for wall. $p(h)$ is the pressure as a function of depth, which, if we assume a hydrostatic pressure gradient given by $p(h)=\rho g h \cos \alpha$, gives us the expression for the frictional force between the slab and one wall:

$$
F_{w}=\mu_{w} N_{w}=\mu_{w} \rho L H g \cos \alpha .
$$

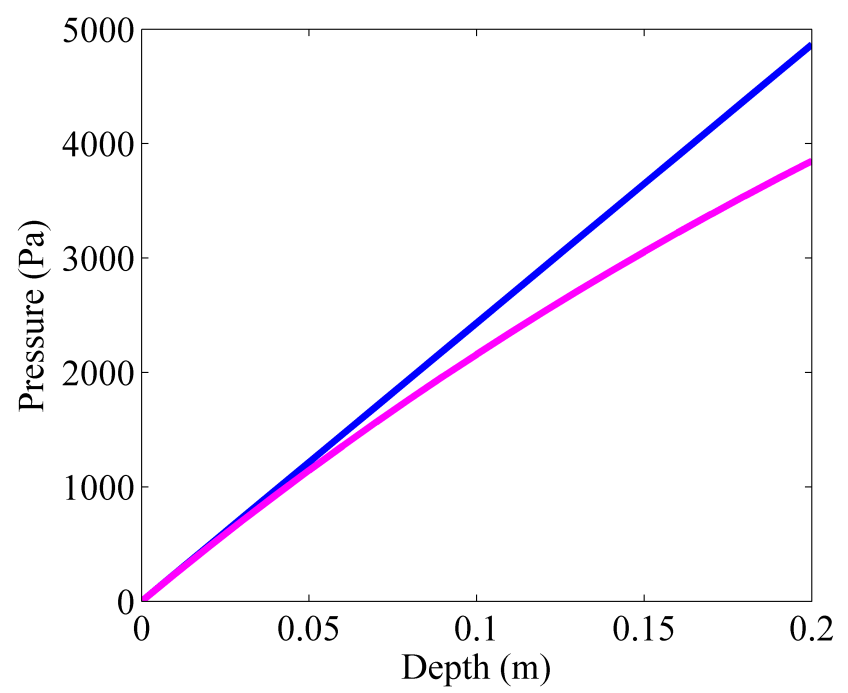

Figure A2.: Hydrostatic (blue) and Janssen (pink) pressure as a function of depth, calculated for a cylindrical vessel of diameter $D=10 \mathrm{~cm}$. The Janssen pressure is given by $\rho g \frac{D}{4 K \mu_{w}}\left(1-\exp \left(-\frac{4 K \mu_{w} h}{D}\right)\right)$; we take $\rho=2480 \mathrm{~kg} / \mathrm{m}^{3}$ as the density of the material, $K=0.2$ as the ratio of horizontal to vertical normal stresses and $\mu_{s}=\tan \left(17^{\circ}\right)$ as the friction between grains and the wall. For a slab of grains of small depth, $H$, it is not important which pressure rule we choose. For simplicity, we take the hydrostatic pressure. Courrech du Pont et al. use the Janssen pressure. 
We use hydrostatic pressure instead of the Janssen law [5] because $H$ is small. Figure A2 shows that the Janssen and hydrostatic pressures are similar for small depths. At force balance (when the slab is just about to slide), $\alpha=\alpha_{r}$ and the tangential forces up and down the slope are equal:

$$
\rho B L H g \cos \alpha_{r}=F_{g}+2 F_{w} .
$$

Dividing by $\rho(H)=\rho g H \cos \alpha_{r}$ and solving for $\alpha_{r}$ gives us

$$
\tan \alpha_{r}=\frac{B_{r}}{B}+\mu_{g}
$$

where $B_{r} \equiv \mu_{w} H$ is a characteristic length describing the influence of the walls on the stability of the pile. When $B_{r} \rightarrow 0$, we recover the expression for Coulomb friction without walls, $\mu_{g}=\tan \alpha_{r}$. The value of $B_{r}$ has to be found experimentally by measuring $\alpha_{r}(B)$; Courrech du Pont et al. obtained $B_{r}=1.3 d$ for $d=3 \mathrm{~mm}$ glass spheres in water; Metayer et al. obtained $B_{r}=0.72 d$ for $d=0.5 \mathrm{~mm}$ glass spheres in air $\left(\left[58\right.\right.$, , 107]. If we take $B_{r}=1 d$, for our cell width $B=40 d$ we make a systematic error of $1.4^{\circ}(6 \%)$ in measuring $\alpha_{r}$.

\section{B. Principles of shear testing}

In section 3.2, we used a ring shear tester to measure the angle of internal friction for stationary flow, $\alpha_{s f}$, for a given preparation protocol. Here, we describe how the yield locus of a granular material is measured and show some experimental results for our etched grains.

The yield locus is a collection of points in the $(\sigma, \tau)$ plane, where $\sigma$ is the normal stress and $\tau$ the shear stress. It describes the flow properties of the granular sample that has undergone a specific, repeatable preparation protocol. To explain how this works, it is necessary to make a small excursion into Mohr's stress circles, a means of expressing stresses in the $(\sigma, \tau)$ plane. The explanation given here is based on Pulver und Schüttgüter: Fließeigenschaften und Handhabung by D. Schulze (reference [60]) and Principles of powder mechanics by R. L. Brown and J. C. Richards [67]. We will look first at a simplified method, then show how the same quantities can be measured using a shear tester.

\section{Uniaxial pressure test}

We measure the first point on the yield locus by the unconstrained uniaxial pressure test. This test is illustrated in figure B3. It is essentially the building and subsequent crushing of a sand-castle: the granular sample is laterally confined and then compressed by the consolidation normal stress $\sigma_{1}$. The confining walls are removed and the consolidated sample is compressed until it yields (called incipient flow). Incipient flow occurs at $\sigma_{c}$. Increasing $\sigma_{1}$ leads to a greater 


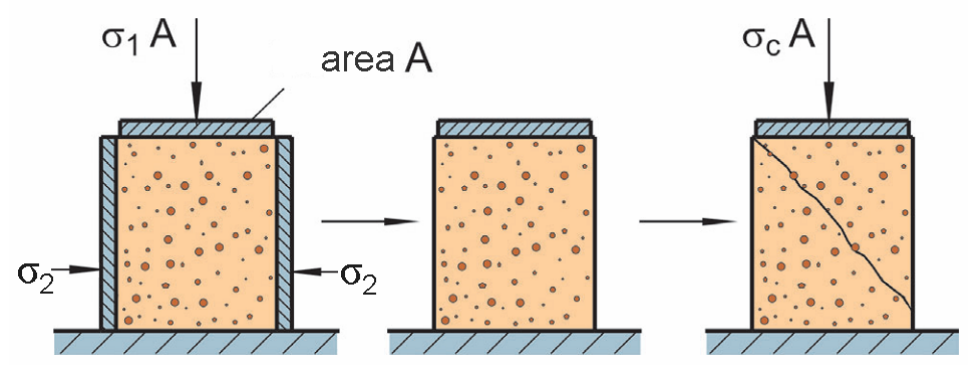

Figure B3.: Uniaxial pressure test. (a) Sample is confined and consolidated with the normal force $N=\sigma_{1} A$, where $A$ is the area. (b) Confining walls are removed. (c) Normal stress is applied until the sample yields (incipient flow). The stress at which this occurs is $\sigma_{c}$. Figure taken from [60.

compression of the sample and to an increase in its strength; for this reason, the yield locus is measured for one specific $\sigma_{1}$. A granular medium is described by a whole family of yield loci, each measured for a different $\sigma_{1}$.

Figure B4 shows how the uniaxial compression test can be represented in the $(\sigma, \tau)$-plane. The Mohr circle stress diagram has normal stress, $\sigma$, on the abscissa and shear stress, $\tau$, on the ordinate. We assume that stresses are uniform in the sample. This is not necessarily justified, but doing so allows us to describe the whole sample in a given state by one Mohr circle. We assume also that friction with the walls is negligible, which allows us to say that the primary stresses in the sample are normal stresses $\left(\sigma_{1}\right.$ and $\sigma_{2}$, figure B3), and if this is the case, the centre of a Mohr circle has to lie on the $\tau=0$ axis.

First, let us look at the consolidation step (figure B3(a)). We apply the consolidating normal stress, $\sigma_{1}$ to the top of the sample. Confinement by the walls leads to a smaller, horizontal stress, $\sigma_{2}$. If we assume friction between the sample and the walls to be negligible, there will be no shear stresses, $\tau$, in the horizontal or the vertical planes of the sample. The stresses in these planes are thus represented in $(\sigma, \tau)$ space by the points $\left(\sigma_{2}, 0\right)$ and $\left(\sigma_{1}, 0\right)$ respectively. These two coordinates, together with the assumption that the centre of the Mohr circle lies on the $\tau=0$ axis, are enough to define the Mohr circle for the consolidation uniquely.

Let us now perform the second part of the uniaxial compression test (figure B3(c)). Since we have no side walls, $\sigma_{2}=0$. This tells us that our Mohr circle goes though $(0,0)$. The normal stress that causes the sample to yield, $\sigma_{c}$, gives us the second crossing point of the Mohr circle with the $\sigma$ axis. This Mohr circle gives us our first piece of information about the yield locus for this particular consolidation: the yield locus is tangent to the Mohr circle for incipient flow.

Clearly, this is not enough information to allow us to plot the yield locus: we need a number of Mohr circles for incipient flow, and they must be for the same consolidation $\sigma_{1}$. To this end, we can repeat the second part of the uniaxial 


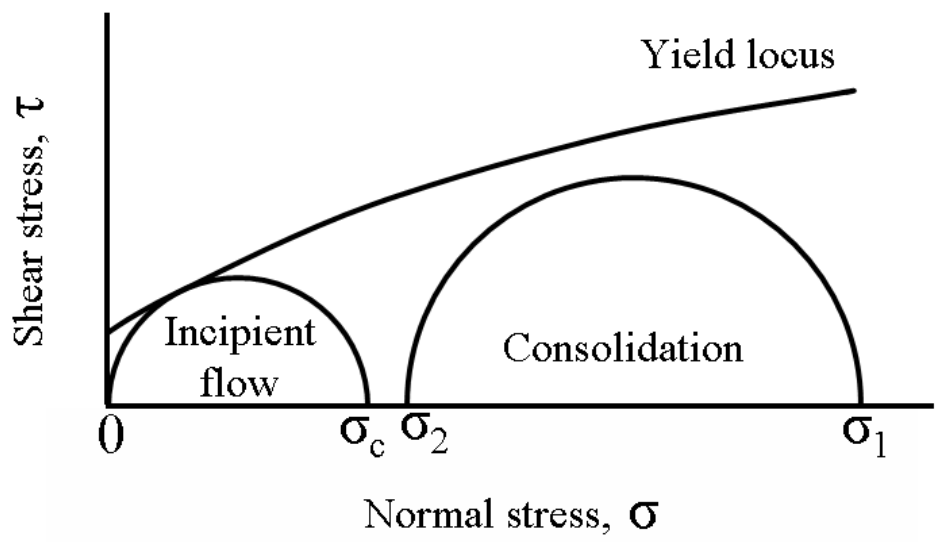

Figure B4.: Mohr circle diagram of the stresses in a granular sample undergoing the uniaxial pressure test. First, it is consolidated (right-hand circle). This corresponds to figure B3(a). Then, a normal stress is applied until the sample yields (incipient flow). The stress at which this occurs is $\sigma_{c}$. This corresponds to figure B3(c). The black curve is the yield locus that we wish to measure. Figure adapted from [60]. Because the diagram is symmetrical about the $\sigma$ axis, only the $\tau \geq 0$ half of the diagram is shown.

pressure test, this time applying a small horizontal confining stress $\sigma_{2}>0$. This gives us the blue Mohr circle in figure B5. We can then repeat the test for a number of $\sigma_{2}$ and thus obtain the yield locus for a given consolidation.

Figure B3(c) shows the sample failing along a clean diagonal yield plane with a well-defined angle to the horizontal, but for non-cohesive grains such as ours, this is not to be taken literally. The fracture plane represents the ratio of normal to shear stresses in a sample at incipient flow, i.e. at the point on the Mohr circle that touches the yield locus. However, this way of visualising things helps us understand how an angle in the $(\sigma, \tau)$ plane can be mapped to an angle such as $\alpha_{s f}$.

\section{Shear test}

In the uniaxial pressure test as it is described above, it is very difficult to produce a homogeneous packing or to see when consolidation is complete. It is also impossible to completely eliminate friction between the sample and the walls, and the technique does not lend itself to serial automised testing. For these reasons, shear testing is used. In shear testing, the consolidation step of the uniaxial pressure test is by means of pre-shearing, and incipient flow is measured by shearing to failure. The shear cell we used is shown in figure 3.7 .

Pre-shear (consolidation) is applied as follows: a normal stress, $\sigma_{\text {pre }}$, is applied to the top of the sample. It is then sheared until steady-state flow sets in. In steady-state flow, the shear stress has a constant value, $\tau_{\text {pre }}$; the sample is said to 


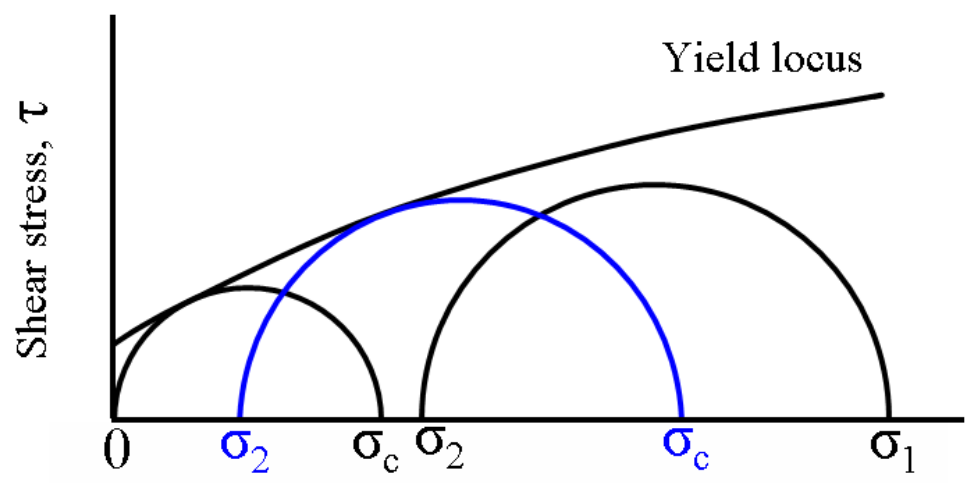

Normal stress, $\sigma$

Figure B5.: A second uniaxial pressure test with $\sigma_{2}>0$ gives us a second circle to which the yield locus is tangent (blue circle and notation). Figure adapted from [60].

be critically consolidated with respect to $\sigma_{\text {pre }}$. A greater $\sigma_{\text {pre }}$ leads to greater $\phi$ and $\tau_{\text {pre }}$ under stationary flow conditions. $\left(\sigma_{\text {pre }}, \tau_{\text {pre }}\right)$ is the pre-shear point (green point in figure B6(b)). It is below the yield locus, just as the consolidation Mohr circle is below the yield radius in figures B4 and B5. After consolidation, the shear direction is reversed until $\tau=0$. The sample is in a well defined, reproducible state. The pre-shear cycle is sketched in the first part of the curve in figure B6(a); an experimental curve can be seen in figure 3.8 .

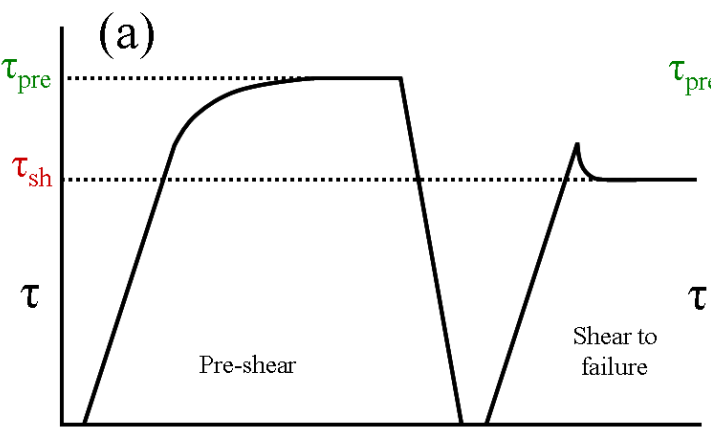

Time (b)

Pre-shear point

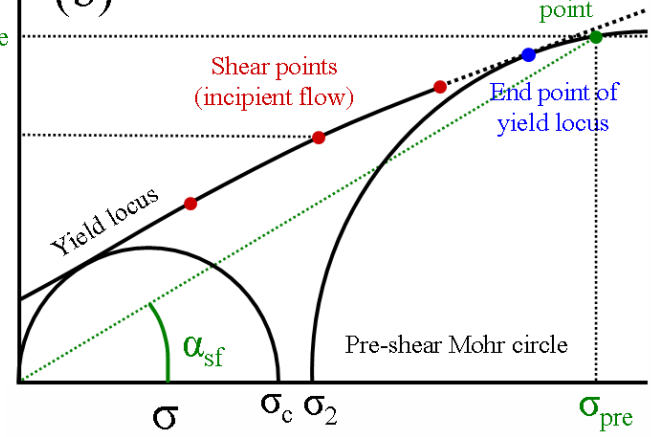

Figure B6.: Measuring the yield locus, unconfined yield strength, $\sigma_{c}$, consolidation stress, $\sigma_{1}$, and angle of internal friction, $\alpha_{s f}$ using a shear tester. (a) shear stress as a function of time for pre-shear (consolidation) and one shear to failure cycle (incipient flow). (b) $\tau(\sigma)$ showing the yield locus and the Mohr circles calculated therewith. $\sigma_{1}$ is the second point (not shown) at which the pre-shear Mohr circle crosses the $\sigma$ axis. Figure adapted from [60.

The second step is shearing to failure (incipient flow), illustrated in the second part of the $\tau$ (time) curve in the same figure. A normal stress $\sigma_{s h}<\sigma_{\text {pre }}$ is 
applied to the sample and again it is sheared until steady-state flow sets in, at $\tau_{s h} .\left(\sigma_{s h}, \tau_{s h}\right)$ is a point on the yield locus. The sample is again pre-sheared at $\left(\sigma_{\text {pre }}, \tau_{\text {pre }}\right)$ to re-consolidate and then sheared to failure for a different $\sigma_{s f}$ (red points in figure B6(b)).

Two Mohr circles can now be constructed to give the unconfined yield strength, $\sigma_{c}$, and the major and minor consolidation stresses, $\sigma_{1}$ and $\sigma_{2}$. The first of these is found by constructing the Mohr circle that goes through $(0,0)$, is tangent to the yield locus and has its centre on the $\sigma$ axis. The second point at which it crosses the $\sigma$ axis is $\sigma_{c}$. The pre-shear Mohr circle is constructed with our knowledge that its centre lies on the $\sigma$ axis, that it is tangent to the extrapolation of the yield locus, and that it goes through the pre-shear point $\left(\sigma_{\text {pre }}, \tau_{\text {pre }}\right) . \sigma_{1}$ and $\sigma_{2}$ are the points at which it crosses the $\sigma$ axis. The angle of internal friction at steady-state flow, $\alpha_{s f}$, is the angle between the $\sigma$ axis and the line joining $(0,0)$ and $\left(\sigma_{\text {pre }}, \tau_{\text {pre }}\right)$. How this analysis looks for a batch of our samples is shown in figure B7. Here, the Mohr circles for determining $\sigma_{c}$ are so small as to be barely visible; this is typical for non-cohesive granular samples.

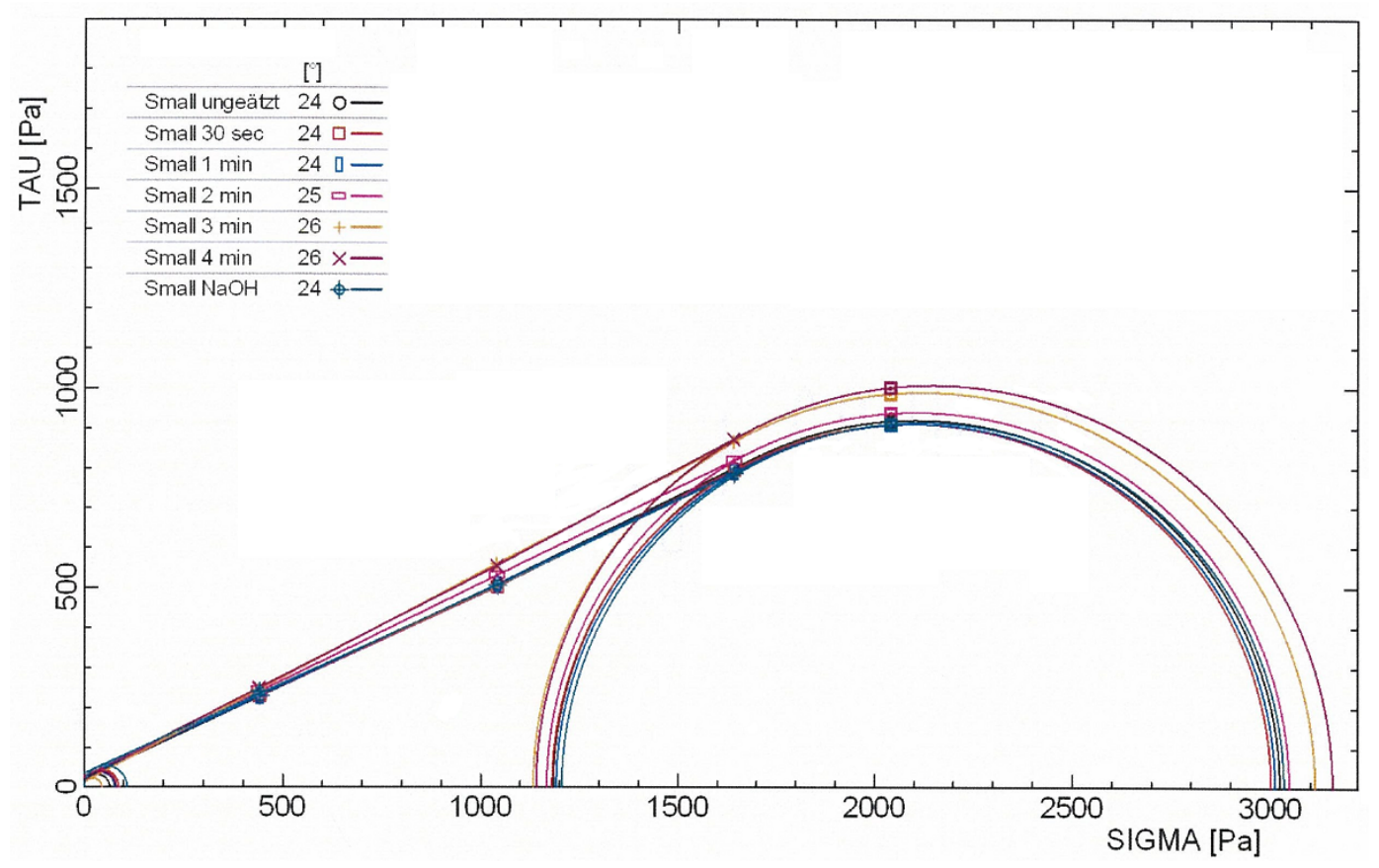

Figure B7.: $\quad \tau(\sigma)$ for all seven small Mo-Sci samples. The diagonal line is the yield locus and the angle between the $\sigma$ axis and the line joining $(0,0)$ and $\left(\sigma_{\text {pre }}, \tau_{\text {pre }}\right)$ (square point) gives the angle of internal friction, $\alpha_{s f}$ (shown in section 3.2). 


\section{Diffusing wave spectroscopy (DWS)}

In section 4.3 , we said that the visibility of a speckle pattern at a given exposure time, $T$, encodes information about the time scale of granular motion, $\tau$. To define visibility quantitatively, one looks at $V(T)$, the normalised variance of the speckle pattern as a function of exposure time. This is defined as

$$
V(T) \equiv \frac{1}{\beta}\left(\frac{\left\langle I^{2}\right\rangle}{\langle I\rangle^{2}}-1\right)
$$

where $1 / \beta \sim 1$ is the number of speckles per pixel, measured without scatterer motion and $I$ is the normalised grey level of a pixel. $\langle\cdots\rangle$ indicates ensemble averaging, not time averaging. $V(T)=1$ for scatterers at rest and $V(T) \rightarrow 0$ when $\tau \gg T$ [76].

DWS theory, which relates $\langle I\rangle$ to the electric field autocorrelation, $g_{1}(t)$ gives us 77

$$
V(T)=\frac{2}{T} \int_{0}^{T}(1-t / T)\left(g_{1}(t)\right)^{2} \mathrm{~d} t
$$

where $t$ is time. Taking Menon and Durian's expression for $g_{1}(t)$ in transmission [66] gives us the expression

$$
V(T)=\frac{1}{\beta}\left[\frac{\tau \sqrt{2 \pi}}{T} \operatorname{erf}\left(\frac{T}{\sqrt{2} \tau}\right)+\left(2 \exp \left(-\frac{T}{\sqrt{2} \tau}\right)^{2}\right)-2\left(\frac{\tau}{T}\right)^{2}\right]
$$

Since we know $T$ and we measure $1 / \beta$ in the sample at rest, the only unknown is $\tau$. Figure C8 shows some preliminary measurements of $V(T)$ for glass spheres in a water-fluidised bed. The camera used was a Phantom line-scan camera with with a frame rate of $10,000 \mathrm{~Hz}$ and an exposure time of $90 \mu \mathrm{s}$. Fitting the data with equation C.9 gives us the typical time scale of granular movement, $\tau$, shown in figure 4.4 .

\section{Photodiode sensor}

The sensors used in section 5.2.1 to measure $l^{*}$ in transmission are small selfbuilt circuits built around a HUV-1100BQ combined photodiode and operational amplifier. The calibration of the sensor is shown in figure D9: the sensor output signal as a function of laser input power (measured by a flux meter). The graph shows that the sensor has excellent linearity in the relevant intensity range. 


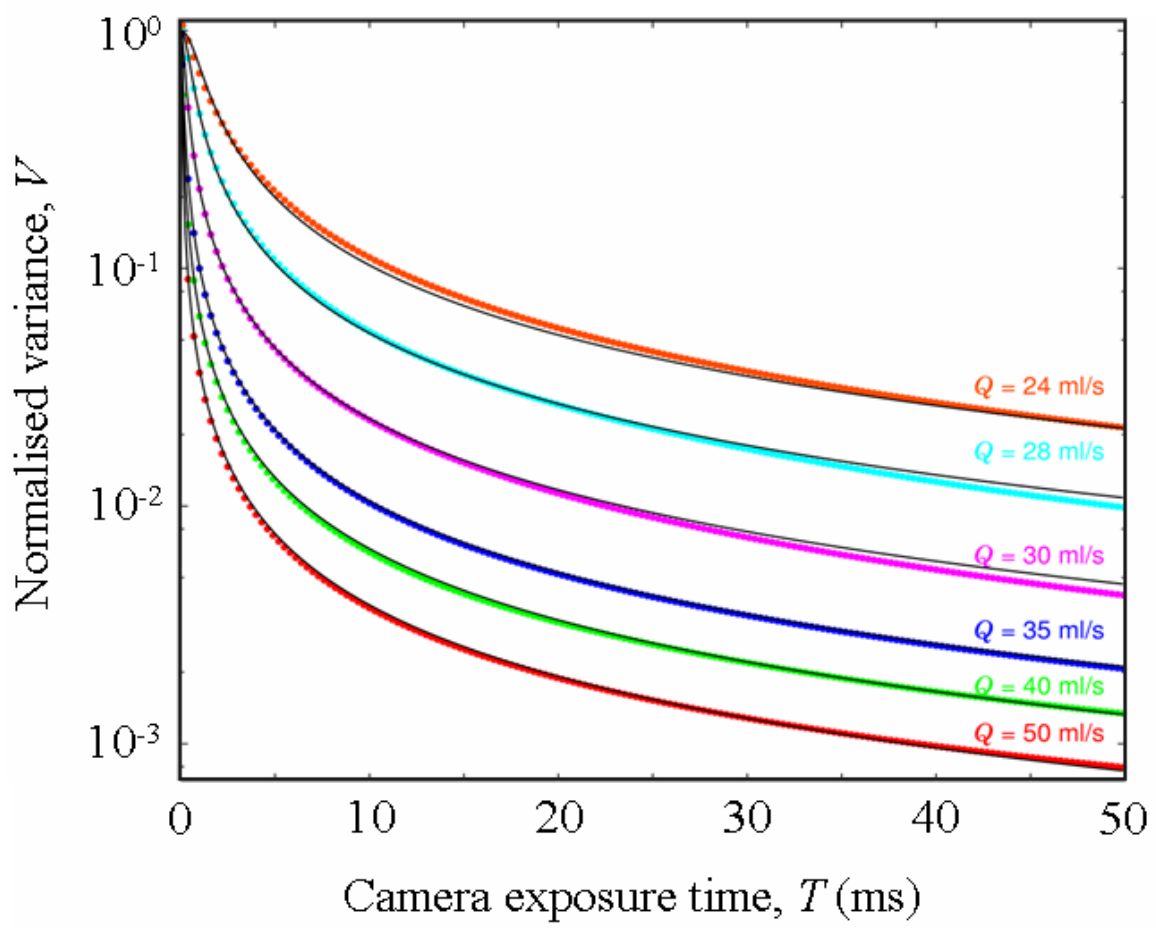

Figure C8.: $\quad$ Measurements of $V$ for a number of different fluidisation flow rates. Illumination was a Nd:YAG laser with $P=3 \mathrm{~W}$ and $\lambda=532 \mathrm{~nm}$. The camera was a Phantom camera with a frame rate of $10,000 \mathrm{~Hz}$ and an exposure time of $90 \mu \mathrm{s}$. Measured by Matthias Schröter. Lines are fits to equation C.9.

\section{E. Mie scattering}

In this section, we briefly present our predictions for the scattering functions of smooth soda-lime glass spheres in water. Figure E10 shows polar plots of the angular dependence of the scattered power predicted using the Bohren-HuffmanMie programmes of Mätzler [93, 94]. We take the refractive index of soda-lime glass $\eta=1.52$, radii $R$ appropriate to the grains we use in our experiments and light of wavelength $\lambda=532 \mathrm{~nm}$. The third plot is a for a polystyrene latex sphere such as is often used as a calibration in light scattering experiments. For the glass spheres, we observe a very strong anisotropy in the scattering power: almost all of the light passes through the grain with a scattering angle of less than $10^{\circ}$. The scattering function of the latex sphere is almost isotropic.

Table 8.1 shows the scattering asymmetry, $g \equiv\langle\cos \theta\rangle$ and the backscattering probability, $p_{b}$, calculated from Mie theory. $l^{*}$ is calculated at a volume fraction of 0.55 according to $l^{*}=(2 R) /(3 \phi(1-g))$. We use these quantities to calculate $l^{*}(\phi)$ (section 5.3) and to predict the backscattered spot in section 5.2.3. 


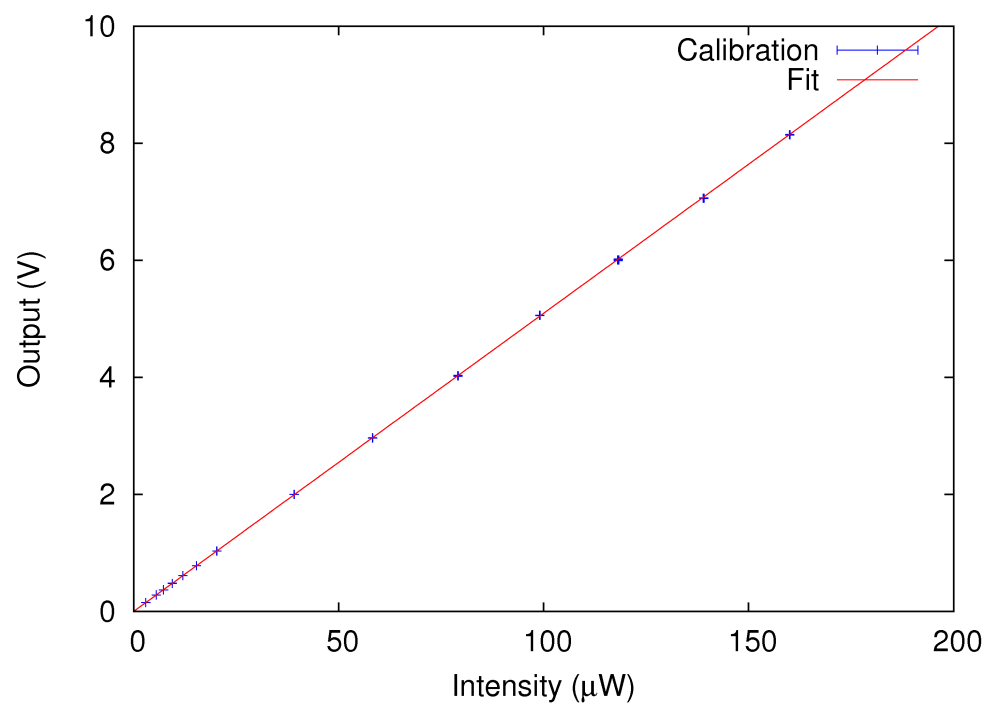

Figure D9.: Calibration of the photodiode sensor in the intensity range relevant to our experiment. Blue: measurement points. Red: a linear fit to the data giving the calibration factor.

\begin{tabular}{|c|c|c|}
\hline Observable & small grains & large grains \\
\hline$g$ & 0.9564 & 0.9067 \\
$p_{b}$ & 0.0349 & 0.1504 \\
$l^{\star}$ at $\phi=0.55$ & $13.9 d$ & $6.49 d$ \\
\hline
\end{tabular}

Table 8.1.: Predictions for Mie scattering of glass spheres made using the BohrenHuffman-Mie programmes of Mätzler 93, 94]. "Small" and "large" refer to the Mo-Sci grains of the sizes used in experiment. The reason for the non monotonic relationship between $l^{*}$ and particle radius in not yet clear to us. 


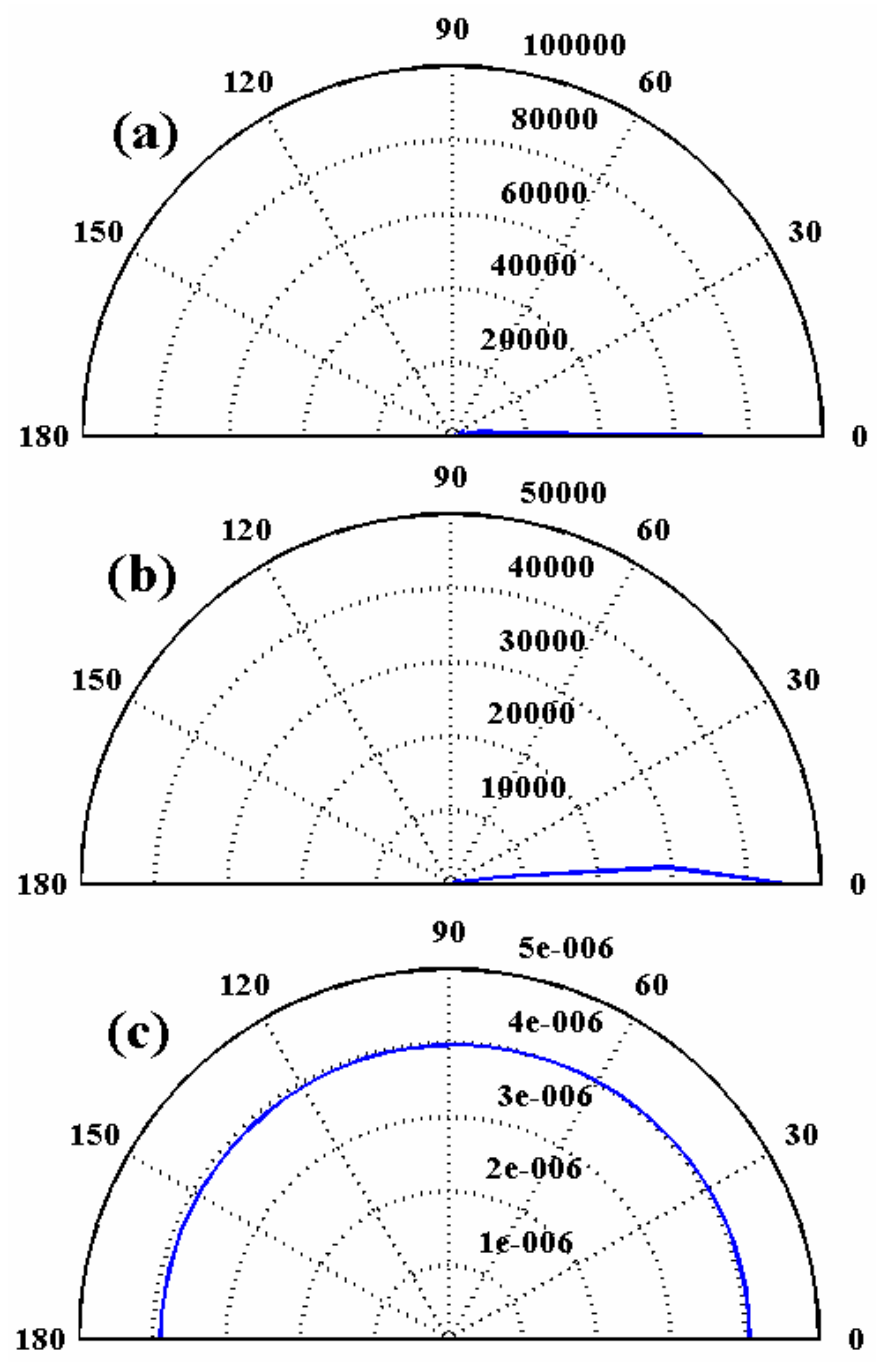

Figure E10.: $\quad$ Predicted angular dependence of the scattered power for (a) soda lime spheres of $R=70 \mu \mathrm{m}$ in water; (b) soda lime spheres of $R=115$ $\mu \mathrm{m}$ in water; and (c) latex spheres of $R=1 \mu \mathrm{m}$ in water. The scattering angle is in degrees: $0^{\circ}$ is the forward scattering direction and $180^{\circ}$ is backscattering. The radial axis gives the scattering power in arbitrary units. 



\section{Bibliography}

[1] M. A. Aguirre, J. G. Grande, A. Calvo, L. A. Pugnaloni, and J.-C. Géminard. Pressure Independence of Granular Flow through an Aperture. Physical Review Letters, 104(23):1-4, June 2010.

[2] A. Janda, I. Zuriguel, A. Garcimartín, L. A. Pugnaloni, and D. Maza. Jamming and critical outlet size in the discharge of a two-dimensional silo. Europhysics Letters, 84(4):44002, November 2008.

[3] G. H. L. Hagen. Über den Druck und die Bewegung des trockenen Sandes. Bericht über die zur Bekanntmachung geeigneten Verhandlungen der Königlich Preussischen Akademie der Wissenschaften zu Berlin., pages 35-42, 1852.

[4] B. P. Tighe and M. Sperl. Pressure and motion of dry sand: translation of Hagens paper from 1852. Granular Matter, 9(3-4):141-144, January 2007.

[5] H. A. Janssen. Versuche über Getreidedruck in Silozellen. Zeitschrift des Vereines deutscher Ingenieure, 39(35):1045-1048, 1895.

[6] M. Sperl. Experiments on corn pressure in silo cells translation and comment of Janssen's paper from 1895. Granular Matter, 8:59-65, December 2005 .

[7] W. A. Beverloo, H. A. Leniger, and J. Van de Velde. The flow of granular solids through orifices. Chemical engineering science, 15(3-4):260-269, 1961.

[8] M. E. Möbius. Clustering instability in a freely falling granular jet. Physical Review E, 74(5), November 2006.

[9] J. R. Royer, D. J. Evans, L. Oyarte, Q. Guo, E. Kapit, M. E. Möbius, S. R. Waitukaitis, and H. M. Jaeger. High-speed tracking of rupture and clustering in freely falling granular streams. Nature, 459(7250):1110-1113, June 2009.

[10] J. Duran. Sands, powders, and grains. Springer, New York, 2000.

[11] G. D. Scott, A. M. Charlesworth, and M. K. Mak. On the Random Packing of Spheres. The Journal of Chemical Physics, 40(2):611-612, 1964. 
[12] Anonymous. What is random packing? Nature, 239:488-489, 1972.

[13] M. Jerkins, M. Schröter, H. Swinney, T. Senden, M. Saadatfar, and T. Aste. Onset of Mechanical Stability in Random Packings of Frictional Spheres. Physical Review Letters, 101(018301), July 2008.

[14] G.R. Farrell, K. M. Martini, and N. Menon. Loose packings of frictional spheres. Soft Matter, 6(13):2925, 2010.

[15] M. Nicolas, P. Duru, and O. Pouliquen. Compaction of a granular material under cyclic shear. The European Physical Journal E, 3:309-314, 2000.

[16] L. Vanel, D. Howell, D. Clark, R. P. Behringer, and E. Clement. Memories in sand: experimental tests of construction history on stress distributions under sandpiles. Physical review. E, 60:R5040-3, November 1999.

[17] S. F. Edwards and R. B. S. Oakeshott. Theory of powders. Physica A, 157(3):1080-1090, 1989.

[18] J. T. Jenkins and M. W. Richman. Grad 's 13-Moment System for a Dense Gas of Inelastic Spheres. Archive for rational mechanics and analysis, 87(4):355-377, 1985.

[19] N. Brilliantov and T. Pöschel. Kinetic theory of granular gases. Oxford University Press, Oxford, 2004.

[20] L. da Vinci. da Vinci's notebooks. 1480.

[21] F. P. Bowden and D. Tabor. The Area of Contact between Stationary and between Moving Surfaces. Proceedings of the Royal Society of London. Series A, 169(938):391-413, 1939.

[22] G Amontons. De la résistance causée dans les machines (1). Histoire de l'Académie Royale des Sciences, 206, 1699.

[23] C. A. Coulomb. Essai sur une application des règles de maximis \& minimis à quelques problèmes de statique, relatifs à l'architecture. Académie des Sciences, Mémoires présentées par divers savants, 1776.

[24] L. Euler. Sur le frottement des corps solides. Mémoires de lacadémie des sciences de Berlin, 4:122-132, 1750.

[25] F. P. Bowden and D. Tabor. Mechanism of metallic friction. Nature, 150(3798):197-199, 1942.

[26] J. J. Bikerman. Surface Roughness and Sliding Friction. Reviews of modern physics, 16(1):53-68, 1944. 
[27] J. J. Bikerman. Effect of Surface Roughness on Rolling Friction. Journal of Applied Physics, 20(10), 1949.

[28] B. P. Tighe, J. H. Snoeijer, T. J. H. Vlugt, and M. van Hecke. The force network ensemble for granular packings. Soft Matter, 6(13):2908-2917, 2010 .

[29] S. Torquato, T. M. Truskett, and P. G. Debenedetti. Is random close packing of spheres well defined? Physical review letters, 84(10):2064-2067, March 2000.

[30] F. G. Bridges, A. Hatzes, and D. N. C. Lin. Structure, stability and evolution of Saturn's rings. Nature, 309:333-335, 1984.

[31] M. Montaine, M. Heckel, C. Kruelle, T. Schwager, and T. Pöschel. The Coefficient of Restitution as a Fluctuating Quantity. arXiv:1104.0049v1, 2011.

[32] N. V. Brilliantov, F. Spahn, J. M. Hertzsch, and T. Pöschel. Model for collisions in granular gases. Physical Review E, 53(5):5382-5392, May 1996.

[33] M. Huthmann and A. Zippelius. Dynamics of inelastically colliding rough spheres: Relaxation of translational and rotational energy. Physical Review E, 56(6):6275-6278, 1997.

[34] N. V. Brilliantov, T. Pöschel, W. T. Kranz, and A. Zippelius. Translations and Rotations Are Correlated in Granular Gases. Physical Review Letters, 98(12):128001, March 2007.

[35] W. T. Kranz, N. V. Brilliantov, T. Pöschel, and A. Zippelius. Correlation of spin and velocity in the homogeneous cooling state of a granular gas of rough particles. The European Physical Journal Special Topics, 179(1):91111, May 2010.

[36] T. Börzsönyi, T. Unger, and B. Szabó. Shear zone refraction and deflection in layered granular materials. Physical Review E, 80(060302(R)), December 2009.

[37] J. L. Anthony and C. Marone. Influence of particle characteristics on granular friction. Journal of Geophysical Research, 110(B08409), 2005.

[38] S. Ulrich, M. Schröter, and H. Swinney. Influence of friction on granular segregation. Physical Review E, 76(042301), October 2007.

[39] N. Pohlman, B. Severson, J. Ottino, and R. Lueptow. Surface roughness effects in granular matter: Influence on angle of repose and the absence of segregation. Physical Review E, 73(031304), March 2006. 
[40] T. Börzsönyi, T. Halsey, and R. Ecke. Two Scenarios for Avalanche Dynamics in Inclined Granular Layers. Physical Review Letters, 94(208001), May 2005.

[41] T. Börzsönyi and R. Ecke. Flow rule of dense granular flows down a rough incline. Physical Review E, 76(031301), September 2007.

[42] T. Börzsönyi, T. Halsey, and R. Ecke. Avalanche dynamics on a rough inclined plane. Physical Review E, 78(011306), July 2008.

[43] T. Halsey and A. Levine. How Sandcastles Fall. Physical Review Letters, 80(14):3141-3144, April 1998.

[44] M. B. Robertson. 5,792,368 (1998): Fluidized bed filter.

[45] S. G. Maas, G. Schaldach, E. M. Littringer, A. Mescher, U. J. Griesser, D. E. Braun, P. E. Walzel, and N. A. Urbanetz. The impact of spray drying outlet temperature on the particle morphology of mannitol. Powder Technology, 213:27-35, November 2011.

[46] D. Blair, N. Mueggenburg, A. Marshall, H. M. Jaeger, and S. R Nagel. Force distributions in three-dimensional granular assemblies: Effects of packing order and interparticle friction. Physical Review E, 63(041304):1-8, March 2001.

[47] G. A. C. M. Spierings. Wet chemical etching of silicate glasses in hydrofluoric acid based solutions. Journal of Materials Science, 28:6261-6273, December 1993.

[48] C.A.S. material safety data sheet No. 7664-39-3.

[49] S. H. Gimm and J. H. Kim. United States Patent 5,281,350.

[50] K. Shellenberger and B. E. Logan. Effect of molecular scale roughness of glass beads on colloidal and bacterial deposition. Environmental Science and Technology, 36(2):184-189, January 2002.

[51] G. K. Darbha, T. Schäfer, F. Heberling, A. Lüttge, and C. Fischer. Retention of latex colloids on calcite as a function of surface roughness and topography. Langmuir, 26(7):4743-4752, April 2010.

[52] P. Meakin. Fractals, scaling and growth far from equilibrium, volume 5. Cambridge University Press, Cambridge, 1998.

[53] I. Rodriguez-Iturbe and A. Rinaldo. Fractal river basins: chance and selforganization. Cambridge University Press, Cambridge, 2001. 
[54] I. N. Bronstein, K. A. Semendjajew, G. Musiol, and H. Mühlig. Taschenbuch der Mathematik. Harri Deutsch, Frankfurt am Main, 5 edition, 2000.

[55] A. Savage and J. Hyneman. Episode 57: Mentos and Soda, Mythbusters, Discovery Channel, first aired 9th August 2006, http://dsc.discovery.com/videos/mythbusters-diet-coke-and-mentos.html, 1996.

[56] T. S. Coffey. Diet Coke and Mentos: What is really behind this physical reaction? American Journal of Physics, 76(6):551-557, 2008.

[57] S. Courrech du Pont, P. Gondret, B. Perrin, and M. Rabaud. Granular Avalanches in Fluids. Physical Review Letters, 90(4), January 2003.

[58] S. Courrech du Pont, P. Gondret, B. Perrin, and M. Rabaud. Wall effects on granular heap stability. Europhysics Letters, 61(4):492-498, 2003.

[59] P. Jop, Y. Forterre, and O. Pouliquen. A constitutive law for dense granular flows. Nature, 441:727-730, June 2006.

[60] D. Schulze. Pulver und Schüttgüter: Fließeigenschaften und Handhabung. Springer, Heidelberg, 2006.

[61] A. Donev, I. Cisse, D. Sachs, E. A. Variano, F. H. Stillinger, R. Connelly, S. Torquato, and P. M. Chaikin. Improving the density of jammed disordered packings using ellipsoids. Science, 303(5660):990-993, March 2004.

[62] S. Utermann, P. Aurin, M. Benderoth, C. Fischer, and M. Schröter. Tailoring the frictional properties of granular media. Physical Review E, 84:031306, September 2011.

[63] I. Goldhirsch. Rapid Granular Flows. Annual Review of Fluid Mechanics, 35:267-293, January 2003.

[64] E. Ben-Naim and a. Zippelius. Singular Energy Distributions in Driven and Undriven Granular Media. Journal of Statistical Physics, 129(4):677-697, September 2007.

[65] D. I. Goldman and H. L. Swinney. Signatures of Glass Formation in a Fluidized Bed of Hard Spheres. Physical Review Letters, 96(14):145702, April 2006.

[66] N. Menon and D. J. Durian. Diffusing-Wave Spectroscopy of Dynamics in a Three-Dimensional Granular Flow. Science, 275(5308):1920-1922, March 1997. 
[67] R. L. Brown and J. C. Richards. Principles of powder mechanics. Pergamon press, Oxford, 1970.

[68] M. Schröter. Private communication, June 2007.

[69] P. C. Carman. Fluid flow through granular beds. Chemical Engineering Research and Design, 75:S32-S48, December 1997.

[70] R. Beetstra, M. A. van der Hoef, and J. A. M. Kuipers. Drag Force of Intermediate Reynolds Number Flow Past Mono- and Bidisperse Arrays of Spheres. AIChE Journal, 53(2):489-501, 2007.

[71] M. Nakagawa, S. A. Altobelli, A. Caprihan, E. Fukushima, and E. K. Jeong. Non-invasive measurements of granular flows by magnetic resonance imaging. Experiments in fluids, 16(1):54-60, 1993.

[72] S. Lasič, J. Stepišnik, A. Mohorič, I. Serša, and G. Planinšič. Autocorrelation spectra of an air-fluidized granular system measured by NMR. Europhysics Letters, 75(6):887-893, September 2006.

[73] R. D. Wildman, J. M. Huntley, J. P. Hansen, D. J. Parker, and D. A. Allen. Single-particle motion in three-dimensional vibrofluidized granular beds. Physical review. E, 62(3 Pt B):3826-3835, September 2000.

[74] W. Losert, D. G. W. Cooper, J. Delour, A. Kudrolli, and J. P. Gollub. Velocity statistics in excited granular media. Chaos, 9(3):682-690, September 1999.

[75] K. Nichol and K. E. Daniels. Equipartition of rotational and translational energy in a dense granular gas. arXiv:1111.2682; submitted to Physical Review E., 2011.

[76] P. Dixon and D. J. Durian. Speckle Visibility Spectroscopy and Variable Granular Fluidization. Physical Review Letters, 90(18), May 2003.

[77] G. Maret and P. E. Wolf. Static and dynamic multiple scattering of light. Science, 157:293-300, 1989.

[78] P. D. Kaplan, M. H. Kao, A. G. Yodh, and D. J. Pine. Geometric constraints for the design of diffusing-wave spectroscopy experiments. Applied optics, 32(21):3828-3836, 1993.

[79] J. Crassous. Diffusive wave spectroscopy of a random close packing of spheres. The European physical journal. E, 23:145-152, June 2007.

[80] P. D. Kaplan, A. D. Dinsmore, A. G. Yodh, and D. J. Pine. Diffusetransmission spectroscopy: a structural probe of opaque colloidal mixtures. Physical Review E, 50(6):4827-4835, 1994. 
[81] P. Lemieux, M. U. Vera, and D. J. Durian. Diffusing-light spectroscopies beyond the diffusion limit: The role of ballistic transport and anisotropic scattering. Physical Review E, 57(4):4498-4515, April 1998.

[82] C. M. Aegerter and G. Maret. Coherent backscattering and Anderson localization of light. Progress in Optics, 52, 2009.

[83] J. H. Li, A. A. Lisyansky, T. D. Cheung, D. Livdan, and A. Z. Genack. Transmission and Surface Intensity Profiles in Random Media. Europhysics Letters, 22(9):675-680, June 1993.

[84] W. Leutz and J. Ricka. On light propagation through glass bead packings. Optics Communications, 126:260-268, May 1996.

[85] M. U. Vera and D. J. Durian. Angular distribution of diffusely transmitted light. Physical Review E, 53(4):3215-3226, 1996.

[86] L. Bergmann and C. Schaefer. Lehrbuch der Experimentalphysik, Band 3: Optik. Walter de Gruyter, Berlin, 1987.

[87] D. J. Durian. Influence of boundary reflection and refraction on diffusive photon transport. Physical Review E, 50(2):857-866, 1994.

[88] E. Akkermans, P. E. Wolf, and R. Maynard. Coherent Backscattering of Light by Disordered Media: Analysis of the Peak Line Shape. Physical Review Letters, 56(14):1471-1474, December 1986.

[89] P. E. Wolf and G. Maret. Weak localization and coherent backscattering of photons in disordered media. Physical review letters, 55(24):2696-2699, 1985 .

[90] J. X. Zhu, D. J. Pine, and D. A. Weitz. Internal reflection of diffusive light in random media. Physical Review A, 44(6):3948, September 1991.

[91] S. Fiebig. Notizen zum Backscattering Setup. Private communication, 2008.

[92] P. Snabre and A. Arhaliass. Anisotropic scattering of light in random media: incoherent backscattered spotlight. Applied optics, 37(18):4017-4026, June 1998.

[93] C. F Bohren and D. R. Huffman. Absorption and scattering of light by small particles. Wiley, New Zork, 1983.

[94] C. Mätzler. MATLAB Functions for Mie Scattering and Absorption. Technical report, Microwave department, Institute for Applied Physics, University of Bern, 2002. 
[95] P. Snabre and J. Crassous. Multispeckle diffusing wave spectroscopy of colloidal particles suspended in a random packing of glass spheres. The European physical journal. E, 29(2):149-155, June 2009.

[96] A. Abate and D. J. Durian. Effective Temperatures and Activated Dynamics for a Two-Dimensional Air-Driven Granular System on Two Approaches to Jamming. Physical Review Letters, 101(24), December 2008.

[97] M. Scheel, R. Seemann, M. Brinkmann, M. Di Michiel, A. Sheppard, B. Breidenbach, and S. Herminghaus. Morphological clues to wet granular pile stability. Nature materials, 7:189-193, March 2008.

[98] D. J. Hornbaker, R. Albert, I. Albert, A. L. Barabási, and P. Schiffer. What keeps sandcastles standing? Nature, 387:765, 1997.

[99] T. G. Mason, A. J. Levine, D. Erta, and T. C. Halsey. Critical angle of wet sandpiles. Physical review. E, 60(5):R5044-R5047, November 1999.

[100] A. Einstein. Über die von der molekularkinetischen Theorie der Wärme geforderte Bewegung von in ruhenden Flüssigkeiten suspendierten Teilchen. Annalen der Physik, 17(8):639-341, 1905.

[101] C. Song, P. Wang, and H. A. Makse. Experimental measurement of an effective temperature for jammed granular materials. Proceedings of the National Academy of Sciences, 102(7), 2005.

[102] G. D'Anna, P. Mayor, A. Barrat, V. Loreto, and F. Nori. Observing brownian motion in vibration-fluidized granular matter. Nature, 424(6951):909912, August 2003.

[103] A. Buck. The effective granular temperature based on the fluctuationdissipation theorem. PhD thesis, University of Texas (Austin), 2007.

[104] P. Wang, C. Song, Y. Jin, and H. A. Makse. Jamming II: Edwards statistical mechanics of random packings of hard spheres. Physica A, 390:427-455, February 2011.

[105] S. Mukhopadhyay and J. Peixinho. Packings of deformable spheres. Physical Review E, 84(1):2-6, July 2011.

[106] G. E. Schröder-Turk, W. Mickel, M. Schröter, G. W. Delaney, M. Saadatfar, T. J. Senden, K. Mecke, and T. Aste. Disordered spherical bead packs are anisotropic. Europhysics Letters, 90(3), May 2010.

[107] J.-F. Métayer, P. Richard, A. Faisant, and R. Delannay. Electrically induced tunable cohesion in granular systems. Journal of Statistical Mechanics, (8):P08003, August 2010. 


\section{Acknowlegements}

I would like to thank the following people for their help.

Matthias Schröter, for his supervision and for believing I could make it;

My examining committee: Stephan Herminghaus, Sarah Köster, Stefan Luther, Holger Nobach, Christoph Schmidt and Annette Zippelius;

My kind proofreaders Karen Daniels, Annika Döring, Christoph Gögelein, John May, Jean-François Métayer, Chih-Wei Peng and Andre Utermann;

My office mates and SMGM colleagues, who have always been ready to help: Philipp Aurin, Karen Daniels, Annika Döring, Christoph Gögelein, Jean-François Métayer, Julie Murison, Max Neudecker, Semra Ötztürk-Mert, Welm Pätzold, Chih-Wei Peng, Sravanti Uppaluri and SongChuan Zhao;

My brilliant students: Philipp Aurin, Jonathan Kohler and Dominik Michler;

My collaborators: Susanne Fiebig and Cornelius Fischer;

Additionally: Christof Aegerter, Silke Allekamp, Ilenia Battiato, Markus Benderoth, Alexander Buck, Steve Clappison, Jann Ohle Claussen, Thomas Eggers, Mattieu Gibert, Kristian Hantke, Kier Heeck, Harald Heinrici, Joachim Hesse, Dirk Huch, Christian Jacobs, Wolf Keiderling, Konstantina Kostourou, Udo Krafft, Bernhard Krey, Ortwin Kurre, Corinna Maaß, Georg Maret, Jan Rector, Frank Rietz, Karin Sand, Udo Schminke, Sabine Schlusselburg, Anupam Sengupta, Eric Stellamans, Zvonko Tadič, Monika Teuteberg, Jürgen Vollmer and Birgit Will;

And my family: Angelika Dennis, Michael Dennis, Rachel Dennis, Jenny May, John May and Andre Utermann. 



\section{Publications}

S. Utermann, P. Aurin, M. Benderoth, C. Fischer and M Schröter Tailoring the frictional properties of granular media

Phys. Rev. E 84, 031306 (2011)

J. P. Embs, S. May, C. Wagner, A. Kityk, A. Leschhorn and M. LüCKE Measuring the transverse magnetization of rotating ferrofluids Phys. Rev. E 73, 036302 (2006) 

$>$ Griff

$$
\begin{aligned}
& \text { toen Griff rijn } \\
& \text { scriptic eindeligk } \\
& \text { of was, bleck de } \\
& \text { menselyke soort } \\
& \text { inmidblels alween } \\
& \text { iitgestorven. }
\end{aligned}
$$

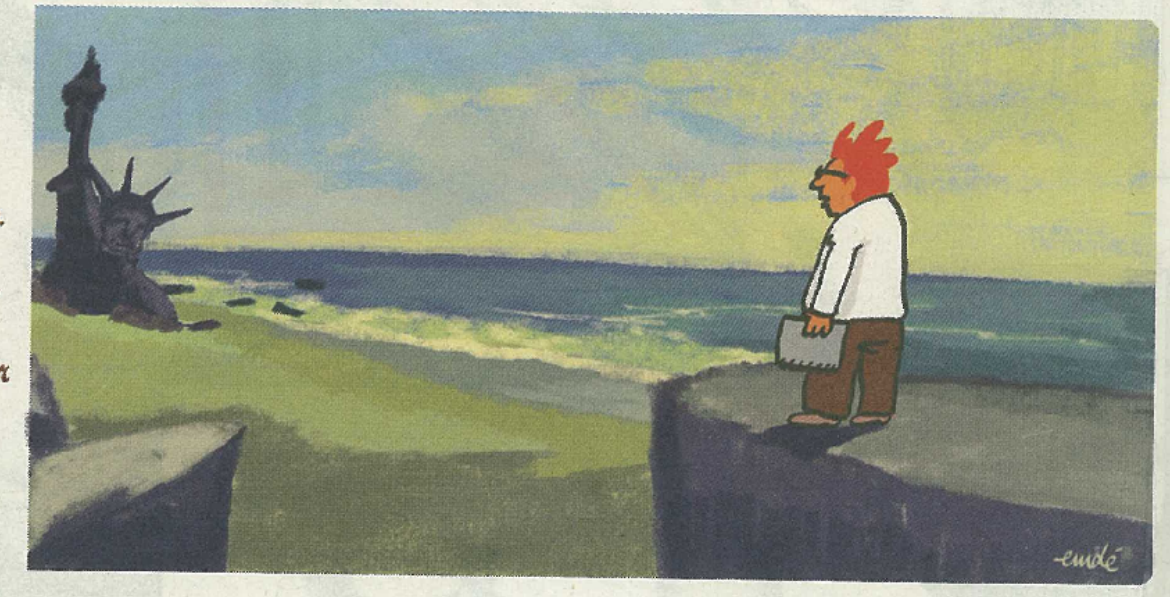

By the time Griff had finished his thesis, it appeared that humankind had died out.

Ad Valvas (2007); reproduced with kind permission of Merlijn Draisma. 